Florida International University FIU Digital Commons

$11-12-2014$

\title{
Assessing Organizational Image: Triangulation Across Different Applicant Perceptions, Website, and Facebook Features
}

\author{
Alejandra Matamala \\ Florida International University, amata005@fiu.edu
}

DOI: $10.25148 /$ etd.FI14110760

Follow this and additional works at: https://digitalcommons.fiu.edu/etd

Part of the Industrial and Organizational Psychology Commons

\section{Recommended Citation}

Matamala, Alejandra, "Assessing Organizational Image: Triangulation Across Different Applicant Perceptions, Website, and Facebook Features" (2014). FIU Electronic Theses and Dissertations. 1740.

https://digitalcommons.fiu.edu/etd/1740 


\title{
FLORIDA INTERNATIONAL UNIVERSITY
}

Miami, Florida

\section{ASSESSING ORGANIZATIONAL IMAGE: TRIANGULATION ACROSS \\ DIFFERENT APPLICANT PERCEPTIONS, WEBSITE, AND FACEBOOK FEATURES}

\author{
A dissertation submitted in partial fulfillment of the \\ requirements of the degree of \\ DOCTOR OF PHILOSOPHY
}

in

PSYCHOLOGY

by

Alejandra C. Matamala

2014 
To: Interim Dean Michael R. Heithaus

College of Arts and Sciences

This dissertation, written by Alejandra C. Matamala, and entitled Assessing Organizational Image: Triangulation Across Different Applicant Perceptions, Website, and Facebook Features, having been approved in respect to style and intellectual content, is referred to you for judgment.

We have read this dissertation and recommend that it be approved.

Valentina Bruk-Lee

Paulette Johnson

Jesse Michel

Chockalingam Viswesvaran, Major Professor

Date of Defense: November 12, 2014

The dissertation of Alejandra C. Matamala is approved.

\begin{tabular}{r}
$\begin{array}{r}\text { Interim Dean Michael R. Heithaus } \\
\text { College of Arts and Sciences }\end{array}$ \\
\hline Dean Lakshmi N. Reddi \\
University Graduate School
\end{tabular}

Florida International University, 2014 


\section{DEDICATION}

I dedicate this dissertation to my mom and my sisters. I thank them for their

encouragement, love, and support, without which the completion of this work would not have been possible. 


\section{ACKNOWLEDGMENTS}

I would like to thank my committee members for their assistance, support, and advice. I would especially like to thank my major professor, Chockalingam

Viswesvaran, whose guidance enabled me to accomplish this study successfully. Also, a special thanks to Vicky Pace for her contributions to the early stages of this study.

Lastly, I am indebted to my colleagues and friends who offered unconditional support throughout the process. 


\section{ABSTRACT OF THE DISSERTATION \\ ASSESSING ORGANIZATIONAL IMAGE: TRIANGULATION ACROSS \\ DIFFERENT APPLICANT PERCEPTIONS, WEBSITE, AND FACEBOOK}

$$
\text { FEATURES }
$$

by

Alejandra C. Matamala

Florida International University, 2014

Miami, Florida

Professor Chockalingam Viswesvaran, Major Professor

This study examined the role of corporate websites and company Facebook profiles in shaping perceptions of organizational image in the recruitment context. A primary purpose of this research was to determine whether or not perceptions of organizational image vary across different web-based recruitment methods, specifically examining corporate websites and social networking (SNW) sites, such as company Facebook profiles. A secondary goal was to determine how these perceptions of image are shaped by the objective components of websites and Facebook profiles. Finally, this study sought to determine the most influential components of websites and Facebook profiles, in terms of impacting image, to better understand how organizations can maximize their web-based recruitment efforts.

A total of 102 companies selected from Fortune Magazine's 2011 top 500 were chosen for the study. Perceptions of organizational personality as well as objective assessments of personality were gathered for each organization in a two phase approach. Results indicate that exposure to corporate websites and company Facebook profiles do 
influence perceptions of image in different ways. Furthermore, individual components of the websites were identified as key drivers for influencing specific image dimensions, particularly for company Facebook pages. Findings are beneficial for advising practitioners on how to best manage their web-based recruitment sources in order to maximize efficiency. The present study serves to further our understanding of the process through which perceptions of organizational image are influenced by new recruitment sources. 


\section{TABLE OF CONTENTS}

CHAPTER

PAGE

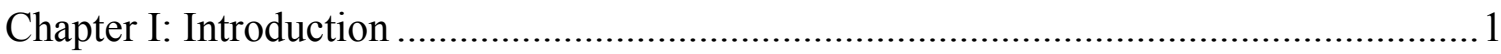

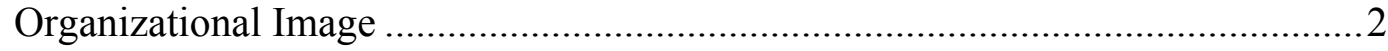

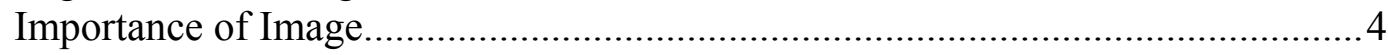

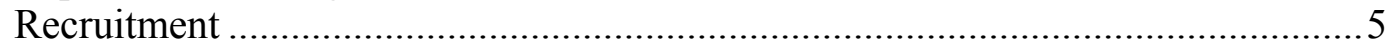

Image and Recruitment.......................................................................

Person-Organization Fit..........................................................................

Organizational Attraction ....................................................................

Image and Recruitment Sources ................................................................... 10

Image and Corporate Websites.................................................................12

Image and Facebook ............................................................................. 14

Differences in Image across Web-based Recruitment Media .................................. 16

Website Indicators of Organizational Image .......................................................... 17

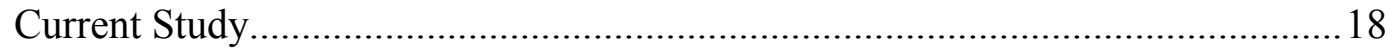

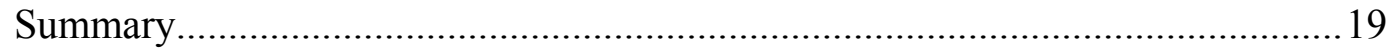

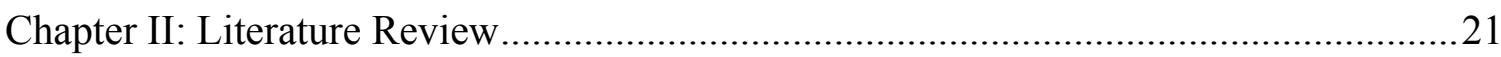

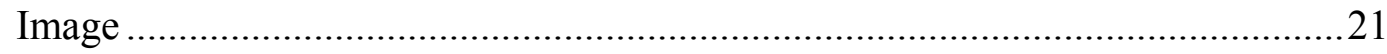

Internally-Focused Conceptualizations of Image .......................................23

Construed External Image. ..........................................................23

Projected Image .....................................................................24

Desired Future Image. ................................................................2.

Externally-Focused Conceptualizations of Image ......................................25

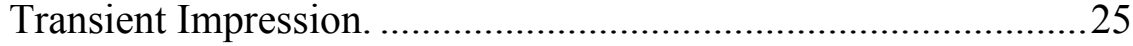

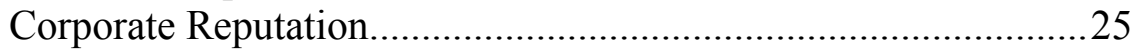

Image Conceptualizations in the Business Literature …………………....26

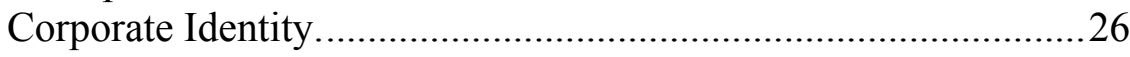

Employer Branding. ...................................................................2

Unifying Conceptualizations of Image .....................................................27

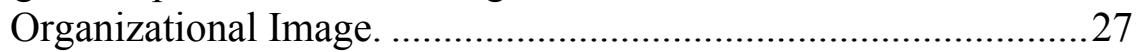

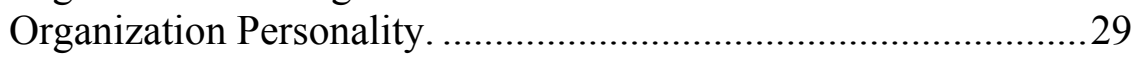

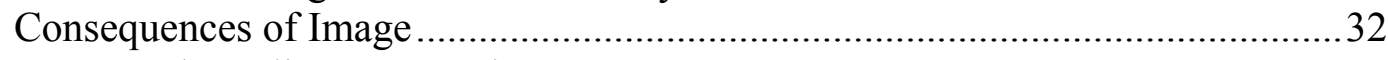

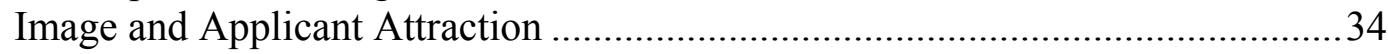

Person-Organization Fit and Attraction....................................................35

Image Congruity Theory and Attraction ...................................................38

Self-Continuity and Attraction ................................................................39

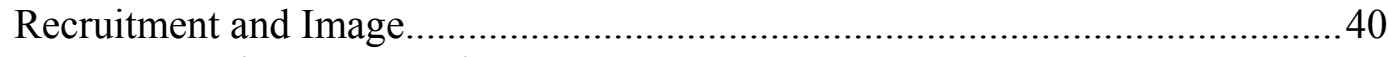

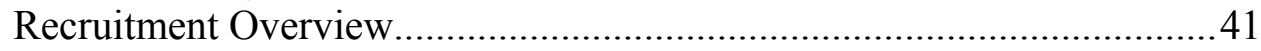

Web-Based Recruitment......................................................................42

Web-Based Recruitment Methods.............................................................43

Webpage Design and Perceptions of Attraction...........................................47 


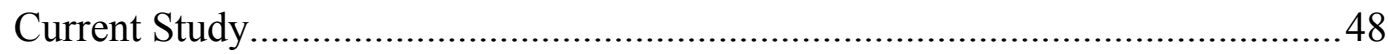

Website Design and Perceptions of Image .................................................50

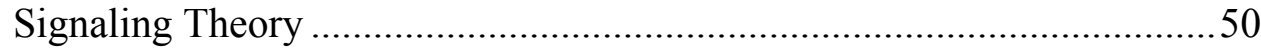

Websites and Perceptions of Organizational Culture.................................51

Websites and Perceptions of Individual Personality ……………………...53

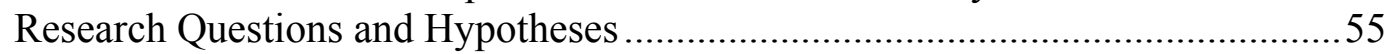

Identifying Objective Indicators..............................................................55

Congruence between Subjective and Objective Image ..............................62

Divergence across Sources of Image.............................................................63

Relative Predictive Validity of Objective Indicators....................................64

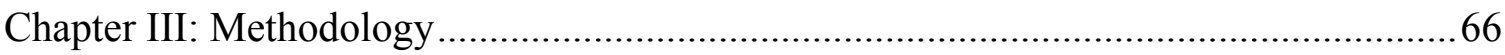

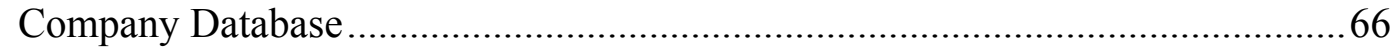

Phase One: Perceptions of Organizational Personality ............................................67

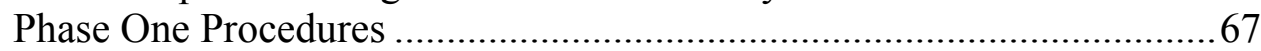

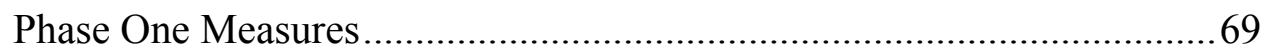

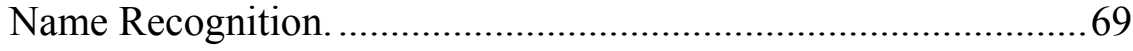

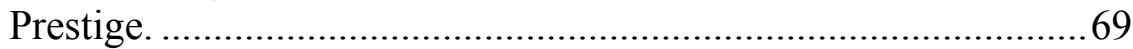

Self-Report Organizational Personality..........................................70

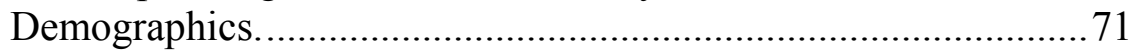

Test Items. ..................................................................................

Phase Two: Developing Objective Ratings of Organizational Personality ............71

Phase Two Procedures............................................................................

Step One: Indicator Ratings...........................................................72

Step Two: Content Validation.....................................................73

Step Three: Empirical Validation..................................................74

Measures for Phase Two ..................................................................

Website Indicators of Organizational Personality.........................75

Facebook Profile Indicators of Organizational Personality............77

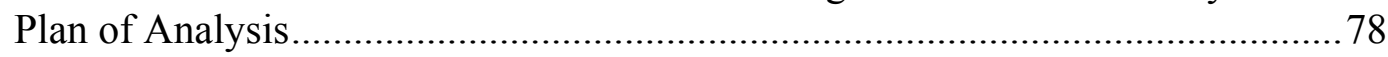

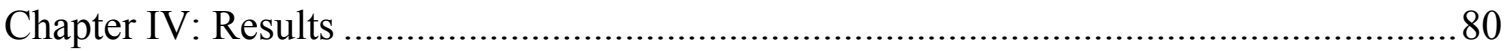

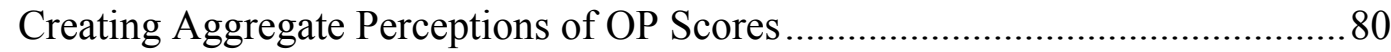

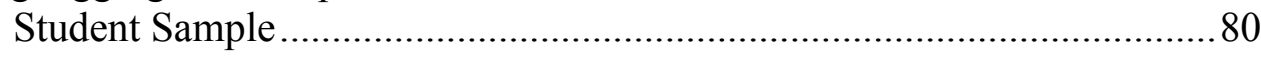

Preliminary Confirmatory Factor Analyses...............................................81

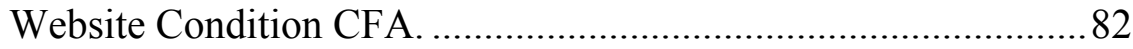

Facebook Condition CFA............................................................... 83

No Exposure Condition CFA. …………………………….............. 84

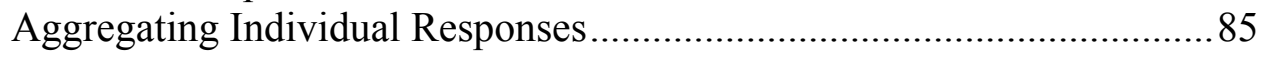

Research Questions 1-5: Development of Objective Indicators for OP .................88

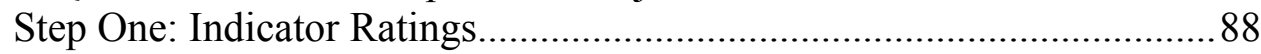

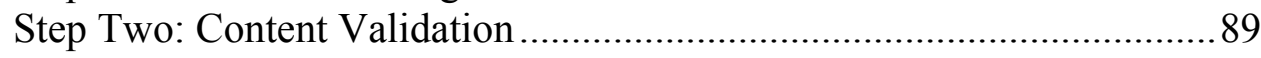

Step Three: Empirical Validation ............................................................99

Website Indicator CFA ……………………………..................... 
Facebook Indicator CFA.

Hypothesis 1 and 2: Testing Convergence between Self-Report and Objective Scores

Hypothesis 1: Website Condition Convergence........................................96

Supplementary Analysis for Hypothesis 1 .........................................97

Hypothesis 2: Facebook Condition Convergence ................................. 98

Supplementary Analysis for Hypothesis 2 ........................................... 99

Hypothesis 3 and 4: Source Differentiation Analyses across Recruitment

Media

Hypothesis 3: Perceptions of OP Source Differentiation.

Supplementary Analyses for Hypotheses 3

Hypothesis 4: Objective OP Scores Source Differentiation....................105

Objective Indicator Predictive Validity 106

Research Question 6: Website Indicator Predictive Validity

Regressions.

Research Question 7: Facebook Indicator Predictive Validity

Regressions

Chapter V. Discussion

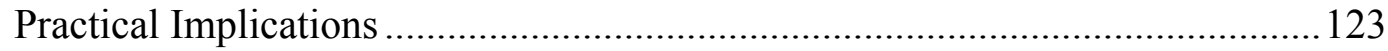

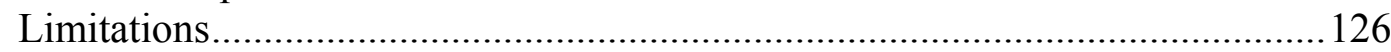

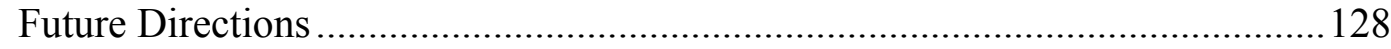

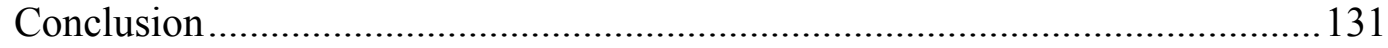

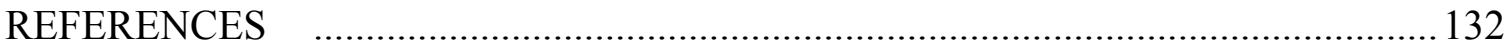

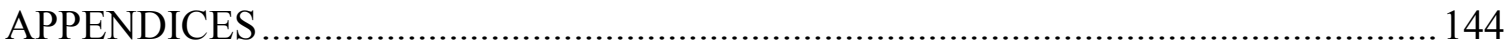

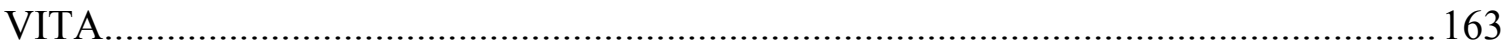




\section{LIST OF TABLES}

TABLE

PAGE

Table 1. Comparison of Alternative Confirmatory Factor Analysis Models for Perceptions of Organizational Personality 85

Table 2. Means, Standard Deviations, and Correlations of Perceptions of Organizational Personality for the Website Condition

Table 3. Means, Standard Deviations, and Correlations of Perceptions of Organizational Personality for the Facebook Condition $(\mathrm{N}=102)$.... 87

Table 4. Means, Standard Deviations, and Correlations of Perceptions of Organizational Personality for the No Exposure Condition $(\mathrm{N}=50) \ldots \ldots \ldots \ldots \ldots . . . . .88$

Table 5. Summary of Sort Task Results for Website Indicators....................................... 90

Table 6. Summary of Sort Task Results for Facebook Indicators ................................... 90

Table 7. Comparison of Alternative Confirmatory Factor Analysis Models for Objective Indicators of Organizational Personality 93

Table 8. Summary of Convergent Analysis for the Website Condition ( $\mathrm{N}=102)$ 97

Table 9. Summary of Supplementary Convergent Analysis for the Website Condition $(\mathrm{N}=102)$. 98

Table 10. Summary of Convergent Analysis for the Facebook Condition $(\mathrm{N}=102)$..... 99

Table 11. Summary of Supplementary Convergent Analysis for the Facebook Condition $(\mathrm{N}=102)$. 100

Table 12. Means and Standard Deviations of OP dimensions across the Three Recruitment Methods $(\mathrm{N}=50)$

Table 13. Means and Standard Deviations of OP dimensions across the Two Recruitment Methods $(\mathrm{N}=102)$.

Table 14. Multiple Linear Regression for Objective Website Indicators Predicting Perceptions of Boy Scout $(\mathrm{N}=102)$. 108

Table 15. Multiple Linear Regression for Objective Website Indicators Predicting Perceptions of Innovation $(\mathrm{N}=102)$ 108 
Table 16. Multiple Linear Regression for Objective Website Indicators Predicting

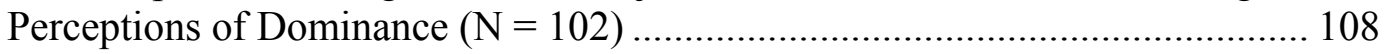

Table 17 Multiple Linear Regression for Objective Website Indicators Predicting

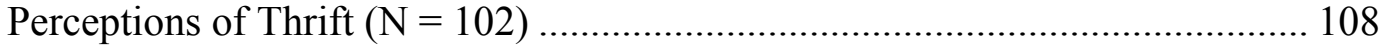

Table 18. Multiple Linear Regression for Objective Website Indicators Predicting

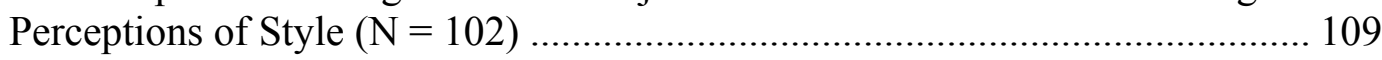

Table 19. Multiple Linear Regression for Objective Facebook Indicators Predicting

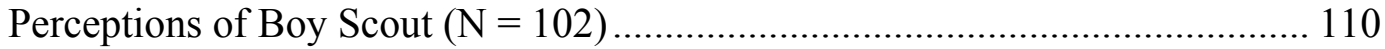

Table 20. Multiple Linear Regression for Objective Facebook Indicators Predicting

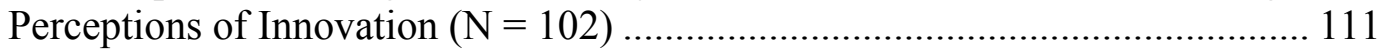

Table 21. Multiple Linear Regression for Objective Facebook Indicators Predicting

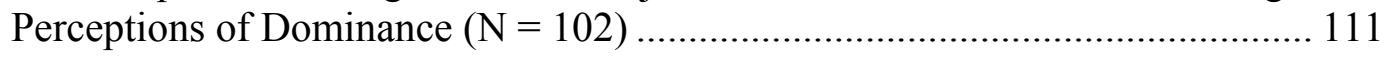

Table 22. Multiple Linear Regression for Objective Facebook Indicators Predicting

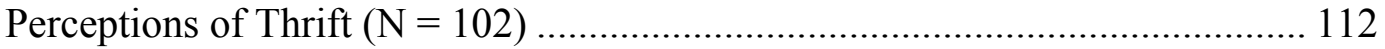

Table 23. Multiple Linear Regression for Objective Facebook Indicators Predicting

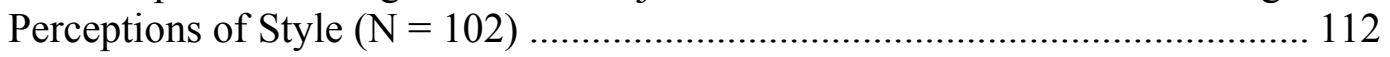

Table 24. Key Objective Components Related to Image for Website and Facebook..... 125 


\section{Chapter I: Introduction}

Organizational image has long served as a way for organizations to communicate their core values and goals to internal and external members. Although organizational image impacts many different business processes, it plays a pivotal role in the recruitment phase of employee selection systems. It is important for organizations to relay information to applicants during the recruitment phase that will aid them in their job search process. An organization's image conveys information about its values, culture, and goals, which in turn allows the job seeker to become more familiar with the personality of the organization. Recent technological advances have impacted the manner through which organizations can communicate their image to external members. Companies are now able to reach potential applicants through web-based recruitment platforms faster and easier than they ever have before. It is vital that organizations understand the intricacies of new web-based recruitment methods, as compared to traditional recruitment methods, when it comes to communicating information with possible job applicants. Unfortunately, limited research exists regarding how potential applicants form perceptions of image from web-based recruitment media (e.g., Braddy, Meade, \& Kroustalis, 2006; Braddy, Meade, Michael, \& Fleenor, 2009).

A primary purpose of the present research is to determine whether or not perceptions of organizational image vary across different web-based recruitment methods, specifically examining corporate websites and social networking (SNW) sites, such as company Facebook profiles. For example, do job seekers perceive an organization as having the same organizational image whether or not they are exposed to 
web-based recruitment materials? More so, do perceptions of image vary depending on exposure to corporate websites of company Facebook profiles? A secondary goal of the research is to determine how these perceptions of image are shaped by the objective components of websites and Facebook profiles. In other words, how do the objective features and content on the webpages influence perceptions of organizational image? A final goal of the study is to determine the most influential components of websites and Facebook profiles, in terms of impacting image, to better understand how organizations can maximize their web-based recruitment efforts.

\section{Organizational Image}

According to Dutton, Dukerich, and Harquail (1994), organizational image can be described through two broad concepts: (1) an organization's image is partly determined by what members of the organization believe are "distinctive, central, and enduring" characteristics of the organization; (2) image is also partly dependent on members' views concerning how outsiders think about the company (Allen, Mahton, \& Otondo, 2007; Billsberry, 2007; Gioia, Shultz, \& Corley, 2000; Lievens, Van Hoye, \& Anseel, 2007). For the purpose of recruitment, the latter conceptualization is of particular interest since this reflects the importance of image perceptions from the perspective of job seekers.

In line with Dutton and colleagues' second conceptualization, Berg (1985) defined organizational image as the public's perception of an organization that is often linked to a given action or event. Similarly, Frombrun (1996) described organizational reputation as the collective evaluation (by non-members) of an organization's actions and accomplishments. It is important to highlight that Frombrun's conceptualization implies 
a more global, broad appraisal of image compared to that of Berg's. Recent research by Lievens (2006) supports Frombrun's definition and views organizational image as people's global impression of an organization, derived from loose structures of knowledge and beliefs of an organization. Lievens (2006) argues for two general components of organization image stemming from brand equity theory: (1) instrumental attributes represent a group of objective attributes that people associate with an organization (e.g., organization policies); and (2) symbolic attributes are comprised of trait-related inferences concerning an organization (e.g., intangible perceptions) (Aaker, 1997). Furthermore, scholars believe that symbolic attributes serve as a way to describe organizations in terms of personality traits (e.g., honest, prestigious, etc.) (Lievens \& Highhouse, 2003).

Also stemming from Aaker's brand equity theory, Slaughter, Zickar, Highhous, and Mohr (2004) developed the construct of organizational personality, defined as the "set of human personality characteristics perceived to be associated with an organization (p.86)." The five dimensional construct consists of five personality dimensions: Boy Scout, Innovation, Dominance, Thrift, and Style. The first dimension, Boy Scout, represents an organization's honesty, helpfulness, family-orientation, and attentiveness to people. Innovation refers to how exciting, unique, or creative an organization is. The Dominance factor relates to the extent to which an organization is considered successful, popular, or active. Thrift relates to an organization being viewed as low budget, simple, or sloppy. Lastly, Style encompasses organizational characteristics seen as stylish, trendy, and contemporary (Slaughter et al., 2004). Researchers assert that outsiders are able to make an assessment of an organization's personality even when dealing with 
limited exposure to the organization (Lievens \& Highhouse, 2003; Slaughter et al., 2004). Based on research findings, potential applicants would be able to form perceptions of organizational personality from limited exposure to recruitment material. Furthermore, these perceptions of personality are shown to influence applicant attraction, job pursuit intentions, and reputation perceptions (Slaughter et al., 2004).

\section{Importance of Image}

In order for an organization to successfully market themselves to a target audience, they must be able to distinguish themselves from competitors. A key way organizations can differentiate themselves is through their organizational image (Aaker, 1997; Cable \& Turban, 2003; Lievens, 2006; Siguaw, Mattila, \& Austin, 1999; Scott \& Lane, 2000). Image can have lasting impressions on a number of different stakeholders, such as internal employers, investors, customers and clients, and prospective job applicants. Although the business processes that image impacts depend on the role of the stakeholder, image has consistently been linked to significant organizational outcomes.

In the context of employee selection, an organization's image impacts job seekers' feelings and attraction towards the organization as a desirable place to work. According to marketing research, organizational image, or brands, can profoundly influence the attraction of job applicants to organizations (Allen et al., 2007). More so, image's impact on perceptions is most prevalent in the early phases of recruitment when the applicant's possess limited knowledge of the job or organization. Since the early recruitment phase is instrumental in building the applicant pool for future selection steps, 
perceptions of image can have lasting effects on the duration of the selection process (Lievens, 2006).

The impact of organizational image in the recruitment process has become even more salient than before as a consequence of a growing shift towards web-based recruitment methods (e.g., Lloren \& Kellough, 2007; Maurer \& Liu, 2007). These recent technological advancements have made the need for differentiation more prominent because of the widespread reach and immediate impact offered by web-based recruitment media. Organizational websites, for example, are becoming an increasingly popular recruitment method (Allen et al., 2007; Cappelli, 2001). Websites offer reduced costs and can reach a large number of potential applicants quicker, compared to traditional recruitment methods (Rynes \& Cable, 2003). More so, compared to traditional recruitment media, websites provide a richer and more appropriate medium, through which organizations can promote their core image (Chapman \& Webster, 2003). Given their pressing popularity and increased benefits, it is essential that scholars better understand how to project image efficiently through web-based recruitment methods.

The following sections provides a more detailed examination of current trends in recruitment, as well as how these new trends impact organizational image and subsequent applicant perceptions.

\section{Recruitment}

Despite the many changes and advancements over the decades, companies still face many of the same hurdles in terms of attracting high quality applicants. Statistics indicate that the average U.S. company spends between $\$ 1,000$ and $\$ 8,000$ on 
recruitment costs per applicant (Greenburg, 1998). With proper planning, this cost can prove to be highly beneficial for organizations in terms helping them gain, and maintain, a competitive advantage in the war for talent.

Barber (1998) defines recruitment as "those practices and activities carried on by an organization with the primary purpose of identifying and attracting potential employees" (Barber, 1998, p.5). More specifically, a primary goal of recruitment is to attract future employees by actively reaching out and inviting applicants to become an organizational member (Barber, 1998; Breaugh \& Starke, 2000). Barber (1998) outlines three phases to the recruitment process: generating applicants, maintaining applicant interest in the organization, and influencing job choice. The present study focuses on applicant attraction in the earliest stages of the generating applicants phase. It is essential to note that the attraction phase is particularly crucial for determining the success of the subsequent recruitment phases since this is when the initial pool of applicants is shaped (Allen et al., 2007).

Recruitment activities involve many activities, such as defining the target population, deciding the type of advertisement to be used, and determining the source and content of the recruitment tool. Ultimately, these activities should be used as a way to persuade job seekers to pursue employment with the organization (Barber, 1998). As such, recruitment serves as the first stage of a selection system and allows companies to effectively target individuals appropriate for specific needs and goals (Cooper, Robertson, \& Tinline, 2003). These targeted recruitment efforts allow companies to eliminate applicants that would not be a good fit for the organization early on in the 
selection process. Without this crucial step, time and resources could be spent hiring and training applicants that ultimately would not remain with the organization for long. The selection-focused approach, which entails investing money in planning and recruitment, is more cost effective than spending money on turnover costs (Cascio \& Aguines, 2005).

\section{Image and Recruitment}

Organizational image is arguable one of the most influential factors in the early phases of employee recruitment. Perceptions of image have been consistently link to both applicant attraction and subsequent job-choice decision making (e.g., Lievens, 2007; Lievens \& Highhouse, 2003; Uggurslev, Fassina, \& Kraichy, 2012). More specifically, research has found that higher levels of person-organization fit are associated with greater attraction to organizations (e.g., Chapman, Uggerslev, Carroll, \& Jones, 2005; Judge \& Cable, 1996, 1997; Turban \& Keon, 1993) as well as higher job acceptance intentions (Bretz \& Judge, 1994; Judge \& Bretz, 1992), compared to individuals with lower personorganization fit. We now examine one such mechanism through which image leads to increased attraction, followed by a detailed look at the image-attraction link.

\section{Person-Organization Fit}

One of the most common things that potential applicants assess during the recruitment phase is the extent to which they "fit" with an organization, or personorganization (P-O) fit. Kristof (1996) defines P-O fit as the "compatibility between people and the organization in which they work" (Kristof, 1996, p.1). Studies by Schneider and other researchers suggest that $\mathrm{P}-\mathrm{O}$ fit represents the similarities, or congruence, between organizational work values and those of the organization's 
employees (Chatman \& Jehn, 1994; Kristof, 1996; Schneider, Goldstein, \& Smith, 1995).

Similarly, it can be viewed as the goal congruence between the organization and the individual.

According to theory, both through the Attraction-Selection-Attrition (ASA) framework (Byrne, 1971) and the Social Identity perceptions (Tajfel \& Turner, 1985), individuals are attracted to and seek employment with organizations that exhibit characteristics similar to their own (Schneider et al., 2006). The ASA framework suggests that individuals prefer to work for an organization whose attributes align with their personal characteristics (Cable \& Judge, 1996; Kristof, 1996; Schneider, 1987). Similarly, the social identity theory proposes that individuals seek to join organizations in an attempt to strengthen their self-concepts. According to research on social psychology, an organization's values are reflected into those who work there (Dutton \& Dukerich, 1991), in a sense linking the organization to the individual's social identity (Ashforth \& Mael, 1989). Consequently, organizations that are similar to the job seeker will in turn be more attractive and satisfying (e.g., Cable \& Judge, 1986; Kristof-Brown, Zimmerman, \& Johnson, 2005).

Subjective P-O fit, sometimes referred to as perceived P-O fit, denotes an individuals' direct judgments regarding the extent to which they fit with an organization. In terms of recruitment, subjective P-O fit refers to an overall perception of how well the applicant feels he or she would fit with the organization. In this light, subjective P-O fit assessments can be markedly influenced by the nature of the information provided to applicants while seeking employment. More specifically, research shows that subjective 
P-O fit assessments are greatly affected by both the amount and type of information that organizations are willing to share with applicants (Breaugh \& Starke, 2000; Cable \& Judge, 1996; Judge \& Cable, 1997; Kristof-Brown et al., 2005). Consequently, recruitment material should not only offer job-relevant information, but also information that will help potential applicants assess their relative fit with the organization.

\section{Organizational Attraction}

Since organizational image contributes to an individual's perception of P-O fit, it is understandable that organizational image also impacts job choice attitudes and behaviors which may stem from this initial perception of fit. In other words, organizational image has considerable impact on an applicant's perception of organizational attractiveness and subsequent job choice behavior. Research shows that symbolic attributes, associated with an image, brand, or organizational personality, impact perceptions of attraction (Chapman et. al., 2005; Lievens \& Highhouse, 2003; Slaughter et al., 2004). Similarly, meta-analytic research shows that image is also a strong predictor of job pursuit intentions and job acceptance intentions (Chapman et. al., 2005).

Recent research has even explored the link between image, fit and attraction, showing that organizational personality impacts subjective perceptions of $\mathrm{P}-\mathrm{O}$ fit, which in turn affects organizational attraction (Gregory, 2010). Such findings suggest that the link between organizational personality and attraction is largely driven by an individual's P-O fit perception with an organization (Gregory \& Viswesvaran, 2009). In other words, organizational personality information allows applicants to make inferences about the 
organization, which then allows them to assess the congruence with their own personality, in terms of values, goals, needs, and desires (Highhouse, Thornbury, \& Little, 2007; Kristof, 1996). For example, an individual who is cheerful, friendly, and cooperative will likely search for an organization with similar characteristics, such as organizations high in the Boy Scout dimension. These organizations, such as Disney, place a strong emphasis on being pleasant, attentive to the needs of others, and being family-oriented (Slaughter et al., 2004). On the basis of research, it is likely that this individual would perceive a high congruence with the organization, which would lead them to have a high perception of P-O fit, and ultimately be more attracted to the organization.

Given the clear impact of image on potentially favorable application reactions, practitioners should strive to tailor their recruitment media in a way that clearly projects an accurate and favorable image of the organization. The next section examines the various methods that organizations can choose from when selecting recruitment mediums.

\section{Image and Recruitment Sources}

Given the considerable impact of recruitment throughout the selection process, researchers have devoted considerable attention to the effectiveness of various recruitment methods. These methods, aimed at making individuals aware of job openings, traditionally consisted of newspaper job ads, job fairs, college placement offices, and employee referrals (e.g., Breaugh, Greising, Taggart, and Chen, 2003; Fernandez \& Weinberg, 1997; Rafaeli, Hadomi, \& Simons, 2005). Recently, however, 
there has been a substantial increase in recruitment research (Billsberry, 2007; Breaugh, Macan, \& Grambow, 2008), especially relating to on-line recruitment (e.g. Dineen, Link, Ash, \& DelVecchio, 2007).

Web technology has revolutionized human resource management, particularly in the area of employee recruitment (e.g., Chapman \& Webster, 2003; Lievens, van Dam, \& Anderson, 2002). With over 40 million people turning to the Internet for job searching and over $70 \%$ of organizations practicing Web-based recruitment (Row, 2005), it is clear that Web-based recruitment has become a leading avenue for recruitment efforts (Chapman \& Webster, 2003; Foster, 2003; Hu, Su, Chen, 2006; Zusman \& Landis, 2002). Organizational websites, for example, are becoming a popular recruitment method (Cappelli, 2001) and, therefore, have received a notable amount of attention (e.g., Allen et al., 2007). From the HR practitioner's perspective, websites are viewed as a highly effective recruitment method (e.g., Chapman \& Webster, 2003; Stone, Lukaszewski, \& Isenhour, 2005). Furthermore, organizations are able to easily, and quickly generate a large number of job applicants at a relatively low cost compared to traditional recruitment methods (Rynes \& Cable, 2003).

In addition to organizational websites, companies are now able to communicate with audiences through a variety of other internet-based mediums, specifically Social Networking (SNW) sites. Some of the more commonly used web platforms include Facebook, LinkedIn, Twitter, and interactive blog postings (Jue, Marr, \& Kassotakis, 2010; Shih, 2009). Facebook, a popular SNW, has over 800 million users (facebook.com, 2013) and is expected to increase in popularity in the coming years (Shih, 
2009; Haefner, 2009; Kluemper, Rosen, Mossholder, 2012). Within the Facebook platform, companies are able to create corporate user-profiles to communicate and interact with other users. According to Clara Shih, author of "The Facebook Era", there are over 1.4 million organizations actively using their Facebook page. Similarly, many organizations have openly embraced Twitter, a SNW which allows users to communicate with one another through a series of 140 -character long, real-time 'tweets'. The prevalence of organizations using SNW sites to communicate with external members will only continue to grow as technology develops. It is essential the organizations better understand how they can maximize the effectiveness of these web-based interactions towards employee recruitment.

\section{Image and Corporate Websites}

Given the clear benefits of using internet-based methods for recruitment efforts, research on the role of company websites in applicant attraction has become increasingly popular (Anderson, 2003; Cober, Brown, Levy, Keeping, Cober, 2003; Cober, Brown, Keeping, \& Levy, 2004; Thoms, Chinn, Goodrich, \& Howard, 2004; Williamson, Lepak, \& King, 2003). Just as with any communication medium, web pages offer a variety of ways through which organizations can deliver their desired message. For example, websites can vary not only in terms of the content they provide, but also in the manner through which the webpage is designed (e.g., layout, aesthetics, etc.). Previous research shows that an organization's ability to successfully generate qualified applicants, relies heavily on their ability to effectively communicate employment information (Cappelli, 2001). Therefore, organizations need to be well-advised on how different website 
features may impact applicant choices. Accordingly, there has been a recent increase in research investigating how web pages can be maximized as a recruitment tool (e.g., Braddy, Thompson, Wuensch, \& Grossnickle, 2003; Cober, Brown, Levy, \& Keeping, 2003).

Within this body of literature, researchers have focused on website design features and content-related variables that influence job-seekers' perceptions of an organization's recruitment image, its image as an employer, and job seekers' person-organization (P-O) fit perceptions (e.g., Braddy et al., 2003; Cober et al., 2003). Much of the early research on website design has focused the impact of content, usability, and aesthetics, in terms of predicting applicant attractions (e.g., Cobe et al., 2003; Lyons \& Marler, 2011). Website design features that have been examined include perceptions of attractiveness of recruitment websites in terms of their colors, fonts, pictures, and bulleted versus paragraphs of text (Braddy et al., 2003; Cober et al., 2003; Thoms et al., 2004; Zusman \& Landis, 2002), as well as P-O fit assessment tools (Dineen, Ash, \& Noe, 2002). In terms of website content, previously studied variables include information relating to organizational culture, compensation, and training opportunities (Braddy et al., 2006; Cober et al., 2003).

More recently, researchers have further examined how viewer's impressions of an organization can be changed by viewing an organization's recruitment website (Braddy, Meade, and Kroustalis, 2008). Often, job applicants are limited in their knowledge about the organizations with which they are seeking employment (Rynes \& Miller, 1983). According to signaling theory (Spence, 1973, 1974), when an individual has insufficient 
data, or is undecided in terms of their stance towards a target, he or she will draw inferences on the basis of cues from available information. In the context of recruitment, signaling theory suggests that any information that a job seeker views, may impact his or her impression of the target organization. In this light, Braddy et al. (2008) found that viewing organizational recruitment websites impacted individual's perceptions of organizational favorability, image as an employer, and organizational attractiveness. Furthermore, their results suggest that color, font, and image influence job seekers' perceptions of organizations. However, they acknowledge a need to further investigate these website components in order to be able to provide organizations with clear website design guidelines in order to maximize the Internet as a recruiting tool.

\section{Image and Facebook}

Within the technology revolution, a new form of communication between organizations and individuals is taking place through social media. Jue et al. (2010) define social media as "the many relatively inexpensive and widely accessible electronic tools that evoke anyone to publish and access information, collaborate on a common effort, or build relationships," (p.4). This social media umbrella covers a variety of different SNW mediums such as discussion forums, blogs, wikis, and podcasts. In recent years, SNW use has quickly become the fourth most popular online activity, even surpassing the use of e-mail (Nielsen.com, 2009). Accordingly, organizations have embraced SNW as a means to both communicate with individuals and expand their employee selection efforts. 
Facebook, developed in 2004, recently became the largest SNW site with over 800 million active users across the globe (Treadway \& Smith, 2010). Although the majority of profiles belong to individual users, Facebook also offers the option of creating profile pages for non-personal use such as interests groups, causes, and organizations. As part of this feature, companies are able to create Facebook Pages (distinct from individual profiles) as a way to communicate information about their company with other Facebook users. These Facebook Pages allow companies to extensively-customize their profile with a variety of different components such as interactive dialogue (i.e., 'wall' postings and comments), pictures, videos, business applications, and link to both internal and external sites. Many organizations used Facebook as a recruiting tool as a way to both target potential applicants and to communicate up-to-date information about employment opportunities.

Just as researchers have begun exploring Facebook as a source to assess individuals' personality (e.g., Kluemper et al., 2012; Marcus, Machilek, \& Shultz, 2006), it seems likely that potential applicants could infer an organization's personality through their Facebook presence. According to Funder's (1995) realistic accuracy model (RAM), rating accuracy is enhanced when information is conveyed in a rich, dynamic manner that allows raters to assess behavior over time. In line with the tenets of RAM theory, it is likely that applicants would be able to obtain personality-related information from the SNW profile of an organization in order to form a schema for their corporate personality (Foti \& Lord, 1987). In other words, job seekers could be able to infer the organizational personality of a company by viewing the target organization's Facebook page. 


\section{Differences in Image across Web-based Recruitment Media}

With regards to Web-based recruitment, perceptions of an organization are partly determined by an applicant's ability to infer beliefs about an organization's culture, values, and visions through the company Web site (e.g., Chen, Lin, \& Chen, 2012; Cho \& Lee, 2011). Additionally, research shows that the type and amount of information presented to applicants affects perceptions of P-O fit (Breaugh \& Starke, 2000; Cable \& Judge, 1996; Judge \& Cable, 1997; Kristof-Brown et al., 2005). Accordingly, it likely that variations in the amount and type of information provided to applicants would alter perceptions of organizational image. In other words, applicants could form different perceptions of organizational personality depending on the method of recruitment the individual views (e.g., websites, SNW sites, etc.).

Within this body of literature, researchers have attempted to better understand applicant reactions to organizational websites through content analyses studies. For example, research shows that aesthetics, content, and functionality are all rated as being key components of websites (e.g., Cober, Brown, Keeping, \& Levy, 2004; Allen et al., 2007; Braddy et al., 2006; Williamson, Lepak, \& King, 2003). More specifically, webpages are rated favorably if they include pictures or unique fonts, if they address important job-related attributes, and if they are easy to navigate.

Ultimately, the need to understand differences across communication platforms has become more salient with the increasing popularity of web-based recruiting through both corporate webpages and social media sites. On the basis of this need, scholars should strive to examine how differences in communication media affect pre-hire 
outcomes such as attitudes, intentions and behavior, through differences in organizational personality portrayal. It is clear that job applicants' image perceptions can influence attraction to recruiting organizations, however, little is known about how these perceptions are formed in the web-based context. Given the increasing prevalence of web-based recruitment efforts, it is essential that we further examine the mechanisms through which different internet recruiting methods impact perceptions of organizational personality.

\section{Website Indicators of Organizational Image}

There is a growing body of research examining how aesthetic properties and the content of the information provided in recruitment websites affects applicant reactions (e.g., Dineen, Ash, \& Noe, 2002; Dineen, Ling, Ash, \& Delvechio, 2007; Goldberg \& Allen, 2008; Williamson, King, Lepak, \& Sarma, 2010) and attraction to the organization (e.g., Chen et al., 2012; Cober et al., 2003). However, research in this area is primarily focused on applicant attraction, leaving many unanswered questions about how website components impact applicant perceptions of image.

Recently, a few researchers have shed some light into the new web-based recruitment literature by examining how website features influence applicant perceptions of organizational culture (e.g., Braddy et al., 2006; Braddy et al., 2009). Although distinct from organizational image, research on websites and perceptions of organizational culture has introduced the concept of website components influencing an individual's perception of a company's climate and values. The fundamental idea that applicant perceptions of image could be influenced by website features stems from 
research on signaling theory (Spence, 1974). Rynes (1991) argues that applicants will call on whatever information is available to make inferences about unknown organizational attributes when faced with limited information. For example, characteristics pertaining to website content, layout and pictures could be used as indicators of the organization's image as a whole. Furthermore, researchers have found that both website features and content pertaining to organizational values, policies, awards, and goals affected viewers' perceptions of organizational culture (Braddy et al., 2006; Braddy et al., 2009).

\section{Current Study}

Given the surge of technological advancements, there is a need for research exploring how potential applicants form perceptions of image from web-based recruitment media. Early research in this area has focused on the impact of website features on attraction (e.g., Braddy et al., 2006; Braddy et al., 2009) with little attention given to how perceptions of image are affected. A goal of the present dissertation is to identify aspects of web-based recruitment media that influence viewer perceptions of image, specifically operationalized as organizational personality. The present research examines objective components of corporate websites and organizational Facebook profiles that shape perceptions of organizational personality dimensions. Specifically, we examine how objective indicators of image impact perceptions of image in web-based recruitment materials. These objective indicators will be key to having a better understanding of how image perceptions are influenced by web-based recruitment media. 
Given the tremendous impact of image in the recruitment process, in terms of perceived fit and attraction to an organization, it is essential that organizations project accurate and consistent depictions of their organizational image across all of their recruitment methods. Consequently, the current research also examines whether image assessments vary by different web-based recruitment methods, both through subjective and objective assessments.

Lastly, it is beneficial for organizations to be aware of, not only what image they are projecting, but also how they can best manage and modify these image projections. Therefore, a final goal of this dissertation will be to identifying which objective indicators are most influential for projecting specific image dimensions.

\section{Summary}

In sum, given the lasting impact of image on potential applicants and the rising popularity and use of web-based recruitment media, it is necessary for scholars to examine how these new recruitment methods impact perceptions of organizational image. The present research seeks to extend the literature by identifying objective components in recruitment media, specifically corporate websites and company Facebook profiles that indicate a particular organizational personality dimension. Furthermore, the subjective perceptions and objective assessments of image are examined across different types of recruitment media. Lastly, website characteristics are assessed in terms of relative impact on image perceptions in an effort to inform organizations on how to best manage image projections in the recruitment context. The following section will provide a 
comprehensive review of organizational image, trends in web-based recruitment, and viewer perceptions of and reactions to web-based recruitment media. 


\section{Chapter II: Literature Review}

The second chapter of this dissertation will provide a comprehensive review of the organizational image literature. First, a review of the many, and often overlapping, conceptualizations of image are presented. Second, the mechanisms through which image affects business outcomes are discussed, particularly in the realm of employee recruitment. Third, recruitment is examined in further detail, including a general overview, current trends in web-based recruitment methods, and how these methods impact perceptions of organizational image. Finally, details pertaining to the present study taken from relevant empirical and theoretical findings are provided, culminating in the research questions and hypotheses under study.

\section{Image}

At its core, organizational image refers to people's overall impressions of an organization. Lievens (2006) describes it as the "net cognitive reactions and associations (p. 569)" of individuals which together form bodies of knowledge and opinions about an organization. Many researchers agree that an organization is not associated with a single image, but rather multiple images from different stakeholders that may not always coincide. Employees, investors, customers and potential applicants will each form their perceptions of an organization's image on the basis of their unique experience with and exposure to the organization. For example, a customer's perception of a company's image as a provider of goods and services will likely differ from the image perceptions of a current employee at the same company. 
Dutton et al. (1994) provide a framework of image as two complementary, but distinct components. The framework is built on the underlying assumption that insiders and outsiders of an organization have access to different information about the organization. Additionally, because of the inherent differences in the nature of the relationship with the organization, each group evaluates the information on the basis of their own goals and values. The first component operationalizes image as what the organizational member believes is "distinctive, central, and enduring" (p. 239) characteristics of the organization (Dutton et al., 1994). This conceptualization is frequently associated with terms such as perceived organizational identity (Albert \& Whetten, 1985; Dutton et al., 1994) and organizational culture (O’Reilly, Chatman, \& Caldwell, 1991; Schein, 1990). In the second component, image represents the way members of an organization believe outsiders view the organization (Dutton \& Dukerich, 1991). Recently, the operationalization of organizational image has shifted from members' opinions of outsiders' image perceptions, to simply outsiders' image perceptions of the organization (e.g., Allen et al., 2007; Lievens et al., 2007). The present study will focus exclusively on the second component of image, which views image as a function of the external members' perceptions of image.

Over the years, researchers have offered numerous variations on the specific scope and definition of outsider-focused image, such as construed external image (Dutton et al., 1994), projected image (Bernstein, 1984, Whetten, Lewis, \& Mischel, 1992), desired future image (Goia \& Thomas, 1996), transient impression (Berg, 1985; Grunig, 1993), corporate reputation (Fombrun, 1996), corporate identity (Olins, 1989; van Riel \& 
Balmer, 1997), employer branding (Schneider, 2003), organizational image (Lievens \& Highhouse, 2003; Lievens, 2006), and organizational personality (Slaughter et al., 2004).

The following section covers specific details, similarities, and discrepancies across each of the image conceptualizations listed above. First, conceptualizations formed primarily on internal perceptions are presented, followed by definitions emphasizing external perceptions of image, then a brief discussion of conceptualizations stemming from the business and marking literature, lastly unifying conceptualizations providing frameworks for assessing image components are introduced.

\section{Internally-Focused Conceptualizations of Image}

Construed External Image. As mentioned above, construed external image refers to an internal members' own assessment and beliefs of how external members view an organization (Dutton et. al., 1994). An example of this concept is how an employee thinks an external client perceives the organization. This type of image evaluation not only provides information about the social evaluation of the organization as a whole, but also sheds light on how external members perceive internal members who are affiliated with the organization. A key element of this conceptualization is the notion that internal members may possess distorted perceptions of how external members view the organization. Inaccurate, or distorted, perceptions may be particularly prevalent within upper-management when dealing with unforeseen changes in the market, ethical dilemmas, and issues relating to organizational integrity (e.g., Ginzel, Kramer, and Sutton, 1993). 
Projected Image. Contrary to the notion that upper-management is susceptible to distorted image perceptions, Whetten et. al. (1992) argue that projected image is the way "organizational elites" would like external members to view the organization. Whetten bet al.'s viewpoint highlights upper-management's desire to project an image that stems from the organizations' identity. More specifically, an organization's identity refers to core and enduring organizational characteristics that distinguish it from other organizations (Albert \& Whetten, 1985). By aligning the projected organizational image with the internal identity, the projected image serves as a way to relay vital features of an organization to outside members. At the same time, it is possible that uppermanagement's projected image may be influenced by social desirable-driven impression management techniques, over-emphasizing the positive features and even intentionally disguising an organizations' true identity (Gioia et al., 2000). This idea is consistent with Bernstein's (1984) idea that image should be defined as a product of public impressions generated to appeal to outsiders. According to this, in fact, the projected image could not only be a slightly tailored projection of reality, but an entirely fabricated entity.

Desired Future Image. As with projected image, desired future image is also characterized as being driven by top management. A key distinction is that this image is developed on a genuine future vision of the organization (Goia \& Thomas, 1996) as opposed to methodically, and possibly deceptively, selected features of the current organization's identity. This conceptualized image also serves as a way to communicate the desired future vision to internal members of the organization. 


\section{Externally-Focused Conceptualizations of Image}

Transient Impression. Berg (1985) was the forerunner of exploring image as a product of how outsiders perceive the image of organization-omitting the component of how internal members think the external members to perceive it to be. More specifically, Berg believed external members shape their image impressions mainly in response to a particular action or event associated with the organization. As implied by the name, transient impression is characterized as a more temporary, event-focused conceptualization of image compared to other definitions. Closely related to this conceptualization, Grunig (1993) explains image as the impression constructed by external members through direct observation or interpretation of a message, often including symbols, provided by the organization.

Corporate Reputation. In line with the conceptualization of image as purely focused on outsiders' perceptions, Fombrun (1996) defines corporate reputation as the collective impressions of an organization's actions and accomplishments, as perceived by members external to the organization. Corporate reputation is in sharp contrast to transient impression, in the sense that reputation is characterized by enduring, comprehensive judgments over an extended period of time (Gioia, et. al., 2000). It is necessary to note, however, that the term 'corporate reputation' spans numerous disciplines and academic subject areas (e.g., economics, marketing, organizational behavior, etc.), each with their slight nuances in terms of definition (Fombrun \& van Riel, 1997). In recent years, researchers have tried to integrate these different areas of research to create a more unified understanding of corporate reputation. One of these more 
contemporary definitions, for example, defines it as “observers' collective judgments of a corporation based on assessments of the financial, social, and environmental impacts attributed to the corporation over time" (Barnett, Jermier, \& Lafferty, 2006, p. 34). Ultimately, despite slight definitional inconsistencies across disciplines, the elements of externally-formed perceptions and global assessments remain core to the definition.

\section{Image Conceptualizations in the Business Literature}

Corporate Identity. Outside of the realm of organizational behavior research, the field of public relations and marketing often refer to image in terms of corporate identity. In this business-focused body of literature, researchers concentrate on the idea of companies projecting information to stakeholders as a way to achieve strategic goals (Olins, 1995). As such, the term corporate identity is closely tied to visual representations of the organization, specifically through the design and use of corporate symbols and logos (Hatch \& Schultz, 1997; Olins, 1989). Unlike projected image, van Riel and Balmer (1997) and Hatch and Schultz (1997) maintain that this projection is important for internal and external constituents, thus spanning a broader audience.

Employer Branding. Based on marketing research, a brand refers to "a name, term, sign, symbol, or design, or combination of them which is intended to identify the goods and services of one seller or group of sellers and to differentiate them from those of competitors" (Schneider, 2003). In traditional brand management research, Park, Jaworski, and MacInnis (1986) conceptualize brands as mapping onto three categories of the consumer needs they fulfill: (a) functional needs, (b) symbolic needs, and (c) experimental needs. Functional brand refers to objective, physical, and tangible 
characteristics of a product. Symbolic aspects describe the product in terms of subjective, abstract, and intangible features. Lastly, experiential brand concepts refer to the product's effect on sensory satisfaction or cognitive stimulation. This marketing framework, with a primary focus on functional and symbolic needs, has been applied to an employee selection context.

Recently, employer branding has emerged from applying traditional marketing brand principles to the field of employee recruitment (Cable \& Turben, 2001; Capowski, 1997; Maurer, Howe, and Lee, 1992). Early adopters of employer branding, Ambler and Barrow (1996), suggest that employer brand relays critical information about the organization such as personality and differentiation for potential applicants. Backhous and Tikoo (2004) define employer branding as the process of building an identifiable and unique employer identity in an effort to highlight the organization's unique employment offerings and environment. In other words, it can be described as an organization's efforts to project the organization as a desirable place to work for both existing and prospective employees (Lloyd, 2002). More recently, researchers have begun forming multidisciplinary frameworks to achieve a better understanding of image and its components.

\section{Unifying Conceptualizations of Image}

Organizational Image. Embracing the idea of image as a long-lasting, perception of an organization, and influenced by the fundamentals of brand management, Lievens (2006), defines organizational image as people's global impressions of an organization, comprised of "loose structures of knowledge and beliefs about an 
organization (p. 569)." In addition to representing overall perceptions, it also serves as a way for external members (e.g., customers, investors, employees, applicants, etc.) to categorize, store, and recall relevant information about an organization. These impressions are not only shaped by communication from the organization itself (e.g., advertising, sponsorships, etc.) but also by other sources such as media coverage and general societal perceptions. Although it changes slowly over long periods of time, an organization's image is dynamic and capable of evolving, generally at the discretion of the internal members. For instance, if an organizations wishes to modify their image, it would be necessary to first identify which factors are contributing to the current image perceptions across all of the stakeholders. Once the organization is able to identify how the image is being shaped, internal members can work to either highlight or tailor these factors in order to project the desired image.

Stemming from brand equity theory (Aaker, 1997), Lievens and Highhouse (2003) introduced the Instrumental and Symbolic Framework for describing image, which appropriately states that an organization's image can be organized into two types of attributes, instrumental and symbolic. Instrumental attributes refers to objective features, or characteristics, individuals associate with an organization. These attributes may range from factual or historical aspects of an organization, to more specific organizational practices or guidelines. Research shows that applicants, for example, may know some instrumental attributes, such as size of the organization, benefits offered, and career development options, prior to applying to a specific job (Lievens \& Highhouse, 2003; Lievens, 2006). Symbolic attributes, on the other hand, refer to trait-related inferences about the organization. There are two key distinctions between these two types 
of attributes. First, in sharp contrast to objective instrumental attributes, symbolic attributes are subjective, abstract, and intangible attributes associated with an organization. Second, they express symbolic information through imagery that can be associated with the organization. Symbolic attributes, for example, refers to using words such as trendy and honest to convey human-like personality characteristics on the organization. Research has shown that applicants are able to meaningfully and reliably assign symbolic attributes to organizations (Lievens \& Highhouse, 2003; Lievens et al., 2005).

Organization Personality. In line with the instrumental-symbolic framework for organizational image, scholars further developed the idea of organizations being associated with trait-like personality characteristics. Slaughter et al. (2004) define organization personality (OP) as "the set of human personality characteristics perceived to be associated with an organization (p. 86)." In previous personality research, the term personality has often referred to two distinct conceptualizations: one referring to a person's internal processes, usually to explain why he or she acts a certain way; the second concerned with how one is perceived by family, friends, and coworkers, or social reputation (Hogan, 1991). Slaughter and colleagues specifically operationalize personality as the manner in which the organization is perceived by outsiders (i.e., 'social reputation') as opposed to referring to its internal processes. More so, organization personality is shaped by the different ways the organization presents itself as well as how other entities present the organization to the public. Examples of these possible channels for organization personality projections include television and radio advertisements, media coverage and press releases, the Internet, personal familiarity with the 
organization's place of business or clientele, and company-related information gathered from ones' friends and family (Slaughter et al., 2004). A key feature of organization personality is the notion that outsiders are able to make an assessment of personality, regardless of the amount of exposure they have had to the organization-assuming there is at least some, even if it is very limited (Lievens \& Highhouse, 2003; Slaughter et al., 2004).

Lieven et al.'s (2006) study sought to not only capture the structure of organization personality, but also to develop and validate a self-report measure of organization personality perceptions. The research was conducted under the assumption that, just as individuals ascribe personality traits to themselves (Ashforth \& Mael, 1989), to other people (Hogan, 1991; Norman \& Goldberg, 1966; Watson, 1989), and product brands (Aaker, 1997; Siguaw, Mattile, \& Austin, 1999), organizations could also be described in trait-terms. Brand personality, defined as "the set of human characteristics associated with a brand" (Aaker, 1997, p. 347), is accepted by marketing researchers and consumers as an effective way for describing brands (Siguaw et al., 1999). Aaker's research suggests that brand personality encompasses five broad dimensions of personality: Sincerity, Excitement, Competence, Sophistication, and Ruggedness. Research shows, for example, that people generally view Coca-Cola as cool and allAmerican (Pendergrast, 1993), while Pepsi is perceived as being young and exciting (Plummer, 1985). Lievens and Highhouse (2003) also organized symbolic attributes (see above) into similar dimensions. Specifically, they expanded on Aaker's (1997) brand framework for a total of five dimensions: Sincerity, Innovativeness, Competence, Prestige, and Robustness. 
Together, these two streams of research shaped the foundation for the five dimensions of perceived organization personality (Slaughter et al., 2004), which consists of Boy Scout, Innovation, Dominance, Thrift, and Style. The Boy Scout dimension refers to perceptions of an organization's honesty, helpfulness, attentiveness, friendliness and family-orientation. Organizations perceived to be strong on the Boy Scout dimension are Target, Disney, and Johnson \& Johnson. The second dimension, Innovation relates to how unique, interesting, or creative an organization is viewed. Organizations found to be perceived as highly innovative include IBM, PepsiCo, and Microsoft. The Dominance dimension corresponds to an organization being associated with success, popularity, or high-activity levels. Organizations perceived to be strong on the Dominance dimension include Coca-Cola, general Motors, Disney, and AT\&T. Thrift refers to organizations that are seen as low budget, small, or sloppy. Organizations perceived as being strong in this dimension include K-Mart, Kroger, Wal-Mart, Subway, and J. C. Penney. Lastly, perceptions of "hipness," contemporary, or trendy are indicative of the Style dimension. Past research indicates that Nike, Reebok, Pepsi, and Motorola are rated highly on this dimension (Slaughter et al., 2004). It is important to note that organizations are not perceived as depicting only a single OP dimension, but rather varying levels of each OP dimension.

Scholars generally agree that image is an impression that develops from a loose combination of facets and feelings (e.g., Belt \& Paolilo, 1982; Gatewood, Gowan, \& Lautenschlager, 1993). Accordingly, organizational personality perceptions are one source of these feelings about an organization and can be viewed as one component of an organization's image (Slaughter \& Greguras, 2009). In line with recent research and 
because of the comprehensiveness of the conceptualization, the present research operationalizes organizational image using Slaughter et al.'s (2004) definition of organizational personality. The next section of this dissertation will discuss the importance of organizational image, particularly in terms of how it impacts business processes.

\section{Consequences of Image}

Given the extensive amount of research on defining and conceptualizing organizational image, it is evident that an organization's image has the potential to influence a number of organizational outcomes. The nature of these outcomes is largely determined by who is doing the perceiving. In other words, the business processes that are impacted depend on the role of the image-receiving stakeholder. For example, image perceptions may invoke different responses depending on whether the external member is an investor, customer, or potential applicant (Lievens, 2006). Some of the various, potentially favorable, consequences for organizations are discussed below.

One of the ways image can impact business processes is through image perceptions by current and potential investors. If investors perceive an organization's image to be desirable, the organization will likely see positive outcomes such as continued or increased investments from existing investors, and an increase in interested

new investors. As such, organizations may gain competitive leverage, which contributes to the competitive ability of the firm in the general market (Lievens, 2006; Lyons \& Marler, 20111). 
Customer image perceptions are another way that organizations can see positive results. More specifically, an abundance of marketing research shows that an organization's image affects consumers' product choices (Deephouse, 2000; Howard \& Sheth, 1969). Such findings suggest that perceptions of image for the overall organization may signal to customers, information relating to product quality, cost, or customer-services skills (Deephouse, 2000; Lievens, 2006).

Lastly, image perceptions can have monumental implications for potential applicants, specifically relating to how attracted applicants feel towards an organization as a desirable place to work (Lievens \& Highhouse, 2003; Turban \& Cable, 2003). The impact on perceptions of attraction is particularly true for the early stages of recruitment when applicants have the least amount of knowledge about the job, or the organization itself. At this point, potential applicants are forced to rely heavily on their limited knowledge in order to shape their overall impressions of the organization. These initial impressions, or image, have a large impact on whether or not the potential applicant will actually pursue employment with the organization. At its core, applicant perceptions of organizational image impact levels of attraction to the organization, which in turn impact employment decisions during the recruitment process. As such, organizations with more favorable perceptions are able to attract more, and potentially better qualified applicants, than their less favorable counterparts (Lievens, 2006). It is important to highlight that image perceptions impact applicants during the duration of the recruitment process, not simply in the early stages (Uggurslev et al., 2012). Research shows that impressions of organizational image at the early stages of recruitment were strong predictors of 
applicants' attraction to the organization at later phases, such as on-site interview and final job acceptance decisions (Lievens, 2006).

The present research is primarily focused on how image perceptions are formed from the perspective of potential applicants during the early phases of the recruitment process. The following sections cover relevant theories that help explain how image perceptions influence potential job applicants, specifically relating to organizational attraction.

\section{Image and Applicant Attraction}

By applying the instrumental-symbolic framework to organizational image, research has shown that symbolic attributes (i.e., trait-related inferences about an organization) impact feelings of attraction towards an organization above and beyond instrumental attributes (Lievens, 2007; Lievens \& Highhouse, 2003). Furthermore, applicants are better able to differentiate among employment opportunities when symbolic images are considered than if symbolic images are not present (Lievens \& Highhouse, 2003; Slaughter et al., 2004).

In a 2004 study, Slaughter et al. found that organizational personality was significantly related to perceived organizational attractiveness, job pursuit intentions, and the likelihood that applicants would accept job offers. When examining differences among the personality dimensions, they found that Dominance and Thrift (negative relationship) were the factors most strongly, and consistently, related to organizational attraction. 
Recently, Uggurslev et al. (2012) conducted a meta-analysis to examine the relative strength and incremental variance accounted for by seven different recruiting predictors (e.g., job characteristics, organizational characteristics, recruiter behaviors, recruitment process characteristics, fit perceptions, hiring expectancies, and perceived alternatives) on applicant attraction. On the basis of 232 studies, results showed that characteristics of the organization, such as organizational image, accounted for unique variance in applicant attraction at multiple stages of the employee selection process. More importantly, applicant perceived fit with the organization was the strongest predictor of applicant attraction.

Research on organizational image suggests that job seekers prefer companies with favorable images, even over a company who may be offering a similar job but yields a less favorable image perception (Lievens \& Highhouse, 2003; Turban \& Cable, 2003). Yet, how exactly does an individual determine whether the perceived image of an organization is favorable or unfavorable? The next section covers theories and frameworks to help clarify how individuals determine whether or not an organization's image is 'favorable' for them.

\section{Person-Organization Fit and Attraction}

Potential applicants consider features beyond just salary and benefits when determining whether or not an organization would be a desirable place to work. Beyond tangible features of the job, it is crucial that individuals feel a level of congruence between their values and beliefs, and those of the organization. Research has consistently shown the impact of applicant personality and organization image similarity on 
organizational attraction (Devendorf \& Highhouse, 2008; Schneider, 1987; Tom, 1971; Turban \& Cable, 2003; Turban \& Keon, 1993).

At the global level, person-environment is defined as the compatibility between individual and work environment characteristics (Kristof-Brown et al., 2005). Underneath the person-environment umbrella, researchers have studied a variety of different manifestations, such as person-organization fit and person-job fit. One of the most commonly examined aspects of person-environment fit is subjective personorganization (P-O) fit. Subjective P-O fit, or perceived P-O fit, refers to an individuals' direct judgments concerning the extent to which they are compatible with an organization (Judge \& Cable, 1997; Kristof, 1996; Kristof-Brown et al., 2005). In the context of personnel recruitment, Kristof (1996) describes subjective P-O fit as the overall evaluation of how well the job seekers' values fit with the attributes of the organization. Research on this topic generally suggests that individuals prefer to work for an organization whose qualities align with their own values (Cable \& Judge, 1996; Kristof, 1996; Schneider, 1987), goals (Vancouver \& Schmitt, 1991; Witt \& Nye, 1992), and personality (Christiansen, Willanova, \& Mikulay, 1997; Ryan \& Schmidt, 1996). Although most research examines this phenomenon after organizational entry (Kristof, 1996; Kristof-Brown et al., 2005), or in an experimental setting (e.g., Lievens, Decaesteker, Coetsier, \& Geirnaert, 2001; Turban \& Keon, 1993), the few studies examining P-O fit at the recruitment level show similar results (Dineen et al., 2002; Hu et al., 2007; Judge \& Cable, 1997). More specifically, P-O fit for employees has been found to strongly influence organizational commitment, intentions to quit, and organizational satisfaction on the job (Kristof-Brown et al., 2005) whereas P-O fit for job 
seekers has a strong influence on organizational attraction, job acceptance rates, and job acceptance intentions (Chapman et al., 2005; Kristof-Brown et al., 2005).

In one of the studies using job seekers and actual recruiting organizations, Judge and Cable (1997) examined the link between Big Five personality dimensions and preferences for organizations with varying organizational cultures. In addition, they examined subjective impressions of P-O fit in relation to applicant attraction. Results suggest that the Big Five personality traits (i.e., Neuroticism, Extraversion, Openness to Experience, Agreeableness, and Conscientiousness) were related to their similar counterparts in the organizational culture dimensions. Additionally, they found that both objective fit (i.e., congruence between culture preference and organizational culture) and subjective fit (applicant's direct perception of fit) were related to increased attraction to organizations.

More recently, the relationship between perceived fit and applicant attraction has been examined using symbolic, or personality-trait based, attributes for organizations. Schreurs, Druart, and Proost (2009) examined the moderating role of the Big Five personality factors in the relationship between five trait-based inferences about organizations (Sincerity, Excitement, Competence, Prestige, and Ruggedness) and organizational attractiveness. Results showed that Sincerity was positively related to attraction only for those individuals high on Conscientiousness, and a similar relationship for Excitement and attraction for individuals high on Openness to Experience. Similarly, Slaughter and Greguras (2009) measured perceived fit as congruence between and individual's personality, and their perceptions of an organization's image using their 
validated organizational personality dimensions. Results were consistent with previous findings, showing that congruence between Conscientiousness and Boy Scout, as well as Openness to Experience and Innovation, were found to be significant predictors of organizational attraction. Additionally, results consistently showed that poor fit was related to low attraction levels, suggesting that perhaps lack of fit may be more damaging than strong fit is helpful (Slaughter et al., 2009). Although continued research on applicant fit perceptions and attraction are warranted, results overwhelmingly suggest that good fit, or lack of poor fit, is related to increased organizational attraction.

\section{Image Congruity Theory and Attraction}

According to Markus and Nurius (1986), individuals conjure thoughts of their ideal selves which are rarely similar to descriptions of their actual selves. Image congruity theory uses the concept of multiple self-images (i.e., actual self, ideal self) to explain consumer decision making. Image congruity theory, in essence, posits that consumers buy products to portray images of how they would like to appear (Rogers, 1951; Sirgy, 1985). As such, consumers are motivated to choose options that are consistent with their actual self-image as well as their ideal self-image. On the basis of which ideal most closely matches their choice, individuals will either experience self congruity (i.e., congruence with actual self) or ideal congruity (i.e., congruence with ideal self). Research shows that self congruity and ideal congruity have been used to predict consumer purchase motivation, intentions, and preference (Ericksen, 1996; Sirgy, 1985; Sirgy et al., 1997). 
More recently, Nolan and Harold (2010) have applied the congruity theory in the context of applicant decision making. Specifically, they examined the role of image congruity in the recruitment process by providing applicants with a series of different job advertisements, each tailored to project a different organizational image. In accordance with the tenets of image congruency theory, they found that prospective job seekers were attracted to organizations with personalities perceived to be similar to either own actual and ideal-self concepts.

\section{Self-Continuity and Attraction}

Comparable to image congruity theory, Steele (1988) contends that people generally want to maintain the continuity of their self-concepts over time and across situations. As such, a job seekers perception of an organization will either add to or subtract from this continuity, depending on whether or not the individual believes it to be relevant to his or her self-concept over time. In other words, similarity between the selfconcept and perceived organizational entity (i.e., image) enhances continuity. Enhanced continuity of self will then strengthen a member's identification by making the perceived organization more attractive. Dutton et al. (1994) argue that this can be explained in two ways. First, people perceive image congruence as more attractive simply because similar, or familiar, information is easy to process and understand. According to social psychologists, individuals attend to and process information that is "self-relevant" more easily than "self-irrelevant" information (Markus \& Wurf, 1987). Second, individuals are drawn to organizations that they perceive to be similar to themselves because it provides easy opportunities for self-expression (Shamir, 1991). Dutton et al. (2004) elaborate that 
job seekers are drawn to organizations that will allow them to enact a fuller range of characteristics and values in their self-concept. For instance, an applicant who is environmentally conscious and values sustainability, will be more drawn to an organization that endorses green-initiatives in the workplace than an applicant who does not share those values.

Having covered the many conceptualizations of image, how image affects business outcomes, and how image perceptions specifically impact applicant attraction towards the organization, the next section will turn to a more focused look at recruitment and image projections.

\section{Recruitment and Image}

In the early stages of recruitment, potential applicants have very limited knowledge of the large number of jobs and organizations that they generate for future consideration (Barber, 1998; Turban, 2001). Early impressions of an organization's image have been shown to be a strong predictor of continued applicant attraction in the later phases of the recruitment process (Turban, Forret, \& Hendrickson, 1998; Powell \& Goulet, 1996). Consequently, companies should be particularly aware of the sources of information applicants are using for shaping their initial image perceptions. The following section will cover the basic tenets of the recruitment process, current trends in web-based recruitment, and detailed looks at different internet methods commonly used for recruiting applicants. 


\section{Recruitment Overview}

Given the high amount of resources being spent on recruitment and the impact it has on perceptions of image and overall business success, it is imperative that practitioners understand how to best leverage their assets when designing and implementing their recruitment efforts. Most can agree that the primary purpose of recruitment is to identify and attract potential employees. However, researchers often vary in terms of the specific tasks and responsibilities that fall within the recruitment domain. Rynes (1991), for example, defines recruitment as "encompassing all organizational practices and decisions that affect either the number, or types, of individuals who are willing to apply for, or to accept, a given vacancy" (p.429). Similarly, Breaugh (1992) states that recruitment consists of "those activities that (1) influence the number and/or types of applicants who apply for a position and/or (2) affect whether a job offer is accepted" (p.4). Still, some argue that such definitions are too broad and advocate a more structured definition that provides more practical guidelines for organizations. More specifically, these broad definitions are criticized for combining the recruitment process with recruitment outcomes, which can lead to confusion and misinterpretation when evaluating their effectiveness. For example, recruitment efforts would only be recognized as recruitment if they lead to increased applicant attraction, whereas those interventions that were unsuccessful in attracting applicants would not fall under the recruitment umbrella (Barber, 1998).

Accordingly, in an effort to disentangle the process from the outcome, Barber (1998) defines recruitment as "those practices and activities carried out by the 
organization with the primary purpose of identifying and attracting potential employees (p.5).” Barber organizes recruiting into three phases: (1) generating applicants, (2) maintaining applicant status, (3) and job choice. The first phase, or early recruitment stage, involves reaching out to the population, or part of the population, to apply for the position. In the second phase, organizations persuade applicants to remain interested in the organization as the selection process unfolds. Lastly, the job choice stage involves the organization attempting to persuade desirable applicants to accept job offers. Although each phase is integral for a successful recruitment process, the early recruitment stage is critical for selection effectiveness by generating the initial set of applicants to go through the subsequent stages (Carlson, Connerley, \& Meacham, 2002). This research is particularly interested in how perceptions of organizational image are formed in the early phases of recruitment.

\section{Web-Based Recruitment of Job Applicants}

A central activity of recruitment, especially in the early stages of the process, is communicating information about jobs, working conditions, expectations, values, and climate in an effort to encourage job seekers to pursue employment with the organization (Popovich \& Wanous, 1982). Communication processes, by definition, require a sender (e.g., recruiting organization), a receiver (e.g., potential applicants), message content (e.g., recruitment information), and a communication medium (Jackson, 1992). As previously mentioned, traditional mediums of communication include newspaper job ads, career fairs, head hunters, and employee referrals. Over the past decade, however, organizations have shifted their focus from traditional methods to web-based recruitment 
as a major medium for recruitment (Berry, 2005; Chapmen \& Webster, 2003; Foster, 2003; Zusman \& Landis, 2002).

Organizations are increasingly turning to the Internet as a primary choice for recruitment communication (Cappelli, 2001; Lievens \& Harris, 2003). For example, it is estimated that over $80 \%$ of large organizations have official recruitment web pages (Capelli, 2001; Kaminski, 2010). These web-based tools provide numerous advantageous over traditional recruitment mediums, most markedly perhaps, is their significantly reduced cost while reaching a wider applicant pool (Cober, Browm, Blumental, Doverspike, \& Levy, 2000). Equally as powerful, these web-based mediums have the potential for more immediate and dynamic communication styles with job seekers than traditional print media (Leong, Stanners, \& Huang, 1998; Pavlou \& Stewart, 2000). Enhanced communication could be a reason why individuals are also showing a preference for the Internet over traditional methods when it comes to applying for jobs (Zusman \& Landis, 2002). Finally, there is a growing body of research showing that recruitment websites play a pivotal role in attracting not just more applicants, but more qualified applicants for organizations (Allen et al., 2007; Cober et al., 2004; Dineen et al., 2007).

\section{Web-Based Recruitment Methods}

There are three primary recruitment methods that organizations can choose from to relay information and communicate with job seekers. One method is the use of job boards, or job listing websites (e.g., Monster.com, HotJobs.com), where organizations can post information about job openings through external third parties. These function 
similarly to a newspaper advertisement, except that they are in a web-based format. Job boards provide applicants with added conveniences, such as a centralized repository for job postings across different organizations and recommended job posting based on their user profile preferences. While these third-party websites can substantially increase the number of viewers reading the job advertisement, potentially reaching a more geographically diverse group of applicants (Crispin \& Mehler, 1997), they provide limited information about organizations beyond traditional recruitment media (Kroustalis, 2009). The limited information for prospective applicants is likely due to the fact that most third-party sites change incremental fees based on the amount of content listed in the advertisement. Additionally, interested applicants generally have to apply to the hiring organization indirectly through the third-party website, thus completely bypassing the hiring organization's website altogether (Zusman \& Landis, 2002).

Research shows that at least $93 \%$ of the firms in North America actively use their own website to recruit applicants (Cober et al., 2000; Lievens \& Harris, 2003; Zusman \& Landis, 2002). Corporate websites, a primary tool used by organization, generally provide much more information about the organization compared to traditional recruitment media and Internet job boards (Cappelli, 2001; Lievens \& Harris, 2003). Embedded within the organization's main website, most companies provide a 'careers' section dedicated to providing job seekers with information about career opportunities and job openings. These websites have several advantages, for both organizations and job seekers. Compared to job boards, content can be posted at a much lower cost, and with less space restrictions (Braddy et al., 2003). Although specific content varies, research shows that companies usually provide information regarding the culture of the 
organization, organizational policies, mission and value statements, employee testimonials, and information regarding benefits, rewards, and organizational programs and initiatives (Cober et al., 2000). Ultimately, the goal of this information is to provide an accurate and positive impression of the organization for the viewer (Gatewood et al., 1993). In terms of the prospective applicant, the increase in content allows for more information about the organizational image, which may facilitate better employmentrelated decisions (Braddy et al., 2006). Ultimately, the use of corporate websites for recruitment activities will likely only continue to increase in the coming years.

Even more recent, organizations have started exploring social networking (SNW) sites as a potential medium for recruitment activities. This is not surprising given that SNW has quickly become the fourth most popular online activity, even surpassing email (Nielsen.com, 2009). Although using SNW sites as a source for applicant information is strongly cautioned due to a lack of systematic evaluation (Davison, Maraist, \& Bing, 2009; Schings, 2009), organizations are increasingly marking their territory in the SNW realm (Barnes, Lescault, \& Andonian, 2012). Technically speaking, SNW sites (or social media) refers to "a group of internet based applications that builds on the ideological and technological foundations of Web 2.0 and it allows the creation and exchange of usergenerated content (Kaplan \& Haenlin, 2010, p. 61).” At their essence, SNW sites are web-based communities that facilitate the posting and exchange of information such as pictures, music, videos, blogs, and sharing links. Although the purpose and user demographic varies across sites, the most wide-reaching and popular is Facebook. Initially developed in 2004 solely for Harvard students, Facebook evolved and opened its eligibility to anyone with a valid email address. Today, Facebook alone has over 955 
million active users who log on at least once every 30 days - half of which log on every day. Additionally, Facebook now offers the option of creating a profile page for businesses, local company or even a brand (www.facebook.com, 2013). As of 2012, it was estimated that $68 \%$ of Fortune 500 companies actively used their Facebook page, an $8 \%$ increase from the previous year. This includes eight of the top ten companies, such as Wal-Mart, Chevron, ConocoPhillips, General Motors, General Electric, Fannie Mae, Ford Motors and Hewlett-Packard (Barnes et al., 2012). These business profiles allow firms to easily build an audience, market products and services, share information about employment opportunities and events, interact with other users, and connect to other social media outlets.

A few empirical studies (e.g., Hsu \& Tsou, 2011; Laroche, Reza, Habibi, \& Richard, 2012) have employed marketing and branding principles to assess how organizations use this new media to communicate with external members. Within the research, brand communities have been identified as a "non-geographically bound community based on a structured set of relations among admirers of a brand (Muniz \& O’Guinn, 2001, p.412)." Scholars argue that firms, such as Jeep and Harley Davidson, have formed strong social communities online where they can communicate and interact with members in order to solidify their brand (Anderson, 2005; Kaplan \& Haenlein, 2010). Although research is still sparse, an environment such as Facebook, provides an ideal opportunity for organizations to project their image to potential applicants. 


\section{Webpage Design and Perceptions of Attraction}

Even with the increasing popularity and increased benefits of web-based recruitment for attracting applicants, organizations are still unclear as to how they can maximize the effectiveness of these new tools. There is a growing body of research examining how aesthetic properties and the amount and type of information provided affects job seekers' reactions to the websites (e.g., Dineen et al., 2002; Dineen et al., 2007; Goldberg \& Allen, 2008; Williamson et al., 2010). Unfortunately, a majority of this research has been conducted using fictitious company websites, which has limited the ability to fully examine all the factors that play into web-based recruitment (Allen et al, 2007; Cable \& Turban, 2001; Rynes, 1991).

Since a major goal of applicant recruitment at the early stages is to enlarge the potential qualified candidate pool, a significant amount of research has focused on how the design elements of recruitment websites influence applicant attraction (Anderson, 2003; Hu et al., 2006). Within this body of research, scholars have examined the influence of content, usability, aesthetics, speed, and attractiveness of the material. The consensus from this research is that both content and style of an organization's web page can influence the company's attractiveness to applicants (Cober et al., 2003; Williamson et al., 2003; Chen et al., 2012).

For example, Cober et al. (2003) found that a website's content addressing compensation and organizational culture, as well as the website's navigational usability were positively related to perceptions of attraction by job seekers. Similarly, Allen et al. (2004) demonstrated that both the amount of organization information and the amount of 
job information available on an organizational website were positively related to job seekers' intentions to pursue employment there. A study by Zusman and Landis (2002) showed that organizations who presented web pages of greater attractiveness were preferred to those presenting web pages of lesser attractiveness.

More recently, Williamson et al. (2010) examined how firm attributes, specifically prestige, influence the effectiveness of recruitment websites. Results showed that website attributes relating to website vividness (i.e., the extent to which a Web site uses images and/or sounds to enhance users' sensory experiences) were more effective in increasing applicant attraction for firms with higher prestige, whereas instrumental attributes (i.e., amount of company and job information provided on website) were more effective in increasing applicant attraction for firms with lower prestige. As such, it is necessary to realize that an organization's prestige level may influence the usefulness of their web-based recruitment tactics.

\section{Current Study}

Although preliminary evidence linking organizational website characteristics with organizational attraction continues to accumulate (e.g., Breaugh, 2008), scholars have called for a need to examine the mechanisms through which these characteristics lead to increased attraction (Ployhart, 2006). In other words, how exactly are applicants interpreting the information on web-based recruitment materials in a way that leads to increased attraction to the organization? A key difference in the shift from traditional recruitment methods to web-based methods is that organizations are able to provide much more information about the organization on these new platforms. This additional space, 
and flexibility in how they design the recruitment materials has introduced new features such as employee testimonials, benefits information, organizational value statements, and general information about daily life working at the organization through pictures and detailed descriptions. Organizations provide this increased information in hopes that job seekers favorably view the organization as a potential employer (Braddy et al., 2006). In the process of viewing recruitment materials, potential applicants are able to draw inferences about the organization, such as perceptions of the organizational image. As previously discussed, perceptions of image directly affect how potential applicants view the organization, through processes such as perceived P-O fit, image congruity beliefs, and self-continuity, which in turn impact how attracted and likely potential applicants are to pursue employment with the organization.

A central goal of this research is to identify aspects of web-based recruitment media that influence viewer perceptions of image, specifically operationalized as organizational personality. Previous research in this domain has focused solely on image perceptions relating to recruitment material on corporate websites. Given the increased prevalence of organizations embracing SNW sites as a way to communicate with members external to the organization, the study will examine aspects of corporate websites that impact perceptions of image as well as aspects of SNW profiles, specifically Facebook, that impact perceptions of image. Additionally, due to the recent introduction of managing image perceptions on web-based media, particularly through social networking sites, this study examines the potential differences in image projections through these two mediums. Lastly, in an effort to provide practitioners with advice on how to best manage their image projections on web-based recruitment media, we assess 
the relative impact of each website feature on the various dimensions of organizational image. The following section reviews specific literature and relevant theories which shape the research goals of this study.

\section{Website Design and Perceptions of Image}

As discussed above, previous research has found that job seekers use the information provided on organizational recruitment websites (e.g., pictures, employee testimonials) to determine overall fit and attraction to the hiring organization based on perceptions of image (Braddy et al., 2009; Dineen et al., 2002; Kroustalis, 2006). We now examine the theoretical and empirical research suggesting how these image perceptions are formed through web-based recruitment media.

\section{Signaling Theory}

Although it originally stems from economics research on the role of information possessed by a buyer and a seller (Spence, 1974), signaling theory has been adopted to explain the interaction between potential applicants and recruiting organizations (Rynes, Bretz, \& Gerhart, 1991; Rynes \& Miller, 1983; Spence, 1973). Signaling theory suggests that in the face of incomplete information about an organization, individuals will call on whatever information is available to make inferences about unknown job and organizational attributes (Rynes, 1991; Spence, 1973). Support for signaling theory, in the recruitment context, has been found as applicants have been shown to use perceptions of recruiters to shape their impressions of hiring organizations (Goltz \& Giannantonia, 1995; Rynes at al., 1991; Rynes \& Miller, 1983; Turban, 2001; Turban et al., 1998). For instance, if a recruiter is perceived as both competitive and creative, applicants may 
assume these are characteristics of the entire organization as well. Rynes and Miller (1983) argue that this likely occurs because applicants view recruiters as being representative of their respective organizations. It is important to highlight that recruiting experiences can have signaling value under many circumstances, but more so in situations where prospective applicants have limited knowledge of the organization (Rynes et al., 1991) — a trend that is becoming increasingly more prevalent with webbased recruitment methods.

Since job seekers often have limited knowledge of organizations prior to beginning the selection process, recruitment material is likely their primary source of information about the hiring company (Rynes \& Miller, 1983). Based on this, features of recruitment material that may not appear to have a direct connection to the job or organization (e.g., pictures, layout) can become cues or signals for perceptions of image of the organization (Rynes et al., 1991; Turban, 2001; Turban et al., 1980). In line with other research on this topic (e.g., Braddy et al., 2006, Braddy et al., 2009), this study employs the principles of signaling theory to explain how potential applicants form organizational image perceptions after viewing web-based recruitment media.

\section{Websites and Perceptions of Organizational Culture}

Two studies have been instrumental in guiding the research on the effects of website content features on applicant perceptions of organizational image. In 2006, Braddy et al., conducted a qualitative study to identify aspects of recruitment websites that influenced job seekers' perceptions of organizational culture. Nine organizational culture dimensions were examined, including innovation, emphasis on rewards, 
supportiveness, outcome-orientation, attention-to-detail, team-orientation, aggressiveness, decisiveness (O'Reilly, Chatman, and Caldwell, 1991), and diversity (Braddy et al., 2006). More specifically, they examined the impact of website design features, website content, and organizational policy on each of the nine organizational culture dimensions for select Fortune 500 companies. Results showed that both website features and content pertaining to organizational values, policies, awards, and goals affected viewers' perceptions of organizational culture. For example, the Innovation dimension was found to be related to components such as pictures of innovative products and advanced production facilities, employee testimonials mentioning innovation, awards won for innovation, sophisticated language choice, colorful and attractive web page design, and a focus on technology (see Braddy et al., 2006 for detailed results). A more detailed description of specific website features and content found to be related to each dimension, particularly for those highly related to image, will be discussed below.

Building on the qualitative results gathered in the previous study, Braddy et al. (2009) conducted a second study to empirically examine the relationship between website features and perceptions of culture. Through experimental manipulation of select features (e.g., pictures, testimonials, organizational policies, and awards won), they examined viewer's perceptions of the nine organizational culture dimensions. Results generally showed that website features and content could effectively be used to convey organizational culture attributes as predicted. More so, congruence between organizational culture perceptions and individual culture preference was found to positively impact perceptions of fit and organizational attraction (Braddy et al., 2009) 
Given the significant overlap of these organizational culture dimensions (Braddy, 2009; O'Reilly et al., 1991) with Slaughter et al.'s (2004) dimensions of organizational personality, we believe these findings are directly applicable to perceptions of image as operationalized in this study.

\section{Websites and Perceptions of Individual Personality}

Parallel to research on website components as indicators of organizational image, there is a growing body of literature interested in assessing individual personality through user profiles on social networking sites (e.g., Facebook, Twitter) (Havenstein, 2008). Based largely on the same philosophies as those discussed above, scholars and employers are exploring the $\mathrm{Web}$ as a means of gathering information about current and future employees. Many of these studies examine website components such as written content, pictures, layout, music, videos, number of friends, and the frequency and nature of interactions with others (Amichai, Humburger, \& Vinitzky, 2010; Karl, Peluchette, \& Schlaegel, 2010; Kluemper et al., 2012).

Kluemper et al. (2012), for example, examined the relationship of self-ratings of personality compared to other-ratings of personality which had been assessed based on content provided on Facebook profiles pages. Results not only showed that the two ratings were significantly related to each other, but other-ratings based on SNW profiles were more strongly related to job performance ratings than self-ratings. Back et al. (2012) also support for correlations between self and other-ratings based on personality perceptions from Facebook profiles, for all personality dimensions except Neuroticism. 
Going a step further than other-ratings of personality, Sumner, Bryers, and Shearing (2011) examined the extent to which personality traits could be measured based solely on Facebook usage, activities, and language use. Specifically, they gathered 79 Facebook data points (e.g., number of friends, number of photo albums, sex, age, number of profile pictures, etc.) and examined their ability to predict self-reported personality ratings. Results showed a number of significant relationships between their Facebook activity and personality traits. For example, Extraversion was positively related to number of friends, number of photo albums, number of profile pictures, and number of comments posted, and negatively related to number of books listed. Conversely, Openness to Experience was positively related to biography length, quotes length, number of photos, and number of books, movies and music.

Similarly, Quercia, Kosinski, Stillwell and Crowcroft (2011) examined the relationship between Twitter user activity and the Big Five personality dimensions. Findings showed support for being able to predict individual personality based on three features of the individual's twitter account, specifically the number of other users they follow, the number of followers they have, and the number of time they have been listed on other users' reading lists. Specifically, they found that popular (i.e., high follow count and high followers count) and influential users were related to high levels of Extraversion and low levels of Neuroticism, popular users were also related to high levels of Openness to Experience, and lastly, influential users were related to high levels of Conscientiousness. 
Although continued research in this domain is warranted, some of the same methods employed for assessing individual personality from user profiles can be useful for assessing organizational image from web-based recruitment media. Additionally, since the organizational personality framework can be viewed as an organization-specific counterpart of the Big Five (Slaughter et al., 2004; 2007), website components shown to be related to individual personality dimensions offer insight into measuring the complementary image dimensions.

\section{Research Questions and Hypotheses}

This section lists research questions and hypotheses for this study. Specifically, we discuss: (1) research questions relating to the identification of objective website indicators for image, as operationalized by organizational personality, (2) hypotheses relating to the congruence between perceptions of image and objective assessments of image, (3) hypotheses relating to the divergence of image across recruitment media, and (4) research questions pertaining to identifying which objective indicators are most influential for specific image dimensions.

\section{Identifying Objective Indicators}

According to Slaughter et al. (2004), the Boy Scout dimension is characterized by organizations that are friendly, attentive to people, family-oriented, helpful, clean, and honest. The conceptualization is in-line with Braddy et al.'s (2006) supportiveness and team-orientation dimensions, both of which promote helpfulness, sharing, and cooperation. The supportiveness dimension has shown to be related to aspects such as pictures of teamwork, employee testimonials highlighting support, good benefits, 
continuing education programs, discussion forums, valuing diversity, and references to trust, respect and sharing of information. Similarly, the Team-Orientation dimension has been linked to features such as pictures of people working together, employee testimonials emphasizing teamwork, special subsection of website devoted to teamwork, geographical dispersion, community involvement, emphasizing team-based approach to working, and valuing diversity (Braddy et al., 2009).

The Boy Scout dimension is also viewed as being complementary to Agreeableness because of the shared friendliness, cooperation, and people-oriented components (Slaughter et al., 2004; Slaughter \& Hreguras, 2009), as well as Conscientiousness since being cooperative and friendly facilitates accomplishing more (Le Pine \& Van Dyne, 2001). Together, these Big Five dimensions have been linked to personal website features relating to maturity, higher website activity, more connections to others, and more pictures (Kluemper, et al., 2012; Querciaet al., 2011; Sumner, et al., 2011).

Research Question 1 a

Will site visitors, awards for best places to work, number of photos of people, employee recognition, frequency word 'support', discussion forum, frequency of word 'diversity', contact information, benefits listed, continuing education information, environmental-awareness, community involvement, frequency of word 'trust', and the frequency of word 'respect' predict the Boy Scout dimension for corporate websites? 


\section{Research Question $1 b$}

Will page likes, awards for best places to work, number photos of people, frequency of posts, fan recognition, frequency of word 'support' posted, interactive posts, frequency of word 'diversity', contact information, benefits listed, continuing education information, environmental-awareness posts, community involvement posts, frequency of word 'trust', and the frequency of word 'respect' predict the Boy Scout dimension for company Facebook profiles?

The Innovation dimension is indicative of organizations that are perceived as being interesting, exciting, unique, and creative (Slaughter et al., 2004). This dimension shares clear similarities with Braddy et al.'s (2006) Innovation dimension. Additionally, diversity can be seen as a way of achieving creativity, by embracing a diverse range of backgrounds and ideas. Previous literature has linked the Innovation dimension to components such as pictures of innovative products and advanced production facilities, portion of the webpage devoted to innovation, employee testimonials mentioning innovation, awards won for innovation, large number of products or services under development, sophisticated language choice, colorful and attractive web page design, focus on technology, valuing education, and encouraging risk taking. Alternatively, the diversity dimension has been linked to features such as pictures of diverse employees working, testimonials from a diverse set of employees, statistics on minority employment, specific subset of webpage devoted to diversity, mentioning diversity events or trainings, diversity/minority employment awards, valuing creativity in the 
workplace, and specific mentions of being an EEO employer, global community, and diverse workplace (Braddy et al., 2009).

Openness to Experience has been described as the complementary individual personality trait since individuals high on this dimension are generally imaginative, curious, cultured, and intellectual (Slaughter et al, 2004; Slaughter \& Hreguras, 2009). In personal website research, this dimension has been linked to content length (both bio and postings), books, interests listed, movies, music, and number of posts and pictures (Kluemper, et al., 2012; Querciaet al., 2011; Sumner, et al., 2011).

Research Question $2 a$

Will training/education opportunities, advertising new product/service launches, frequency of word 'innovation', frequency of word 'technology', frequency of word 'risk', links to other social media, variety of color in text, language choice in main page, use of flash or video, links within careers section, about us length, contest or survey for viewers, discussion forum, and diversity initiatives predict the Innovation dimension for corporate websites?

Research Question $2 b$

Will training/education opportunities, advertising new product/service launches, frequency of word 'innovation', frequency of word 'technology', frequency of word 'risk', links to other social media, variety of color in text, language choice in about us, video posts, links within profile, about us length, games or contests for viewers, interactive posts, and diversity initiatives predict the Innovation dimension for company Facebook profiles? 
The Dominance dimension encapsulates organizations that are viewed as successful, popular, dominant, busy, and active (Slaughter et al., 2004). The dominant and busy aspects of the dimension highlight conceptual similarities with Braddy et al.'s (2006) Aggressiveness dimension and Outcome-Orientation dimensions. The Aggressiveness dimension is shown to be related to aspects such as pictures of people working, pay for performance, organizational awards won, general aggressiveness of recruiting webpage, size of organization, plans for expansion, including sales or production figures on website, and references to winning, competition and cutting-edge. Similarly, the Outcome-Orientation is linked to components such as employee testimonials attesting to advancement opportunities, extremely professional looking webpages, use of diagrams of flow charts, inclusion of financial report data, bonus systems, organizational performance awards, and specific references to success, winning, high quality, high standards, and goals (Braddy et al., 2009).

In terms of personality traits, the rigid and sometimes fearful components of Neuroticism, and the sociable, bold, and active components of Extraversion, highlight conceptual overlap with the Dominance dimension (Slaughter et al, 2004; Slaughter \& Hreguras, 2009). In personal websites, these traits have been linked to frequency of postings and number of friends, photo albums and negatively associated with information about reading material (Kluemper, et al., 2012; Querciaet al., 2011; Sumner, et al., 2011).

Research Question 3a:

Will performance awards listed, financial information provided, use of flow chart/diagram, bonus-system listed, frequency of word 'success', media articles 
listed, links within careers section, language availability of webpage, site traffic counter, frequency of word 'winning', community involvement, events listed, and charity information predict the Dominance dimension for corporate websites?

Research Question $3 b$

Will performance awards listed, financial information provided, use of flow chart/diagram, bonus-system listed, frequency of word 'success', media articles posted, links within profile, page likes, people talking about page, frequency of word 'winning', community involvement posts, events listed, charity information, and the frequency of posts predict the Dominance dimension for company Facebook profiles?

Organizations that are perceived as being Thrifty are perceived as being low budget, poor, low class, simple, deprived, and sloppy. Although none of Slaughter et al.'s (2004) dimensions share conceptual similarity with Thrift, Attention to Detail can be viewed as the counterpart of the 'sloppy' component of this dimension. Accordingly, features such as detailed job descriptions and employee testimonials, focus on safety, fact based decision making and specific references to detail-orientation and being analytical, which have been linked to Attention to Detail, would be expected to show low levels of Thrift (Braddy et al., 2009).

Likewise, individuals low on Conscientiousness and Extraversion have been described as the counterpart of this image dimension, whereas Agreeableness is seen as complementary (Slaughter et al, 2004; Slaughter \& Hreguras, 2009). Based on this, corporate website components related to frequency of postings and number of friend, 
photo albums, and age would be influential components for Thriftiness (Kluemper, et al., 2012; Querciaet al., 2011; Sumner, et al., 2011).

\section{Research Question $4 a$}

Will the number of images/photos, use of flash or video, frequency of word 'budget', amount of text, links within careers section, links to other social media, spelling mistakes, and the frequency of word 'safety' predict the Thrift dimension for corporate websites?

Research Question $4 b$

Will the number of images/photos, video posts, frequency of word 'budget', amount of text in 'about us', links within profile, links to other social media, frequency of posts, spelling mistakes, and the frequency of word 'safety' predict the Thrift dimension for company Facebook profiles?

Lastly, the Style dimension is characterized by organizations that are perceived as stylish, fashionably, trendy, and hip (Slaughter et al, 2004). This dimension shares some conceptual overlap with the Innovation dimension (Braddy et al., 2006), such as taking risks and sophistication. Additionally, Style can be viewed as complementary to the Openness to Experience personality trait through its intellectual, snobbish, and creative similarities (Slaughter et al, 2004; Slaughter \& Hreguras, 2009). Consequently, website components relating to these dimensions are expected to be related. 


\section{Research Question 5 a}

Will the number of photos, amount of graphics/art, use of flash or video, music on site, variety of color in text, links to other social media, events listed, celebrity or athlete endorsements, contest or survey for viewers, discussion forum, and diversity initiatives predict the Style dimension for corporate websites?

Research Question $5 b$

Will the number of photos, amount of graphics/art, video posts, music/audio posts, variety of color in text, links to other social media, events listed, celebrity or athlete posts, games or contests for viewers, interactive posts, and diversity initiatives predict the Style dimension for company Facebook profiles?

The objective indicators listed for Research Questions 1-5 are also summarized in Appendix A.

\section{Congruence between Subjective and Objective Image}

We argue that objective indicators for each dimension can be examined together to assess an organization's overall strength in each of the five image dimensions. Furthermore, we believe that objective measure of image will be representative of viewer perception of image when exposed to the same material. Specifically, we posit that:

H1. Perceptions of image based on exposure to corporate websites will converge with the image projections based on objective components of the corporate website 
H2. Perceptions of image based on exposure to Facebook profiles will converge with the image projections based on objective components of the Facebook profiles

\section{Divergence across Sources of Image}

Given the substantial amount of research linking perceptions of P-O fit to increased applicant attraction (e.g. Judge \& Cable, 1997), it is essential for organizations to effectively manage the image they are projecting through their recruitment sources so as to maximize the likelihood of attracting the best applicants. As such, we seek to examine how image projections vary based on exposure recruitment media, namely corporate websites and organization Facebook profiles.

According to media richness theory (MRT), communication outcomes depend on the match between media capacities and communication requirements (Daft \& Lengel, 1986). Furthermore, MRT posits that visual images, symbols, sounds, or information of a personal nature often require media with a greater capacity. Recruitment, for example, requires both a personal connection and communication of ambiguous information (e.g., values, culture, etc.) in order to effectively persuade potential applicants to consider joining the organization. Researchers have found evidence that communication media differ in terms of their effectiveness in communicating certain types (Allen et al., 2004). Based on MRT, complex and ambiguous information, such as organizational image, would be better relayed in more sophisticated recruitment methods that allow for richer message transmittal. Therefore, because different recruitment media are limited in message richness relaying capacity, perceptions of image and objective image indicators 
will vary based on recruitment media. Lastly, since research shows recruitment messages impact subsequent applicant attitudes and decisions (e.g., Rynes at al., 1991), image perceptions after exposure to any recruitment media at all, would alter pre-existing image perceptions.

H3. Perceptions of Organizational Personality will vary across different recruitment sources (no exposure, website, Facebook) for each dimension

H4. Organizational Personality objective scores will vary across different recruitment sources (website, Facebook) for each dimension

\section{Relative Predictive Validity of Objective Indicators}

Lastly, this study also seeks to provide information for practitioners interested in developing or modifying their image projections through-web based recruitment methods as part of their broader human resource management strategy. Specifically, this research aims to identify practical insights into how organizations can best design their recruitment websites, both corporate website and Facebook profile, based on the image profile they are trying to project to job seekers. By effectively managing organizational image perceptions from web-based recruitment efforts, companies will maximize their chance of attracting qualified applicants, thus producing the largest returns from investment in Web recruitment media.

As such, a final goal of this study will be to identify which website features are most indicative of a particular image projection as operationalized by Slaughter's organizational personality dimensions (e.g., Boy Scout, Thrift, Style, etc.). In other words, for each recruitment medium (i.e., corporate website and Facebook profile), what 
features are most related to each of the five organizational personality dimensions? For example, within the set of corporate website features that were found to be related to the Boy Scout dimension, which features are most related to perceptions of Boy Scout? Specifically, this research seeks to pursue the following supplementary analyses:

Research Question 6: Identify the related contribution of the final corporate website indicators on each of the perceptions of organizational personality dimensions

Research Question7: Identify the related contribution of the final Facebook profile indicators on each of the perceptions of organizational personality dimensions 


\section{Chapter III: Methodology}

In Chapter III the methodology that was used to test the hypotheses presented in Chapter II is described. The company database used for this study was compiled in two phases. Phase one of data collection consisted of a student sample to establish perceptions of organizational personality for each company specific for each condition (after exposure to corporate website, after exposure to Facebook company profile, and no exposure to recruitment material). Phase two of data collection contributed to the database created in phase one by adding researcher-ratings of organizational personality using objective indicators specific to each recruitment source (corporate Website and Facebook profile). Details of each phase are organized in the following order: company database overview, phase one procedures and measures, and phase two procedures and measures.

\section{Company Database}

Fortune Magazine's 2012 top 500 companies were used as a starting point to select the list of companies to be included in the study. In order to be selected for the study, the company was required to have both a corporate website with a 'careers' page and an active company Facebook profile (i.e., company initiated activity within the past 30 days). The principal investigator reviewed the list in descending order beginning with number 500 and selected companies for inclusion until reaching the target sample size of 102 companies. Appendix B provides the final set of companies included in the study. For phase one of this study, a student sample was used to generate perceptions of organizational personality for each of the 102 companies in the database. For phase two, 
the principle investigator added to the database by manually coded objective ratings taken from each company's corporate website and Facebook profile. A graphical overview of the components of the company database is provided in Appendix C.

\section{Phase One: Perceptions of Organizational Personality}

\section{Phase One Procedures}

The purpose of phase one was to establish general perceptions of organizational personality for each of the 102 companies in the database. Furthermore, each company's organizational personality was assessed in each of the following three conditions:

exposure to corporate Website, exposure to Facebook company profile, and No Exposure to recruitment material.

Data from a sample of students was collected from Florida International University through an online psychology research pool. In order to participate in the study, students were required to have a working Facebook account or to have operated one within the past 12 months. This requirement was implemented to ensure that all participants were comfortable and familiar with the layout of Facebook in case they were selected for the Facebook condition. Participants were asked to participate in an online study about organizational personality. After agreeing to participate, students were randomly assigned to one of three experimental conditions: primed with exposure to the company website 'careers' page (i.e., employment opportunities page), primed with exposure to the company Facebook profile, or not exposed to any recruitment material. Once assigned to a condition, the survey administration program randomly chose three companies from the company database (See Appendix B) for each participant to rate. If a 
participant was in the No Exposure condition and was not familiar with a company, the participant was presented with another company to rate. Appendix D provides a graphical representation of the online survey used to collect Phase 1 data,

The survey for the three experimental conditions contained the same set of items. However, participants in the Website and Facebook condition were instructed to review some company materials prior to being presented with the items. Those in the Website condition were presented with a series of screen shots of the 'careers' homepage taken directly from the target company's corporate website. Similarly, participants in the Facebook condition were presented with a series of screenshots taken directly from the target company's Facebook profile showing activity in the past 30 days. After reviewing the screenshots, participants were presented with questions relating their perceptions of prestige of the company (organizational reputation scale) and their impression of the company (organizational personality scale). The screenshots were standardized across companies within each condition. For the Website condition, screen shots were taken of the main 'careers' page and the 'about us' page (i.e., who we are) within the careers section. For the Facebook condition, screen shots were taken of the profile homepage showing only 'posts by page' (i.e., posts by the company and not posts by other Facebook users on the company timeline) and was limited to the past 30 days of activity, as well as screenshots of the 'about us' section of the profile. Additionally, in order to ensure that screenshots reflected the target company at the same point in time, website and Facebook screenshots were captured within 24 hours of each other. Alternatively, participants in the No Exposure condition were not exposed to any recruitment material before being presented with the prestige and organizational personality items on the web-based survey. 
As mentioned above, participants in the No Exposure condition who had no familiarity with a company were assigned an alternate company to rate. Accuracy test items were included in each of the experimental conditions to minimize the impact of random responses.

Organizational personality scores were computed for each of the five OP dimensions (e.g., Dominance, Thriftiness, etc.) for each individual rater. Next, each company's OP score for each dimension was computed by averaging the scores of the four (or more) participants assigned to that particular company. This was performed three times for each experimental condition (Website, Facebook, and No Exposure). These aggregated agreement scores reflect general applicant perceptions for each company and were used in all subsequent analyses.

\section{Phase One Measures}

Name Recognition. Participants in the No Exposure condition were asked if they recognized the name of the company they were being asked to rate. Response options were 'yes' or 'no.' Participants who indicated they did not recognize the name of the company were randomly assigned to another company.

Prestige. Participants in each experimental condition were asked to rate the firm's reputation as an employer. In line with previous studies on this topic (e.g., Williamson et al., 2010), prestige was assessed using four items adapted from Turban et al. (1998). Participants were asked the extent to which they agreed that (1) most graduates are interested in this firm as an employer, (2) this company has a reputation of being an excellent employer, (3) this company has an excellent reputation on campus, (4) 
I have heard a lot of good things about this firm. Response options were on a 7-point scale of agreement $(1=$ Strongly Disagree to $7=$ Strongly Agree $)$. The coefficient alphas for this study were .83 for the Website and Facebook conditions, and .79 for the No Exposure condition. Coefficient alphas were computed at the individual level prior to aggregating any data.

Self-Report Organizational Personality. Organizational personality was measured using Slaughter et al.'s (2004) 33-item organizational personality scale. The scale is designed to measure five different dimensions: Boy Scout $(n=9)$, Innovation ( $=7)$, Dominance $(n=5)$, Thriftiness $(n=8)$, and Stylishness $(n=4)$. Participants were asked to rate the extent to which each of the 33 trait adjectives described the organization that they were assigned. Response options were on a 5-point scale of agreement $(1=$ Strongly Disagree to 5 = Strongly Agree). Items include, "Cooperative, Friendly, Low class, etc." A higher score on each dimension indicates a higher display of those characteristics. Two of the items were negatively worded and required reverse-coding. The coefficient alpha reliabilities for the Website condition ranged from $\alpha=.86-.96$ (Boy Scout $\alpha=.94$, Innovation $\alpha=.90$, Dominance $\alpha=.86$, Thriftiness $\alpha=.92$, Stylishness $\alpha=.96)$. For the Facebook condition, the coefficient alpha reliabilities ranged from $\alpha=.80-.95$ (Boy Scout $\alpha=.91$, Innovation $\alpha=.89$, Dominance $\alpha=.80$, Thriftiness $\alpha=.91$, Stylishness $\alpha=.95$ ). Lastly, the coefficient alpha reliabilities for the No Exposure condition, ranged from $\alpha=.78-.94$ (Boy Scout $\alpha=.89$, Innovation $\alpha=.84$, Dominance $\alpha=.78$, Thriftiness $\alpha=.91$, Stylishness $\alpha=.94)$. Coefficient alphas were computed at the individual level prior to aggregating any data. 
See Appendix F for the full measure.

Demographics. Participants in phase one were asked to provide background information at the beginning of the survey. Demographic items gathered information relating to: gender, ethnicity, age, marital status, and employment details.

Test Items. Three test items for each experimental condition were dispersed throughout the survey. These items helped the principal investigator determine if participants were paying attention while responding to the survey. Incorrect responses to these items resulted in elimination of the data line from the set prior to analyses. An example of a test item in the survey is "For this question, please select the response Strongly Disagree".

\section{Phase Two: Developing Objective Ratings of Organizational Personality Phase Two Procedures}

The second data collection phase was focused on developing objective ratings of organizational personality for each company through the use of objective indicators. Whereas phase one focused on capturing perceptions of OP, this phase focused on developing objective indicators of OP. For this phase, the No Exposure condition was not used since there was no recruitment source to examine. For the other two conditions, objective indicators of OP were examined for each recruitment source (i.e., corporate Website, Facebook profile). More specifically, indicators of organizational personality were examined and then scored to create objective ratings of each of the five OP dimensions. The development of the objective OP ratings was based on both content validation and empirical validation techniques. 
The objective OP ratings were developed using a three-step approach as follows: 1) collect ratings for full list of Website and Facebook profile OP indicators for each company; 2) perform content validation of indicators through a Subject Matter Expert (SME) Sort Task to revise classification; and 3) perform an empirical validation using structural equation modeling to further revise and finalize classification. Each of these steps is described in detail below. The indicators that were retained from step one to step two represent the final set of indicators predicting each OP dimension for subsequent hypotheses testing.

Step One: Indicator Ratings. The principal investigator began with a list of rationally and theoretically derived indicators for each OP dimension specific to the corporate websites and Facebook profiles as summarized in research questions 1-5. Each indicator was classified into one or more of the OP dimensions, such that each OP dimension was represented by a rationally determined set of indicators. Examples of website indicators include number of site visitors, number of pictures, use of flash in homepage (yes/no), etc. Examples of Facebook profile indicators include number of page 'likes', number of pictures posted, use of game/contest on profile (yes/no) (see Appendix A for complete list for each OP dimension). Detailed descriptions of each indicator and respective scoring instructions are discussed in the following section.

The principal investigator then used the same screenshots used for the self-ratings of OP in phase 1 to objectively score OP for each company. First, the principal investigator scored each objective indicator using the corporate website screenshots for 
the company. Then, the process was repeated to rate the objective indicators of the Facebook profile using the Facebook profile screenshots.

Step Two: Content Validation. After completing the objective evaluations of the website and Facebook profile, the classification of each OP indicator was content validated through an SME Sort Task. More specifically, a group of 14 Subject Matter Experts (SMEs) were chosen to participate in a sort validation task. The SMEs were randomly assigned to validate the two sets of indicators, seven evaluated the website indicators and seven evaluated the Facebook indicators. Subject Matter Experts who were selected to participate in the Facebook indicators sort task were be required to have an active Facebook profile (at the time of the exercise or within the past 12 months). Each SME received a detailed description and definition of the five organizational personality dimensions as defined by Slaughter et al. (2004). A sample of the descriptive information for the Website sort group is provided in Appendix G. The Facebook SMEs were provided with similar information which was only modified for Facebook profile references. After all SMEs acknowledged a clear understanding of the parameters of each dimension, they were provided with a master list of all indicators for their medium (i.e., Website, Facebook) through an online survey. Subject Matter Experts then evaluated each indicator separately to determine which dimension(s), if any, each particular indicator represented. See Appendix F for a graphical representation of the task. SMEs were asked to perform this task completely independently in order to minimize inter-rater bias. 
Subject Matter Expert ratings were then compiled to reflect the total number of people that selected each dimension for the target indicator. A minimum of five SMEs (or $71 \%$ ) needed to have selected a dimension in order for the indicator to be retained as representing that particular dimension. For example, a minimum of five individuals needed to indicate that "number of page visitors' indicates the Dominance dimension in order for that indicator to reflect Dominance. Additionally, if $71 \%$ agreement was achieved, an indicator was classified as representing a particular OP dimension, even if the item was not previously classified to that dimension. It is possible that indicators reflect more than one dimension. This revised version of the indicators was used for the empirical validation in the following step.

Step Three: Empirical Validation. The final step entailed an empirical validation of the objective OP indicator classification, as supported by the content validation, through structural equation modeling. To empirically test the factor structure of organizational personality for Website and Facebook indicators, two separate Confirmatory Factor Analyses (CFA) were run using AMOS, once for the Website indicators and once for the Facebook indicators. This analysis determined how well the proposed factor structure of the indicators fit the data. In order to establish that a fivefactor model is supported, two models were run and compared to one another to determine which has the better model fit. The first model was a uni-factor model (i.e., all indicators for every OP dimension loading on a latent variable) and the second model was a five-factor model (i.e., all indicators loading on each respective dimension as classified by the content validation). Several tests and indices were used to determine the model fit, including chi-square, the comparative fit index and the root mean square approximation. 
A chi-square difference test was used to determine whether the uni-factor or five-factor model is a better representation of the data. This test is appropriate when comparing a model (i.e., the uni-factor model) that is nested within another model (i.e., the five-factor model) and is performed by subtracting the smaller chi square and its degrees of freedom from the larger chi square and degrees of freedom.

If good model fit was achieved for the five-factor structure, a more detailed examination of each OP dimension was performed by reviewing factor loadings for each indicator. Indicators with low factor loadings were further examined for possible deletion. Once good model fit was achieved for each dimension, a composite score was calculated for each dimension comprised of all of the indicators remaining in the final model. This final refined score, as modified by both the content validation and the empirical validation, served as the objective OP rating for each organization.

\section{Measures for Phase Two}

Website Indicators of Organizational Personality. Organizational personality as reflected by a company's corporate website was measured using webpage-specific indicators that reflect Slaughter et al.'s (2004) organizational personality framework. The indicators are designed to measure specific characteristics of a web page that are indicative of at least one of the five dimensions. Specific website indicators are listed below.

- Boy Scout: site visitors (\#), awards for best places to work (\#), number of photos of people (\#), employee recognition $(\mathrm{y} / \mathrm{n})$, frequency word 'support'

(\#), discussion forum $(\mathrm{y} / \mathrm{n})$, frequency of word 'diversity' (\#), contact 
information $(\mathrm{y} / \mathrm{n})$, benefits listed (\#), continuing education information $(\mathrm{y} / \mathrm{n})$, environmental-awareness $(\mathrm{y} / \mathrm{n})$, community involvement $(\mathrm{y} / \mathrm{n})$, frequency of word 'trust' (\#), frequency of word 'respect' (\#)

- Innovation: Training/education opportunities $(\mathrm{y} / \mathrm{n})$, advertising new product/service launches $(\mathrm{y} / \mathrm{n})$, frequency of word 'innovation' (\#), frequency of word 'technology' (\#), frequency of word 'risk' (\#), links to other social media (\#), variety of color in text (\#), use of flash or video (y/n), links within careers section (\#), about us length (\# words), contest or survey for viewers $(\mathrm{y} / \mathrm{n})$, discussion forum $(\mathrm{y} / \mathrm{n})$, diversity initiatives $(\mathrm{y} / \mathrm{n})$

- Dominance: performance awards listed (\#), financial information provided $(y / n)$, use of flow chart/diagram $(y / n)$, bonus-system listed $(y / n)$, frequency of word 'success' (\#), media articles listed (\#), links within careers section (\#), language availability of webpage (\#), site traffic counter (\#), frequency of word 'winning' (\#), community involvement (y/n), events listed (y/n), charity information $(\mathrm{y} / \mathrm{n})$

- Thrift: number of images/photos (\#) (reverse scored), use of flash or video $(y / n)$ (reverse scored), frequency of word 'budget' (\#), amount of text (\#)(reverse scored), links within careers section (\#) (reverse scored), links to other social media (\#) (reverse scored), spelling mistakes (\#), frequency of word 'safety' (\#)(reverse scored)

- Style: number of photos (\#), amount of graphics/art (\#), use of flash or video $(y / n)$, music on site $(y / n)$, variety of color in text (\#), links to other social 
media (\#), events listed $(\mathrm{y} / \mathrm{n})$, celebrity or athlete endorsements $(\mathrm{y} / \mathrm{n})$, contest or survey for viewers $(\mathrm{y} / \mathrm{n})$, discussion forum $(\mathrm{y} / \mathrm{n})$, diversity initiatives $(\mathrm{y} / \mathrm{n})$

\section{Facebook Profile Indicators of Organizational Personality. Organizational}

personality as reflected by a company’s Facebook profile was measured using profilespecific indicators that reflect Slaughter et al.'s (2004) organizational personality framework. The indicators are designed to measures specific characteristics of a Facebook profile that are indicative of at least one of the five dimensions. Specific Facebook indicators are listed below.

- Boy Scout: page likes (\#), awards for best places to work (\#), number of photos of people (\#), frequency of posts (\# per week), fan recognition (y/n), frequency word 'support' posted (\#), interactive posts (\#), frequency of word 'diversity’ (\#), contact information (y/n), benefits listed (\#), continuing education information $(\mathrm{y} / \mathrm{n})$, environmental-awareness posts (\#), community involvement posts (\#), frequency of word 'trust' (\#), frequency of word 'respect' (\#)

- Innovation: training/education opportunities (y/n), advertising new product/service launches (\#), frequency of word 'innovation' (\#), frequency of word 'technology' (\#), frequency of word 'risk' (\#), links to other social media (\#), variety of color in text (\#), video posts (\#), links within profile (\#), about us length (\# words), games or contests for viewers (y/n), interactive posts(\#), diversity initiatives $(\mathrm{y} / \mathrm{n})$ 
- Dominance: performance awards listed (\#), financial information provided $(y / n)$, use of flow chart/diagram $(y / n)$, bonus-system listed $(y / n)$, frequency of word 'success' (\#), media articles posted (\#), links within profile (\#), page likes (\#), people talking about page (\#), frequency of word 'winning' (\#), community involvement posts (\#), events listed (\#), charity information (y/n), frequency of posts(\#/week)

- Thrift: number of images/photos (\#) (reverse scored), video posts (\#) (reverse scored), frequency of word 'budget' (\#), amount of text in 'about us' (\#) (reverse scored), links within profile (\#) (reverse scored), links to other social media (\#) (reverse scored), frequency of posts (\# per week) (reverse scored), spelling mistakes (\#), frequency of word 'safety' (\#)(reverse scored)

- Style: number of photos (\#), amount of graphics/art (\#), video posts (\#), music/audio posts (\#), variety of color in text (\#), links to other social media (\#), events listed (\#), celebrity or athlete posts (\#), games or contests for viewers $(\mathrm{y} / \mathrm{n})$, interactive posts(\#), diversity initiatives $(\mathrm{y} / \mathrm{n})$

\section{Plan of Analysis}

The first step included creating aggregate perceptions of organizational personality scores from the student data collected during phase one. Additionally, confirmatory factor analyses were run to determine the factor structure of perceptions of OP for each of the three experimental conditions of the phase one data. Phase two data was used to develop objective indicators of OP through indicator ratings, content validation, and empirical validations, ultimately answering research questions 1-5. 
Assessing convergence across measurement methods to test hypotheses one and two was tested using correlational analysis between perceptions of OP (from phase 1) and objective OP scores (from phase 2). The converegence analysis also served to establish external validation for the objective Website and Facebook OP scores. Source differentiation analyses to test hypotheses three and four included the following: a repeated-measures multivariate analysis of variance (MANOVA) to assess differences in perceptions of OP across the treatment conditions for the 5 dimensions; follow-up repeated-measures analyses of variance (ANOVA) to assess differences for each OP dimension separately; and correlational analysis between objective scores of OP between Website indicator scores and Facebook indicator scores. Lastly, supplementary indicator predictive validity analyses to address research question 6 includes the following: standard multiple regressions to measure the predictive validity of the final indicators on perceptions of organizational personality for each dimension. 


\section{Chapter IV: Results}

This chapter outlines the results obtained from phase one and two as well as subsequent analyses to test research questions and hypotheses. The analyses are grouped into five steps: creating aggregate perceptions of OP scores, development of objective indicators for OP, testing convergence between self-report and objective scores, source differentiation analyses across recruitment media, and objective indicator predictive validity. The next section describes in detail how these analyses were carried out.

\section{Creating Aggregate Perceptions of OP Scores}

\section{Student Sample}

Each of the 102 companies will be rated by a minimum of six students for each of the three experimental conditions - with each student rating three companies within the same condition. For the Website condition, a total of 949 ratings were collected across the 102 companies. Of these, 739 were retained after screening the accuracy items, for a response rate of $77.9 \%$. The final Website dataset included a minimum of four ratings for each of the 102 companies. For the Facebook condition, of the 821 initial ratings, 643 $(73.3 \%)$ were retained after accuracy screenings. Again, each company in the Facebook dataset was rated by a minimum of four raters. For the No Exposure condition, a total of 1,197 initial ratings were collected. However, only $385(32.2 \%)$ of these ratings had usable data beyond the company name recognition item (i.e., a majority of the students did not recognize the company assigned to them). In order to be consistent with the other experimental conditions and to have sufficient information to cancel out individual idiosyncrasies, only companies that had a minimum of 4 raters were retained for subsequent analyses. Of the 385 ratings, 302 (78.4\%) were retained after accuracy 
screenings. Appendix E provides a detailed list of the 50 companies retained in the No Exposure condition.

There were a total of 983 students across all three of the experimental conditions, of which $66.5 \%$ were female. Hispanics comprised a large portion of the sample (70.1\%), while 12.4\% were Black (non-Hispanic), 10.1\% White (non-Hispanic), 2.3\% Asian or Pacific Islander, 1.8\% Middle Eastern, and 3.3\% described themselves as "Other." The average age for the sample was 21 years old $(S D=4.55)$. Additionally, $94.4 \%$ of the students were single, $4.4 \%$ were married, and $1.2 \%$ were separated, widowed, or divorced. Almost half of the sample (39.6\%) was not employed. Of those that were employed, the half (50.2\%) worked between 20 and 40 hours, 39.4\% worked less than 20 hours, while the rest (10.4\%) worked 40 hours or more per week. The average tenure for those employed was 2.0 years $(S D=3.0)$. Mean comparison and chisquare analyses revealed no significant differences in demographic composition across the three experimental conditions.

\section{Preliminary Confirmatory Factor Analyses}

Structural equation modeling was employed to test the factor structure of perceptions of organizational personality for each experimental condition. Three separate Confirmatory Factor Analyses (CFAs) were run using AMOS 21.0 using a maximum likelihood algorithm to determine the factor structure of the items. This analysis determined how well the proposed factor structure (i.e., the five-factor structure for OP) fits the data. Two models were run and compared to one another to determine which had the better model fit. The first model was a uni-factor model (i.e., all 33 organizational personality items loading on a latent variable) and the second model was a five-factor 
model (i.e., the 33 items loading on each dimension separately) in which the latent variables were correlated with one another. As recommended by Kline (1998), regression imputation was used in SPSS to fill in the missing data prior to running the CFA models, since less than $10 \%$ of the data was missing.

As recommended by Bollen and Long (1993), Kline (2011), and Schumacker \& Lomax (2010), the global fit indices that were used to determine which model achieved a better fit are as follows: chi-square test of model fit, root-mean-square error of approximation (RMSEA), p-value for the test of close fit, the Tucker-Lewis index (TLI), comparative fit index (CFI), and standardized root mean square residual (Standardized RMR). Important to note, for models with sample sizes larger than 400, the chi-square test of model fit is almost always found to be statistically significant (denoting a bad model fit) (Kline, 2011). For this reason, a variety of other fit indices were also evaluated. The RMSEA measures the average fitted residual. A score of less than .1 (preferably less than .08) on this measure is indicative of a good model fit (Jaccard \& Wan, 1996). The CFI and TLI are indicative of better fit as their values approached 1 , and a score of .9 or better is indicative of a good model fit. A standardized root mean square residual value less than .08 was consistent with a good model fit (Hu \& Bentler, 1999). Additionally, a chi-square difference test was used to determine whether the unifactor or five-factor model was a better representation of the data. This analysis was completed three times, once for each experimental condition. Details for each experimental condition are presented below.

Website Condition CFA. The models were both statistically overidentified. A variety of indices of model fit were evaluated. For the one factor model, the overall chi 
square test of model fit was statistically significant, $\chi^{2}(496)=5123.45, p<.001$, the Root Mean Square Error of Approximation (RMSEA) was .11 and the $p$ value for the test of close fit was $p<.001$, providing an indicator of poor model fit. The Comparative Fit index was .79and the Tucker Lewis index was .76, indicating poor model fit. The SRMR was .42 , suggesting poor model fit. For the five factor model, the overall chi square test of model fit was statistically significant, $\chi^{2}(486)=4128.98, p<.001$, the Root Mean Square Error of Approximation (RMSEA) was .10, and the $p$ value for the test of close fit was $<.001$, indicating marginally good model fit. The Comparative Fit index was .83 and the Tucker Lewis index was .82, both indicators providing marginally adequate model fit. The SRMR was .07, suggesting good model fit. Importantly, the nested chi square test comparing this model to the uni-factor model yielded a statistically significant chi-square difference, $\chi^{2} \operatorname{diff}(10)=994.46, p<.001$, a result that suggests the five-factor model is a better fit. See Table for a summary for the Website CFA.

Facebook Condition CFA. The models were both statistically overidentified. A variety of indices of model fit were evaluated. For the one factor model, the overall chi square test of model fit was statistically significant, $\chi^{2}(496)=4578.57, p<.001$, the Root Mean Square Error of Approximation (RMSEA) was .11 and the $p$ value for the test of close fit was $p<.001$, providing an indicator of poor model fit. The Comparative Fit index was .75 and the Tucker Lewis index was .73, indicating poor model fit. The SRMR was .33, suggesting poor model fit. For the five factor model, the overall chi square test of model fit was statistically significant, $\chi^{2}(486)=3288.13, p<.001$, the Root Mean Square Error of Approximation (RMSEA) was .09, and the $p$ value for the test of close fit was $<.001$, indicating good model fit. The Comparative Fit index was .83 and 
the Tucker Lewis index was .81 , both indicating marginally adequate model fit. The SRMR was .07, suggesting good model fit. Importantly, the nested chi square test comparing this model to the uni-factor model yielded a statistically significant chi-square difference, $\chi^{2} \operatorname{diff}(10)=1290.45, p<.001$, a result that suggests the five-factor model is a better fit. See Table for a summary for the Website CFA.

No Exposure Condition CFA. The models were both statistically overidentified. A variety of indices of model fit were evaluated. For the one factor model, the overall chi square test of model fit was statistically significant, $\chi^{2}(496)=4707.41, p<0.001$, the Root Mean Square Error of Approximation (RMSEA) was .17 and the p value for the test of close fit was $p<.001$, providing an indicator of poor model fit. The Comparative Fit index was .38 and the Tucker Lewis index was .34, providing indicators of poor model fit. The SRMR was .14, suggesting poor model fit. For the five factor model, the overall chi square test of model fit was statistically significant $\chi^{2}(486)=1915.75, p<.001$, the Root Mean Square Error of Approximation (RMSEA) was .09, and the $p$ value for the test of close fit was $\mathrm{p}<.001$, providing good model fit. The Comparative Fit index was .79 and the Tucker Lewis index was .77, both indicators of providing poor model fit. The SRMR was .08 suggesting good model fit. Importantly, the nested chi square test comparing this model to the uni-factor model yielded a statistically significant chi-square difference, $\chi^{2} \operatorname{diff}(10)=2791.66, p<.001$, a result that suggests the five-factor model is a better fit. See Table 1 for a summary for the No Exposure CFA. 
Table 1. Comparison of Alternative Confirmatory Factor Analysis Models for Perceptions of Organizational Personality

\begin{tabular}{|c|c|c|c|c|c|c|c|c|}
\hline Model & $\chi^{2}(d f)$ & $\Delta \chi^{2}(d f)$ & AIC & RMSEA & $p$ Close & CFI & TLI & SRMR \\
\hline \multicolumn{9}{|c|}{ Website Condition } \\
\hline Uni-factor & $\chi^{2}(496)=$ & --- & 5253.45 & .11 & $<.001$ & .79 & .76 & .42 \\
\hline Five-factor & $\begin{array}{l}5123.45 \\
\chi^{2}(486)= \\
4128.98\end{array}$ & $\begin{array}{l}\chi^{2}(10)= \\
994.46\end{array}$ & 4278.98 & .10 & $<.001$ & .83 & .82 & .07 \\
\hline \multicolumn{9}{|c|}{ Facebook Condition } \\
\hline Uni-factor & $\begin{array}{l}\chi^{2}(496)= \\
4578.57\end{array}$ & --- & 4708.57 & .11 & $<.001$ & .75 & .73 & .33 \\
\hline Five-factor & $\begin{array}{l}\chi^{2}(486)= \\
3288.13\end{array}$ & $\begin{array}{l}\chi^{2}(10)= \\
1290.45\end{array}$ & 3438.13 & .09 & $<.001$ & .83 & .81 & .07 \\
\hline \multicolumn{9}{|c|}{ No Exposure Condition } \\
\hline Uni-factor & $\begin{array}{l}\chi^{2}(496)= \\
4707.41\end{array}$ & --- & 4933.21 & .17 & $<.001$ & .38 & .34 & .14 \\
\hline Five-factor & $\begin{array}{l}\chi^{2}(486)= \\
1915.75\end{array}$ & $\begin{array}{c}\chi^{2}(10)= \\
2791.66\end{array}$ & 2065.75 & .09 & $<.001$ & .79 & .77 & .08 \\
\hline
\end{tabular}

Note. All chi-square values are significant a $p<.001 . \Delta \chi^{2}=$ chi-square goodness-of-fit difference between uni-factor and five-factor model. AIC $=$ Akaike information criterion. RMSEA $=$ root-mean-square error of approximation. $p$ close $=p$ of Close Fit. CFI $=$ comparative fit index. TLI $=$ Tucker-Lewis index. SRMR $=$ standardized root mean square residual.

\section{Aggregating Individual Responses}

Before proceeding with aggregate analysis, data collected during phase one was cleaned. This included removing lines of data that reflected insufficient responses to the test items, reverse-coding the appropriate items, and scoring the scales of each of the variables included in the hypotheses, and assessing the internal reliability of the computed scales.

The data collected in phase one was used to create averaged scores of perceptions of OP for each organization. This was done in order to eliminate idiosyncrasies across participants in an effort to generate the general public's perception of OP for each company. Although averaging the ratings across students largely eliminates the influence 
of individual idiosyncratic views, the interclass correlation coefficient (ICC) across raters as well as the mean and standard deviation for each company were examined to identify possible outliers. ICC was computed using SPSS to assess agreement across raters for each company. Most of the ICC values were above a satisfactory level (ICC > .70) with only a few cases slightly below the benchmark (ICC > .60) across the three conditions. For the Website condition, $81 \%$ of the ICC values were above .7 and $19 \%$ were above .6 . For the Facebook condition, $84 \%$ of the ICC values were above .7, 13\% were above .6, and $3 \%$ were above .5 . Lastly, $78 \%$ of the ICC values were above .7 for the No Exposure condition and the remaining $22 \%$ were above .6. Closer examinations of the lower ICC values suggest true differences in perceptions of $\mathrm{OP}$ - and not rater or calculation errors. See Appendix K for a summary of the ICC values for each company.

The averaged perceptions of OP scores are used for all subsequent hypotheses testing ( $\mathrm{n}=102$ for Website and Facebook conditions; $\mathrm{n}=50$ for No Exposure condition). Table, 
Table, and Table 4 show the summary descriptive statistics of the aggregated agreement OP scores for the Website, Facebook, and No Exposure conditions, respectively. 
Table 2. Means, Standard Deviations, and Correlations of Perceptions of Organizational Personality for the Website Condition

\begin{tabular}{lrrrrrrrr}
\hline & Mean & $S D$ & P & BS & I & D & T & S \\
\hline Prestige & 4.53 & .47 & .83 & -- & -- & -- & -- & -- \\
Boy Scout & 3.80 & .29 & $.55^{* *}$ & .94 & -- & -- & -- & -- \\
Innovation & 3.44 & .38 & $.64^{* *}$ & $.50^{* *}$ & .90 & -- & -- & -- \\
Dominance & 3.65 & .34 & $.68^{* *}$ & $.60^{* *}$ & $.54^{* *}$ & .86 & -- & -- \\
Thrift & 2.24 & .33 & $-.45^{* *}$ & $-.41^{* *}$ & $-.63^{* *}$ & $-.36^{* *}$ & .92 & -- \\
Style & 2.94 & .51 & $.45^{* *}$ & $.33^{* *}$ & $.64^{* *}$ & $.45^{* *}$ & $-.40^{* *}$ & .96 \\
\hline
\end{tabular}

Note. Internal reliability estimates provided along the diagonal. Scores range from $1=$ strongly disagree to $5=$ strongly agree.

$* p<.05 . * * p<.01$.

Table 3. Means, Standard Deviations, and Correlations of Perceptions of Organizational Personality for the Facebook Condition $(\mathrm{N}=102)$

$\begin{array}{llllllll}\text { Mean } & S D & \mathrm{P} & \mathrm{BS} & \mathrm{I} & \mathrm{D} & \mathrm{T} & \mathrm{S}\end{array}$

\begin{tabular}{lrrrrrrrr}
\hline Prestige & 4.40 & 1.15 & .83 & -- & -- & -- & -- & -- \\
Boy Scout & 3.90 & .32 & $.46^{* *}$ & .91 & -- & -- & -- & -- \\
Innovation & 3.57 & .39 & $.58^{* *}$ & $.59^{* *}$ & .89 & -- & -- & -- \\
Dominance & 3.67 & .37 & $.68^{* *}$ & $.52^{* *}$ & $.67^{* *}$ & .81 & -- & -- \\
Thrift & 2.25 & .35 & $-.63^{* *}$ & $-.42^{* *}$ & $-.55^{* *}$ & $-.56^{* *}$ & .91 & -- \\
Style & 3.13 & .58 & $.43^{* *}$ & $.26^{* *}$ & $.67^{* *}$ & $.54^{* *}$ & $-.47^{* *}$ & .95
\end{tabular}

Note. Internal reliability estimates provided along the diagonal. Scores range from $1=$ strongly disagree to $5=$ strongly agree.

$* p<.05 . * * p<.01$. 
Table 4. Means, Standard Deviations, and Correlations of Perceptions of Organizational Personality for the No Exposure Condition $(\mathrm{N}=50)$

\begin{tabular}{lcccccccc}
\hline & Mean & SD & P & BS & I & D & T & S \\
\hline Prestige & 4.30 & .68 & .79 & -- & -- & -- & -- & -- \\
Boy Scout & 3.68 & .28 & $.50^{* *}$ & .89 & -- & -- & -- & -- \\
Innovation & 3.37 & .41 & $.49^{* *}$ & $.49^{* *}$ & .84 & -- & -- & -- \\
Dominance & 3.80 & .28 & $.43^{* *}$ & $.51^{* *}$ & $.66^{* *}$ & .78 & -- & -- \\
Thrift & 2.40 & .44 & -.27 & $-.36^{* *}$ & $-.43^{* *}$ & $-.52^{* *}$ & .91 & -- \\
Style & 3.09 & .63 & $.43^{* *}$ & $.50^{* *}$ & $.76^{* *}$ & $.55^{* *}$ & $-.32^{*}$ & .94 \\
\hline
\end{tabular}

Note. Internal reliability estimates provided along the diagonal. Scores range from $1=$ strongly disagree to $5=$ strongly agree.

$* p<.05 . * * p<.01$.

\section{Research Questions 1-5: Development of Objective Indicators for OP}

This section covers the results obtained in phase two which was focused on developing objective ratings of organizational personality for each company through the use of objective indicators. Results are discussed in order that they were performed (Step 1 through Step 3).

\section{Step One: Indicator Ratings}

For step one, the principal investigator scored the indicators summarized in research questions 1-5 using the website and Facebook screenshots. This was completed for all of the companies in the database $(\mathrm{N}=102)$. Any questionable ratings were directed to a subject matter expert for further review. Due to low occurrences in website screenshots $(\mathrm{n} \leq 5)$, the following indicators were omitted from subsequent analyses: spelling mistakes, audio/music on webpage, information about bonus-system listed, and total visitor count. Similarly, the following Facebook indicators were omitted from subsequent analyses: spelling mistakes, posts containing audio/music, information about 
bonus-system posted, and option of viewing profile in a different language. At the conclusion of this step, each company had objective scores for each of the indicators listed in RQ1-5.

\section{Step Two: Content Validation}

A group of 14 Subject Matter Experts (SMEs) were chosen to participate in the sort task. SME ratings were then compiled to reflect the total number of raters that selected each OP dimension for the target indicator. A minimum of five SMEs (or 71\%) needed to select a dimension in order for the indicator to be retained as representing the dimension specified in RQ1-5. Additionally, if 71\% agreement was achieved, an indicator was classified as representing an OP dimension, even if the item was not previously classified to that dimension based on RQ1-5. It is possible that indicators reflect more than one dimension. This was done for both the Website indicators and the Facebook profile indicators. The results obtained from the content validation task were used to revise the original classification (i.e., which OP dimension(s) the indicator represents) of the Website and Facebook indicators. 
Table 1 and Table 2, present summaries of the Website and Facebook sort task, respectively. For a detailed breakdown the sort task results of each indicator, refer to Appendix I for Website indicators and Appendix J for Facebook indicators. 
Table 1. Summary of Sort Task Results for Website Indicators

\begin{tabular}{lccrrr}
\hline \multicolumn{5}{c}{ Organizational Personality Dimension } \\
\hline & BS & I & D & T & \multicolumn{1}{c}{ S } \\
\hline $\begin{array}{l}\text { Original } \\
\text { classification }\end{array}$ & 14 & 14 & 13 & 8 & 11 \\
$\begin{array}{l}\text { Sort Task } \\
\text { Classification }\end{array}$ & 10 & 6 & 8 & 4 & 7 \\
$\quad$ Original Retained & 10 & 6 & 7 & 4 & 6 \\
$\quad$ Original Deleted & 4 & 8 & 5 & & 5 \\
$\quad$ New Added & 0 & 0 & 1 & 0 & 1 \\
Item Retention Rate & $71.4 \%$ & $42.9 \%$ & $53.9 \%$ & $50.0 \%$ & $54.6 \%$ \\
\hline
\end{tabular}

Note. Sort task classification based on 5 out of 7 agreement (71\%).

Table 2. Summary of Sort Task Results for Facebook Indicators

\begin{tabular}{lrrrrrr}
\hline \multicolumn{7}{c}{ Organizational Personality Dimension } \\
\hline & \multicolumn{1}{c}{ BS } & I & D & T & S \\
\hline Original classification & 15 & 14 & 14 & 9 & 11 \\
Sort Task Classification & 14 & 11 & 9 & 6 & 9 \\
$\quad$ Original Retained & 11 & 8 & 8 & 5 & 6 \\
Original Deleted & 4 & 6 & 6 & 4 & 5 \\
$\quad$ New Added & 3 & 3 & 1 & 1 & 3 \\
Item Retention Rate & $73.3 \%$ & $57.1 \%$ & $57.1 \%$ & $55.6 \%$ & $54.5 \%$ \\
\hline
\end{tabular}

Note. Sort task classification based on 5 out of 7 agreement (71\%).

This revised version of the sort task indicator classification was used for the empirical validation in the following step.

\section{Step Three: Empirical Validation}

The final step entailed an empirical validation of the objective OP indicator classification, as supported by the content validation, through structural equation 
modeling (SEM). Preliminary analyses were performed using SPSS prior to creating the confirmatory factor models in AMOS. All continuous variables $(\mathrm{N}=36)$ were examined to assess the normality of each variable as well as to identify possible outliers.

Specifically, normality was assessed by obtaining skewness and kurtosis values, comparing trimmed versus original means, performing a test of normality using the Kolmogorov-Smirnov statistic, as well as examining histograms, and boxplots. After examining the data, and due to the nature of the variables (i.e., skewed counts of various elements), square-root transformations were performed on all of the continuous variables. Additionally, all indicators were standardized by converting the square root transformed scores into z-scores in order to convert all of the items to a common scale. Despite these transformations, however, the following Website indicators were removed from the CFA analysis due to non-normal distributions: presence of athlete/celebrity endorsement, frequency of the word 'winning,' frequency of the word 'risk,' and the presence of a discussion forum. Of the excluded Website variables, none were present in more than five corporate webpages. Similarly, the following Facebook indicators were removed from the CFA analysis: presence of contact information, number of posts with athlete/celebrity endorsements, number of people 'talking about' the page, number of page 'likes,' length of 'about us' section, and frequency of the word 'budget.' Of the excluded Facebook variables, lack of normality was mostly due to low occurrences within the company profiles, as well as high counts for those with large values.

To empirically test the factor structure factor structure of organizational personality for Website and Facebook indicators, two separate Confirmatory Factor Analyses (CFA) were run using AMOS. This analysis determines how well the proposed 
factor structure of the indicators fit the data. In order to establish that a five-factor model is supported, two models were run and compared to one another to determine which has the better model fit. The first model was a uni-factor model (i.e., all indicators for every OP dimension loading on a latent variable) and the second model was a five-factor model (i.e., all indicators loading on each respective dimension as classified by the content validation). Although several indices were used, the comparative fit index (CFI) and the root mean square error of approximation (RMSEA) were our primary focus for model evaluation in the current study as recommended by researchers (Thompson, 2000). Because models that differ in regard to the number of latent factors were not necessarily nested, we utilized the Akaike information criterion (AIC) index versus a $\chi^{2}$ difference test to compare our CFA models (Brown, 2006). Additionally, if good model fit was achieved for the five-factor structure, a more detailed examination of each OP dimension was performed by reviewing factor loadings for each indicator. Indicators with low factor loadings were further examined for possible deletion.

\section{Website Indicator CFA.}

Results of the CFA analyses indicate that the single factor model in which all items loaded on a common latent construct did not fit the data well: $\chi^{2}(90)=84.66, p<$ $.001 ; \mathrm{CFI}=.32$; TLI $=.58$; $\mathrm{RMSEA}=.12(90 \% \mathrm{CI}$ of RMSEA $=.10-.14)$. The fivefactor model was then tested in which the five latent constructs were allowed to freely

covary. This model fit the data well: $\chi^{2}(80)=84.66, \mathrm{p}=.34 ; \mathrm{CFI}=.95 ; \mathrm{RMSEA}=.02$ $(90 \%$ CI of RMSEA $=.00-.06)$. The AIC displayed the following results: 1 -factor model $=272.51 ; 5$-factor model $=164.66$. Collectively these results suggest that the five-factor model fit the data well and possessed the stronger theoretical rationale. Standardized 
regression weights for this model are presented in Figure 1. See Table 3 for summary for the Website CFA results.

Facebook Indicator CFA. Results of the CFA analyses indicate that the single factor model in which all items loaded on a common latent construct did not fit the data well: $\chi^{2}(140)=302.12, \mathrm{p}<.001 ; \mathrm{CFI}=.31 ; \mathrm{TLI}=.22 ; \mathrm{RMSEA}=.10(90 \% \mathrm{CI}$ of RMSEA $=.08-.12$ ). The five-factor model was then tested in which the latent constructs were allowed to freely covary. This model fit the data marginally good: $\chi^{2}(140)=$ 174.76, $\mathrm{p}<.05 ; \mathrm{CFI}=.84 ; \mathrm{TLI}=.81 ; \mathrm{RMSEA}=.05(90 \% \mathrm{CI}$ of $\mathrm{RMSEA}=.02-.07)$. The AIC displayed the following results: 1 -factor model $=382.12 ; 5$-factor model $=$ 274.76. Collectively these results suggest that the five-factor model fit the data better than the single-factor model, and possessed the stronger theoretical rationale. Standardized regression weights for this model are presented in Figure 2. See Table 3 for summary for the Facebook CFA results.

Table 3. Comparison of Alternative Confirmatory Factor Analysis Models for Objective Indicators of Organizational Personality

\begin{tabular}{|c|c|c|c|c|c|c|}
\hline Model & AIC & RMSEA & $p$ Close & CFI & TLI & SRMR \\
\hline \multicolumn{7}{|c|}{ Website Indicators } \\
\hline Uni-factor & 272.51 & .12 & .00 & .32 & .58 & .19 \\
\hline Five-factor & 164.66 & .02 & .84 & .95 & .93 & .07 \\
\hline \multicolumn{7}{|c|}{ Facebook Indicators } \\
\hline Uni-factor & 382.12 & .10 & .00 & .31 & .22 & .17 \\
\hline Five-factor & 274.76 & .05 & .50 & .84 & .81 & .08 \\
\hline
\end{tabular}

Note. $\mathrm{AIC}=$ Akaike information criterion. RMSEA $=$ root-mean-square error of approximation. $p$ close $=p$ of Close Fit. $\mathrm{CFI}=$ comparative fit index. TLI $=$ Tucker-Lewis index. SRMR = standardized root mean square residual. 
Figure 1. Website Indicator CFA Model and Associated Standardized Regression Weights

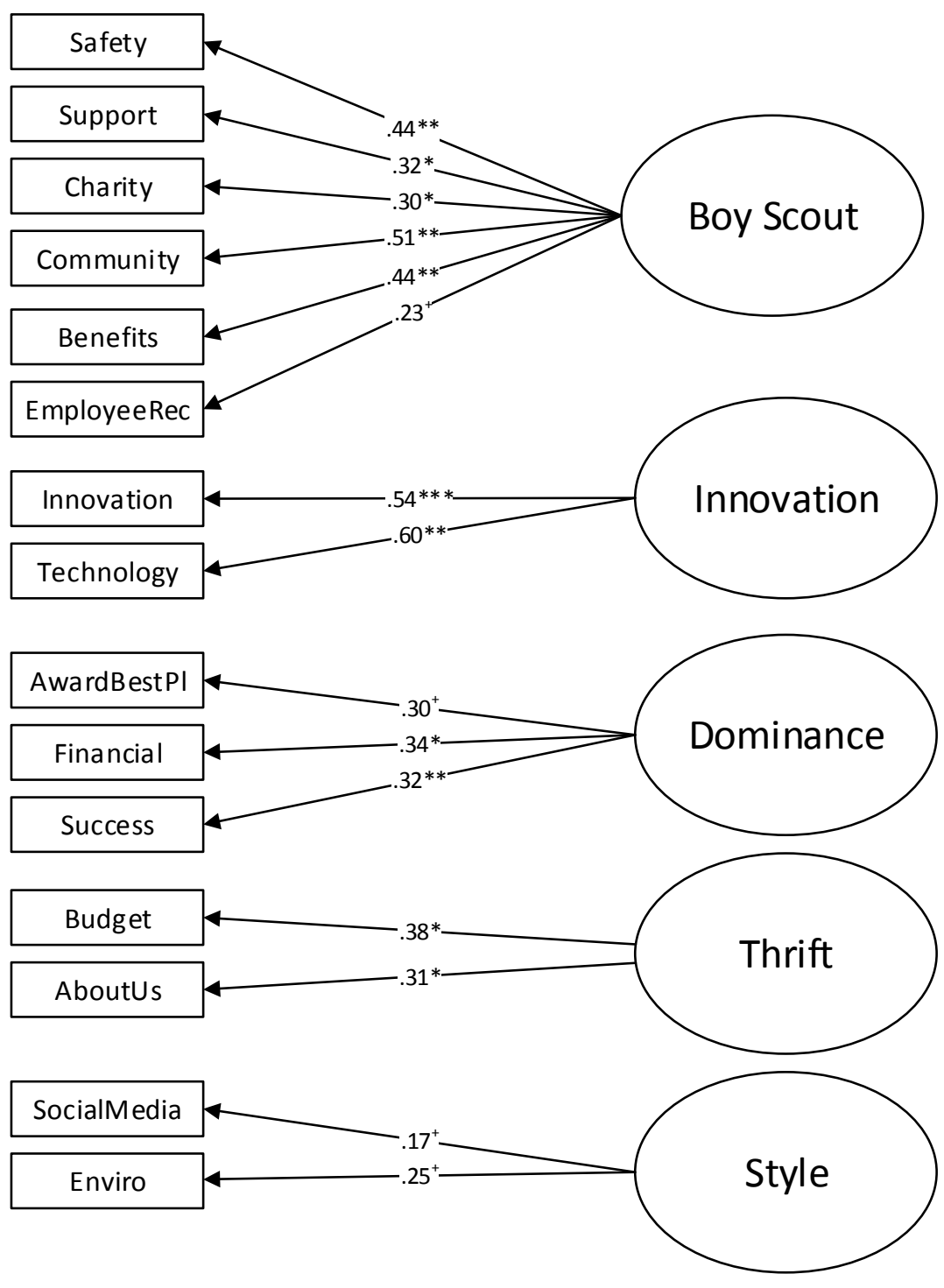

$* p<.05 . * * p<.01$. 
Figure 2. Facebook Indicator CFA Model and Associated Standardized Regression

\section{Weights}

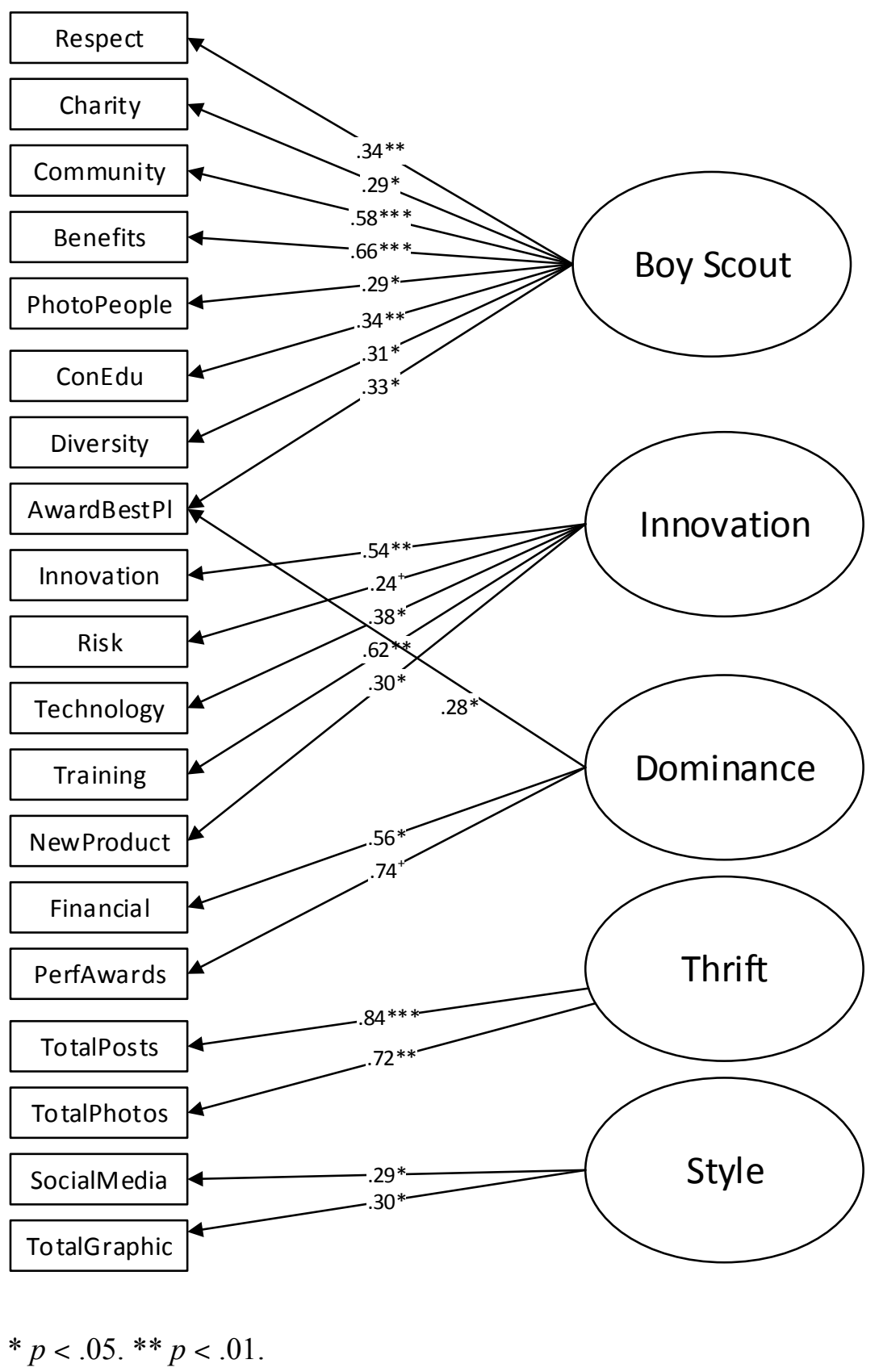

Following the CFAs, a composite score was determined by averaging for each OP dimension comprised of all of the indicators in the 5-factor model. This refined score, as modified by both the content validation and the empirical validation, serves as the 
objective OP score for each organization. At the conclusion of phase two, each company had the following scores associated with it: interrater-averaged perceptions of OP scores for the three experimental conditions, individual indicator ratings for Website and Facebook, and composite objective OP ratings for Websites and Facebook profiles.

\section{Hypothesis 1 and 2: Testing Convergence between Self-Report and Objective Scores}

External validation of the Website and Facebook indicators was performed in a method consistent with the multitrait-multimethod matrix (MTMM) (Campbell \& Fiske, 1959). This approach is designed to assess convergent validity, divergent validity, and method variance when at least two traits are assessed with at least two methods. Due to sample size limitations, as well as hypothesized differences, the analyses focused on convergent validation of the Website and Facebook indicators separately. Convergent validation posits that measures of the same trait should converge, or agree. To do this, correlations of the same-trait (e.g., each organizational personality dimension) with different measurement methods (e.g., self-reported perceptions and objective indicators) were examined.

\section{Hypothesis 1: Website Condition Convergence}

Hypothesis one postulates that perceptions of image based on exposure to corporate websites will converge with objective scores of image. Pearson correlation analysis was performed to assess convergence between perceptions of OP and objective scores of OP for the Website condition. For the Thrift dimension, results indicated a significant positive relationship between perceptions of Thrift and objective Thrift scores, $r=.20, p<.05$. The small effect size, however, only shows moderate support for convergent validity. Furthermore, none of the other dimensions were statistically 
significant, thus suggesting no convergent validity for perception and objective OP scores. Interestingly, the Boy Scout objective scores were significantly negatively related to perceptions of Innovation and Style as well as positively related to perceptions of Thrift. Results do not provide support for this hypothesis. See Table 4 for a summary of the Website convergence analysis.

Table 4. Summary of Convergent Analysis for the Website Condition ( $\mathrm{N}=102)$ Perceptions of OP Scores

\begin{tabular}{lrrrrr}
\hline Objective OP & \multicolumn{1}{c}{ BS } & \multicolumn{1}{l}{$\mathrm{I}$} & \multicolumn{1}{l}{$\mathrm{T}$} & \multicolumn{2}{l}{$\mathrm{D}$} \\
\hline Boy Scout & -.01 & $-.26^{* *}$ & -.17 & $.28^{* *}$ & $-.37^{* * *}$ \\
Innovation & -.11 & $\underline{.11}$ & .07 & -.05 & -.05 \\
Dominance & .11 & -.05 & .12 & -.12 & -.05 \\
Thrift & .12 & -.09 & .05 & $\underline{.20^{*}}$ & .12 \\
Style & .07 & .13 & -.02 & .01 & -.11 \\
\hline
\end{tabular}

Note. Underlined values represent coefficients for matching OP dimensions.

$* p<.05 . * * p<.01 * * * p<.001$.

\section{Supplementary Analysis for Hypothesis 1}

In addition to examining convergence with the composite objective OP scores, convergence between individual objective indicators and perceptions of $\mathrm{OP}$ was also performed in order to examine the relationships at the individual indicator level in addition to the composite indicator level. This analysis was added in response to the marginally good results obtained from the objective indicator CFA analysis. Only the indicators that were included in the final objective Website OP score composites were examined. Results parallel the findings obtained with the composite scores. See Table 5 for a summary of the Pearson correlation analyses at the individual indicator level. 
Table 5. Summary of Supplementary Convergent Analysis for the Website Condition (N $=102)$

\begin{tabular}{|c|c|c|c|c|c|}
\hline \multirow{2}{*}{ Website Objective Indicators } & \multicolumn{5}{|c|}{ Perceptions of OP Scores } \\
\hline & $\mathrm{BS}$ & $\mathrm{I}$ & $\mathrm{D}$ & $\mathrm{T}$ & $\mathrm{S}$ \\
\hline \multicolumn{6}{|l|}{ Boy Scout } \\
\hline Frequency of word 'safety' & -.07 & -.12 & -.03 & $.21 *$ & $-.26 * *$ \\
\hline Frequency of word 'support' & .02 & $-.27 * *$ & -.14 & $.27 * *$ & $-.34 * *$ \\
\hline Information about charity work posted & .00 & -.06 & -.10 & .05 & -.08 \\
\hline Information about community involvement posted & -.09 & $-.20 *$ & -.13 & $.27 * *$ & $-.26^{* *}$ \\
\hline Information about employee benefits posted & -.05 & -.12 & $-.17^{+}$ & .10 & $-.19^{+}$ \\
\hline Presence of an employee recognition program & .05 & -.06 & .03 & -.03 & -.03 \\
\hline \multicolumn{6}{|l|}{ Innovation } \\
\hline Frequency of word 'innovation' & .00 & .10 & .11 & -.10 & .00 \\
\hline Frequency of word 'technology' & $-.18^{+}$ & .08 & .00 & .01 & -.09 \\
\hline \multicolumn{6}{|l|}{ Dominance } \\
\hline Awards for best places to work listed & .13 & -.03 & .05 & -.05 & -.10 \\
\hline Financial information listed & .00 & -.08 & .13 & -.05 & .05 \\
\hline Frequency of word 'success' & .07 & .02 & .02 & -.10 & -.03 \\
\hline \multicolumn{6}{|l|}{ Thrift } \\
\hline Frequency of word 'budget' & $.18^{+}$ & -.13 & -.04 & .16 & .09 \\
\hline Length of 'about us' section (reversed) & -.02 & -.00 & .11 & .12 & .09 \\
\hline \multicolumn{6}{|l|}{ Style } \\
\hline Number of links to other [social] media sites & $.17^{+}$ & .15 & .05 & -.08 & -.09 \\
\hline Information about environmental-awareness listed & -.08 & .03 & -.08 & .09 & -.06 \\
\hline
\end{tabular}

Note. Underlined values represent coefficients for matching OP dimensions.

${ }^{+} p<.10 * p<.05 . * * p<.01 * * * p<.001$.

\section{Hypothesis 2: Facebook Condition Convergence}

Hypothesis two posits that perceptions of image based on exposure to company

Facebook profiles will converge with objective scores of image. Pearson correlation analysis was performed to assess convergence between perceptions of OP and objective scores of OP for the Facebook condition. For the Dominance, $r=.37, p<.001$, and Thrift, $r=.29, p<.01$, dimensions, results indicated a significant positive relationship 
between perceptions of OP and objective scores, thus providing evidence for convergence. Additionally, marginally significant positive relationships were found for the three remaining OP dimensions, Boy Scout, $r=.17, p<.10$, Innovation, $r=.17, p<$ .10 , and Style, $r=.17, p<.10$. Except for the Dominance dimension, the objective scores did not display stronger, positive relationships with other perceptions of OP dimensions. In addition, the objective score did not show positive significant relationships with any other dimension perception of OP dimension. Results provide partial support for this hypothesis, particularly for the Dominance and Thrift dimensions. See Table 6 for a summary of the Website convergence analysis.

Table 6. Summary of Convergent Analysis for the Facebook Condition $(\mathrm{N}=102)$

\begin{tabular}{lrrrrr}
\hline \multicolumn{5}{c}{ Perceptions of OP Scores } \\
\hline Objective OP & \multicolumn{1}{c}{$\mathrm{BS}$} & $\mathrm{I}$ & $\mathrm{D}$ & \multicolumn{1}{l}{$\mathrm{T}$} & \multicolumn{1}{c}{$\mathrm{S}$} \\
\hline Boy Scout & \multicolumn{1}{c}{$.17^{+}$} & .12 & $.21^{*}$ & $-.18^{+}$ & .14 \\
Innovation & .08 & $.17^{+}$ & .06 & -.06 & -.01 \\
Dominance & $.31^{* *}$ & $.29^{* *}$ & $.37^{* * *}$ & $-.23^{*}$ & .1 \\
Thrift & -.15 & $-.30^{* *}$ & $-.48^{* * *}$ & $\underline{.29 * *}$ & $-.40^{* * *}$ \\
Style & .07 & .15 & .1 & -.08 & $.17^{+}$ \\
\hline
\end{tabular}

Note. Underlined values represent coefficients for matching OP dimensions.

${ }^{+} p<.10 * p<.05 * * p<.01 * * * p<.001$

\section{Supplementary Analysis for Hypothesis 2}

In addition to examining convergence with the composite objective OP scores, convergence between individual objective indicators and perceptions of $\mathrm{OP}$ was also performed. This analysis was added in response to the marginally good results obtained from the objective indicator CFA analysis. Only the indicators that were included in the final objective Facebook OP score composites were examined. See Table 7 for a summary of the Pearson correlation analyses at the individual indicator level. As 
suggested by the marginally good fit of the CFA, results highlight differences among objective indicators grouped to the same dimension. For example, despite the content and empirical validation of the objective scores, only select indicators relate to the perception of OP score for each dimension.

Table 7. Summary of Supplementary Convergent Analysis for the Facebook Condition $(\mathrm{N}=102)$

\begin{tabular}{|c|c|c|c|c|c|}
\hline \multirow[b]{2}{*}{ Facebook Objective Indicators } & \multicolumn{5}{|c|}{ Perceptions of OP Scores } \\
\hline & $\mathrm{BS}$ & $\mathrm{I}$ & $\mathrm{D}$ & $\mathrm{T}$ & $\mathrm{S}$ \\
\hline \multicolumn{6}{|l|}{ Boy Scout } \\
\hline Frequency of word 'respect' & .07 & .14 & .12 & $-.19^{+}$ & $.22 *$ \\
\hline Information about charity work posted & $-\underline{.14}$ & -.10 & -.14 & .03 & -.05 \\
\hline Information about community involvement posted & .06 & -.09 & .07 & .04 & -.02 \\
\hline Information about employee benefits posted & .04 & .07 & .12 & -.02 & .11 \\
\hline Number of photos posted of people & $.31 * *$ & $.23 *$ & $.27 * *$ & $-.26 * *$ & .09 \\
\hline Information about continuing education posted & -.03 & .02 & .00 & .05 & .04 \\
\hline Awards for best places to work posted & .16 & .09 & $.18^{+}$ & -.08 & -.03 \\
\hline Frequency of word 'diversity' & $.24^{*}$ & .14 & $.23 *$ & $-.31 * *$ & $.21 *$ \\
\hline \multicolumn{6}{|l|}{ Innovation } \\
\hline Frequency of word 'innovation' & -.11 & $\underline{-.05}$ & -.17 & .15 & -.18 \\
\hline Frequency of word 'risk' & .13 & $.22 *$ & .05 & .03 & -.02 \\
\hline Frequency of word 'technology' & -.08 & .00 & .02 & .03 & -.04 \\
\hline Information about training/education opportunities posted & .11 & -.12 & -.10 & .01 & $-.25^{*}$ \\
\hline Posts of advertisements for new product/service launches & .15 & $.38^{* *}$ & $.34 * *$ & $-.35 * *$ & $.46^{* *}$ \\
\hline \multicolumn{6}{|l|}{ Dominance } \\
\hline Awards for best places to work posted & .16 & .09 & $.18^{+}$ & -.08 & -.03 \\
\hline Postings relating to financial information & .15 & .13 & .15 & $-.20 *$ & -.02 \\
\hline Performance awards listed & $.34 * *$ & $.40 * *$ & $.45^{* *}$ & $-.23 *$ & $.28 * *$ \\
\hline \multicolumn{6}{|l|}{ Thrift } \\
\hline Number of posts by page & $.20 *$ & $.30 * *$ & $.42 * *$ & $-\underline{.27 * *}$ & $.36^{* *}$ \\
\hline Total number of images/ photos on profile & .08 & $.24^{*}$ & $.43 * *$ & $-.25^{*}$ & $.36^{* *}$ \\
\hline \multicolumn{6}{|l|}{ Style } \\
\hline Number of links to other [social] media sites & .09 & .17 & $.20 *$ & -.07 & $.19^{+}$ \\
\hline Number of posts containing graphics/artwork & .01 & .05 & -.05 & -.05 & .07 \\
\hline
\end{tabular}

Note. Underlined values represent coefficients for matching OP dimensions.

${ }^{+} p<.10 * p<.05 * * p<.01 * * * p<.001$ 


\section{Hypothesis 3 and 4: Source Differentiation Analyses across Recruitment Media}

Source differentiation analyses were performed to establish differences in levels of organizational personality dimensions across different recruitment sources. For perceptions of OP, a repeated-measures multivariate analysis of variance (R-MANOVA) was performed to assess differences in perceptions of OP across the treatment conditions. If the MANOVA showed significant differences, follow-up one way repeated-measures analyses of variance (ANOVA) were conducted for each dimension separately. For objective OP scores, Pearson correlation coefficients were examined to asses differences in objective Website OP scores and objective Facebook OP scores.

\section{Hypothesis 3: Perceptions of OP Source Differentiation}

Hypothesis three suggests that perceptions of OP scores will differ depending on which (if any) recruitment material participants were exposed to (i.e. Website condition, Facebook condition, No Exposure condition). A repeated-measures multivariate analysis of variance (R-MANOVA) was conducted to determine the effect of three types of recruitment methods on perceptions of organizational personality across five dimensions (Boy Scout, Innovation, Dominance, Thrift, and Style). Due to the smaller sample size in the No Exposure group, the sample size for this analysis was $n=50$ companies.

Significant differences were found on the dependent measures, Wilks's $\Lambda=.37, F(10$, $40)=6.85, p<.001$. The multivariate $\eta^{2}$ based on Wilks's $\Lambda$ was strong, .63. Table 8 contains the means and the standard deviations of the organizational personality dimensions for the three recruitment methods. 
Table 8. Means and Standard Deviations of OP dimensions across the Three Recruitment Methods $(\mathrm{N}=50)$

\begin{tabular}{lllllll}
\hline & Website & \multicolumn{3}{c}{ Facebook } & \multicolumn{2}{c}{ No Exposure } \\
\hline OP Dimension & $M$ & $S D$ & $M$ & $S D$ & $M$ & $S D$ \\
\hline Boy Scout & $3.93^{\mathrm{b}}$ & 0.26 & $3.93^{\mathrm{b}}$ & 0.29 & $3.68^{\mathrm{a}}$ & 0.28 \\
Innovation & $3.54^{\mathrm{b}}$ & 0.38 & $3.68^{\mathrm{b}}$ & 0.36 & $3.37^{\mathrm{a}}$ & 0.41 \\
Dominance & 3.82 & 0.31 & 3.82 & 0.28 & 3.80 & 0.28 \\
Thrift & $2.19^{\mathrm{b}}$ & 0.35 & $2.17^{\mathrm{b}}$ & 0.35 & $2.40^{\mathrm{a}}$ & 0.44 \\
Style & $3.15^{\mathrm{a}}$ & 0.55 & $3.39^{\mathrm{b}}$ & 0.63 & $3.09^{\mathrm{a}}$ & 0.63 \\
\hline
\end{tabular}

Note. Values with different superscript letters denote significant differences using Bonferroni procedure, $\mathrm{p}<.05$.

Given the significant results of the MANOVA, repeated-measures univariate analyses of variances (ANOVA) on each OP dimension were conducted as follow-up. Additionally, for significant ANOVAs, Bonferroni's pairwise comparison of means determine which recruitment methods differed. Accordingly, each pairwise comparison was tested at $p<.017$.

The ANOVA on perceptions of Boy Scout indicated a significant recruitment method effect, Wilks's $\Lambda=.61, F(2,48)=15.29, p<.001$, multivariate $\eta^{2}=.39$. Pairwise comparisons suggest that perceptions of Boy Scout were significantly lower for companies rated in the No Exposure condition $(M=3.68)$ than in the Website $(M=3.93)$ and Facebook conditions $(M=3.93)$. Results provide partial support for $\mathrm{H} 3$ specific to the Boy Scout OP dimension.

The ANOVA on perceptions of Innovation indicated a significant recruitment method effect, Wilks's $\Lambda=.65, F(2,48)=13.07, p<.001$, multivariate $\eta^{2}=.35$. Pairwise comparisons suggest that perceptions of Innovation were significantly lower for companies rated in the No Exposure condition $(M=3.37)$ compared to those rated in the 
Website $(M=3.54)$ and Facebook conditions $(M=3.68)$. Consequently, results provide partial support for $\mathrm{H} 3$ specific to the Innovation OP dimension.

The ANOVA on perceptions of Dominance indicated no significant recruitment method effect, Wilks's $\Lambda=.99, F(2,48)=.16, p=.85$, multivariate $\eta^{2}=.01$. Therefore, results do not provide support for $\mathrm{H} 3$ specific to the Dominance OP dimension.

The ANOVA on perceptions of Thrift indicated a significant recruitment method effect, Wilks's $\Lambda=.73, F(2,48)=8.77, p<.01$, multivariate $\eta^{2}=.27$. Pairwise comparisons suggest that perceptions of Thrift were significantly higher for companies rated in the No Exposure condition $(M=2.40)$ than the Website $(M=2.19)$ and Facebook conditions $(M=2.17)$. Results provide partial support for $\mathrm{H} 3$ specific to the Thrift OP dimension.

Lastly, the ANOVA on perceptions of Style indicated a significant recruitment method effect, Wilks's $\Lambda=.79, F(2,48)=6.35, p<.01$, multivariate $\eta^{2}=.21$. Pairwise comparisons suggest that perceptions of Style were significantly higher for companies rated in the Facebook condition $(M=3.39)$ than the Website $(M=3.15)$ and No Exposure conditions $(M=3.09)$. Results provide support for H3 specific to the Style OP dimension.

See Table 8 for a summary of the findings.

\section{Supplementary Analyses for Hypotheses 3}

Since the R-MANOVA to determine the effect of three types of recruitment methods (Website, Facebook, and No Exposure) on perceptions of OP could only be performed on the subset of companies $(\mathrm{N}=50)$ that had data in the No Exposure 
condition, additional analyses were performed to explore differences using the full set of companies $(\mathrm{N}=102)$. The No Exposure condition was omitted to allow for analysis in the full set of organizations. Specifically, a repeated-measures multivariate analysis of variance (R-MANOVA) was performed to determine the effect of two recruitment methods (Website and Facebook) on perceptions of OP for the larger sample of companies $(\mathrm{N}=102)$. Results were consistent with the previous analyses. Significant differences were found on the dependent measures, Wilks's $\Lambda=.83, F(5,97)=4.03, p<$ .01 . The multivariate $\eta^{2}$ based on Wilks's $\Lambda$ was small, .17. Table 9 contains the means and the standard deviations of the organizational personality dimensions for the two recruitment methods.

Table 9. Means and Standard Deviations of OP dimensions across the Two Recruitment Methods $(\mathrm{N}=102)$

\begin{tabular}{lllll}
\hline & Website & \multicolumn{2}{l}{ Facebook } \\
\hline OP Dimension & $M$ & $S D$ & $M$ & $S D$ \\
\hline Boy Scout & 3.80 & .29 & 3.90 & .32 \\
Innovation & $3.44^{\mathrm{a}}$ & .38 & $3.57^{\mathrm{b}}$ & .39 \\
Dominance & 3.65 & .34 & 3.67 & .37 \\
Thrift & 2.24 & .33 & 2.25 & .35 \\
Style & $2.94^{\mathrm{a}}$ & .51 & $3.13^{\mathrm{b}}$ & .58 \\
\hline
\end{tabular}

Note. Values with different superscript letters denote significant differences, $\mathrm{p}<.05$.

Given the significant results, univariate tests on each OP dimension were conducted as follow-up. As with the smaller subset of companies, perceptions of Style were significantly lower for companies rated in the Website condition $(M=2.94, S D=$ $.51)$ compared to those rated in the Facebook condition $(M=3.13)$. Additionally, perceptions of Innovation were significantly lower for companies rated in the Website 
condition $(M=3.44)$ compared to those rated in the Facebook condition $(M=3.57)$.

Given the similarities in the mean differences in the two analyses, it is possible that a lack of statistical power in the smaller sample contributed to the non-significant findings for the Innovation dimension between Website and Facebook. Consistent with previous analyses, there were no significant differences in perceptions of Boy Scout, Dominance, or Thrift for the two recruitment sources. Despite not being able to assess differences with the No Exposure group, results suggest that the analyses from the smaller sample are consistent with findings from the full set of companies, except for the Innovation dimension.

\section{Hypothesis 4: Objective OP Scores Source Differentiation}

Hypothesis four predicted that objective OP scores will differ depending on which recruitment material the objective scores represented (i.e. objective Website scores or objective Facebook scores). Since objective scores were computed using z-score standardized indicators, mean differences could not be examined between Website indicator scores and Facebook indicator scores. Alternatively, differentiation was assessed by examining the linear relationship between the two types of scores. As such, non-significant or negative relationships were expected between matching OP dimensions, thus representing dissimilarity across the two objective measures. Each dimension was assessed separately, resulting in five Pearson correlation analyses.

For the Boy Scout dimension, the Pearson correlation analysis used to test the relationship between objective Website and objective Facebook scores indicated a nonsignificant relationship, $r=-.06, p=.54$. Results suggest that the two objective scores do not have a positive, linear relationship, thus providing support for hypothesis four 
specific to the Boy Scout dimension. For the Innovation dimension, the Pearson correlation analysis indicated a large, positive relationship between the two scores, $r=$ $.49, p<.001$. Results suggest a moderate amount of similarity between the two objective scores, thus rejecting hypothesis four specific to the Innovation dimension. For the Dominance dimension, the Pearson correlation analysis indicated a non-significant relationship, $r=.13, p=.18$. Results suggest that the two objective scores do not have a positive, linear relationship, thus providing support for hypothesis four specific to the Dominance dimension. For the Thrift dimension, the Pearson correlation analysis indicated a medium, positive relationship between the two scores, $r=.32, p<.01$. Results only suggest a small amount of similarity between the two objective scores, thus supporting hypothesis four specific to the Thrift dimension. Lastly, the Pearson correlation analysis indicated a non-significant relationship between the two objective Style scores, $r=-.01, p=.94$, thus providing support for hypothesis four specific to the Style dimension.

\section{Objective Indicator Predictive Validity}

The predictive validity analyses used standard multiple regressions to assess the predictive validity of each indicator on perceptions of organizational personality for each dimension. More specifically, objective indicators used to create the composite objective OP scores (See Appendix L) were regressed on the self-reported perception score of the corresponding OP dimension. This was completed twice, first for the objective Website indicators and then for the objective Facebook indicators. 


\section{Research Question 6: Website Indicator Predictive Validity Regressions}

Research Question six sought to identify the related contribution of the final objective website indicators on each of the OP dimensions. Standard multiple linear regression analyses was used to test the predictive validity of Website objective OP indicators on the respective perception of OP score. The five regression models included: 1) objective Boy Scout indicators predicting perceptions of Boy Scout score; 2) objective Innovation indicators predicting perceptions of Innovation score; 3) objective Dominance indicators predicting perceptions of Dominance score; 4) objective Thrift indicators predicting perceptions of Thrift score; and 5) objective Style indicators predicting perceptions of Style score. Preliminary analyses were conducted to ensure no violation of the assumptions of normality, linearity, and multicolinearity.

None of the regression models were statistically significant: Boy Scout, $F(6,95)$ $=.32, p=.93$; Innovation, $F(2,99)=.61, p=.54$; Dominance, $F(3,98)=.70, p=.56$; Thrift, $F(2,99)=2.10, p=.13$; Style, $F(2,99)=.63, p=.54$. See 
Table 10 through Table 14 for details on each model. Therefore, results suggest that none of the objective website indicators significantly predict perceptions of OP. 
Table 10. Multiple Linear Regression for Objective Website Indicators Predicting Perceptions of Boy Scout $(\mathrm{N}=102)$

\begin{tabular}{|c|c|c|c|c|c|}
\hline Objective Boy Scout Indicators & $B$ & $S E$ & $\beta$ & $t$ & $p$ \\
\hline Frequency of word 'safety' & -.02 & .03 & -.07 & -.65 & .52 \\
\hline Frequency of word 'support' & .02 & .03 & .06 & .50 & .62 \\
\hline Information about charity work posted & .01 & .03 & .03 & .29 & .77 \\
\hline $\begin{array}{l}\text { Information about community } \\
\text { involvement posted }\end{array}$ & -.03 & .03 & -.09 & -.87 & .39 \\
\hline $\begin{array}{l}\text { Information about employee benefits } \\
\text { posted }\end{array}$ & -.01 & .03 & -.04 & -.37 & .71 \\
\hline $\begin{array}{l}\text { Presence of an employee recognition } \\
\text { program }\end{array}$ & .02 & .03 & .06 & .61 & .54 \\
\hline
\end{tabular}

Note. $\mathrm{B}=$ unstandardized coefficient, $\mathrm{SE}=$ standard error, $\beta=$ standardized coefficient.

Table 11. Multiple Linear Regression for Objective Website Indicators Predicting Perceptions of Innovation ( $\mathrm{N}=102)$

\begin{tabular}{|c|c|c|c|c|c|}
\hline Objective Innovation Indicators & B & SE & $\beta$ & $t$ & $p$ \\
\hline Frequency of word 'innovation' & .03 & .04 & .08 & .73 & .47 \\
\hline Frequency of word 'technology' & .02 & .04 & .06 & .56 & .58 \\
\hline
\end{tabular}

Note. $\mathrm{B}=$ unstandardized coefficient, $\mathrm{SE}=$ standard error, $\beta=$ standardized coefficient.

Table 12. Multiple Linear Regression for Objective Website Indicators Predicting Perceptions of Dominance $(\mathrm{N}=102)$

\begin{tabular}{|c|c|c|c|c|c|}
\hline Objective Dominance Indicators & $\mathrm{B}$ & SE & $\beta$ & $t$ & $p$ \\
\hline Awards for best places to work listed & .02 & .03 & .05 & .46 & .65 \\
\hline Financial information listed & .05 & .03 & .14 & 1.34 & .18 \\
\hline Frequency of word 'success' & .01 & .04 & .03 & .33 & .74 \\
\hline
\end{tabular}

Note. $\mathrm{B}=$ unstandardized coefficient, $\mathrm{SE}=$ standard error, $\beta=$ standardized coefficient.

Table 13 Multiple Linear Regression for Objective Website Indicators Predicting Perceptions of Thrift $(\mathrm{N}=102)$

\begin{tabular}{lrrrrr}
\hline Objective Thrift Indicators & B & SE & $\beta$ & $t$ & \multicolumn{1}{l}{$p$} \\
\hline Frequency of word 'budget' & .05 & .03 & .16 & 1.61 & .11 \\
Length of 'about us' section (\# of words) & -.04 & .03 & -.12 & -1.27 & .21
\end{tabular}

Note. $\mathrm{B}=$ unstandardized coefficient, $\mathrm{SE}=$ standard error, $\beta=$ standardized coefficient. 
Table 14. Multiple Linear Regression for Objective Website Indicators Predicting Perceptions of Style $(\mathrm{N}=102)$

\begin{tabular}{llllll}
\hline Objective Style Indicators & $\mathrm{B}$ & $\mathrm{SE}$ & $\beta$ & $t$ & $p$ \\
\hline $\begin{array}{l}\text { Number of links to other [social] media } \\
\text { sites }\end{array}$ & -.05 & .05 & -.09 & -.92 & .36 \\
$\begin{array}{l}\text { Information about environmental- } \\
\quad \text { awareness listed }\end{array}$ & -.03 & .05 & -.06 & -.61 & .54 \\
\hline
\end{tabular}

Note. $\mathrm{B}=$ unstandardized coefficient, $\mathrm{SE}=$ standard error, $\beta=$ standardized coefficient.

\section{Research Question 7: Facebook Indicator Predictive Validity Regressions}

Research Question seven aimed to identify the related contribution of the final objective Facebook indicators on each of the OP dimensions. Standard multiple linear regression analyses were used to test the predictive validity of Facebook objective OP indicators on the respective perception of OP score. The five regression models included: 1) objective Boy Scout indicators predicting perceptions of Boy Scout score; 2) objective Innovation indicators predicting perceptions of Innovation score; 3) objective Dominance indicators predicting perceptions of Dominance score; 4) objective Thrift indicators predicting perceptions of Thrift score; and 5) objective Style indicators predicting perceptions of Style score. Preliminary analyses were conducted to ensure no violation of the assumptions of normality, linearity, and multicolinearity.

For the Boy Scout dimension, the model as a whole explained $15.7 \%$ of the variance in perceptions of Boy Scout, $F(8,93)=2.01, p=.05$. Of the eight objective Facebook indicators, 'number of photos posted of people' was the only significant predictor of perceptions of Boy Scout $(\beta=.26, p<.05)$, while 'frequency of the word diversity' was marginally significant $(\beta=.17, p<.10)$. Results suggest company Facebook profiles with more pictures of people and more instances of the word 'diversity' relate to higher perceptions of Boy Scout. See details in Table 15. 
Table 15. Multiple Linear Regression for Objective Facebook Indicators Predicting Perceptions of Boy Scout $(\mathrm{N}=102)$

\begin{tabular}{|c|c|c|c|c|c|}
\hline Objective Boy Scout Indicators & $B$ & $S E$ & $\beta$ & $t$ & $p$ \\
\hline Frequency of word 'respect' & .02 & .03 & .06 & .64 & .52 \\
\hline Information about charity work posted & -.03 & .03 & -.11 & -1.08 & .28 \\
\hline $\begin{array}{l}\text { Information about community } \\
\text { involvement posted }\end{array}$ & .00 & .03 & .00 & -.04 & .97 \\
\hline $\begin{array}{l}\text { Information about employee benefits } \\
\text { posted }\end{array}$ & .00 & .04 & .00 & .02 & .98 \\
\hline Number of photos posted of people & .09 & .04 & .26 & 2.6 & .01 \\
\hline $\begin{array}{l}\text { Information about continuing education } \\
\text { posted }\end{array}$ & -.01 & .03 & -.03 & -.24 & .81 \\
\hline Awards for best places to work posted & .01 & .03 & .03 & .24 & .81 \\
\hline Frequency of word 'diversity' & .06 & .03 & .17 & 1.67 & .09 \\
\hline
\end{tabular}

Note. $\mathrm{B}=$ unstandardized coefficient, $\mathrm{SE}=$ standard error, $\beta=$ standardized coefficient.

For the Innovation dimension, the model as a whole explained $22.1 \%$ of the variance in perceptions of Innovation, $F(5,96)=5.44, p<.001$. Of the five objective Facebook indicators, 'frequency of the word risk' $(\beta=.27, p<.01)$ and 'posts of advertisements for new products or service launches' $(\beta=.40, p<.001)$ were significant predictors of perceptions of Innovation. Results suggest that more instances of the word 'risk' in company Facebook posts and the existence of posts advertising new products or services relate to higher perceptions of Innovation. See details in

Table 16. Multiple Linear Regression for Objective Facebook Indicators Predicting Perceptions of Innovation $(\mathrm{N}=102)$

Table 16. Multiple Linear Regression for Objective Facebook Indicators Predicting Perceptions of Innovation $(\mathrm{N}=102)$

\begin{tabular}{llllll}
\hline Objective Innovation Indicators & $\mathrm{B}$ & $\mathrm{SE}$ & $\beta$ & $t$ & $P$ \\
\hline
\end{tabular}




\begin{tabular}{lrrrrr} 
Frequency of word 'innovation' & .00 & .04 & .00 & .03 & .98 \\
Frequency of word 'risk' & .11 & .04 & .27 & 2.90 & .005 \\
$\begin{array}{l}\text { Frequency of word 'technology' } \\
\begin{array}{l}\text { Information about training/education } \\
\text { opportunities posted }\end{array}\end{array}$ & .01 & .04 & .02 & .20 & .84 \\
$\begin{array}{l}\text { Posts of advertisements for new } \\
\text { product/service launches }\end{array}$ & .16 & .04 & -.08 & -.85 & .40 \\
\hline
\end{tabular}

Note. $\mathrm{B}=$ unstandardized coefficient, $\mathrm{SE}=$ standard error, $\beta=$ standardized coefficient.

For the Dominance dimension, the model as a whole explained $21.1 \%$ of the variance in perceptions of Dominance, $F(3,98)=7.76, p<.001$. Of the three objective Facebook indicators, 'performance awards listed' was the only significant predictor of perceptions of Dominance $(\beta=.46, p<.001)$. Results suggest that higher instances of the word 'success' in company profile posts relates to higher perceptions of Dominance. See details in Table 17.

Table 17. Multiple Linear Regression for Objective Facebook Indicators Predicting Perceptions of Dominance $(\mathrm{N}=102)$

\begin{tabular}{lrrrrr}
\hline Objective Dominance Indicators & \multicolumn{1}{l}{ B } & \multicolumn{1}{l}{ SE } & \multicolumn{1}{l}{$l$} & \multicolumn{1}{l}{$P$} \\
\hline Awards for best places to work listed & .02 & .03 & .06 & .69 & .49 \\
Financial information listed & -.02 & .04 & -.05 & -.49 & .63 \\
Performance awards listed & .17 & .04 & .46 & 4.50 & $<.001$
\end{tabular}

Note. $\mathrm{B}=$ unstandardized coefficient, $\mathrm{SE}=$ standard error, $\beta=$ standardized coefficient.

For the Thrift dimension, the model as a whole explained $8.3 \%$ of the variance in perceptions of Thrift, $F(2,99)=4.49, p<.05$. However, neither of the two objective Facebook indicators showed significant relationships with perceptions of Thrift. See details in Table 18. However, preliminary analysis showed evidence of strong multicolinearity between the two indicators $(r=.60, p<.001)$ suggesting the two predictors should not be evaluated together. Therefore, evaluated separately, both 
'number of posts by page' $(\beta=-.27, p<.01)$ and 'total number of images/photos on profile' $(\beta=-.25, p<.05)$ were significantly, negatively related to perceptions of Thrift.

Table 18. Multiple Linear Regression for Objective Facebook Indicators Predicting Perceptions of Thrift $(\mathrm{N}=102)$

\begin{tabular}{llllll}
\hline Objective Thrift Indicators & \multicolumn{1}{l}{$\mathrm{B}$} & $\mathrm{SE}$ & $\beta$ & \multicolumn{1}{l}{$t$} \\
\hline Number of posts by page & -.07 & .04 & -.19 & -1.55 & .13 \\
Total number of images/ photos on profile & -.05 & .04 & -.13 & -1.11 & .27
\end{tabular}

Note. $\mathrm{B}=$ unstandardized coefficient, $\mathrm{SE}=$ standard error, $\beta=$ standardized coefficient.

Lastly, for the Style dimension, the model was not significant, $F(2,99)=1.91, p$

$=.15$. Nevertheless, see Table 19 for details.

Table 19. Multiple Linear Regression for Objective Facebook Indicators Predicting Perceptions of Style $(\mathrm{N}=102)$

\begin{tabular}{lccccc}
\hline Objective Style Indicators & B & SE & $\beta$ & $t$ & $p$ \\
\hline $\begin{array}{l}\text { Number of links to other [social] media } \\
\text { sites }\end{array}$ & .11 & .06 & .18 & 1.84 & .07 \\
$\begin{array}{l}\text { Number of posts containing } \\
\text { graphics/artwork }\end{array}$ & .03 & .06 & .05 & .50 & .62 \\
\hline
\end{tabular}

Note. $\mathrm{B}=$ unstandardized coefficient, $\mathrm{SE}=$ standard error, $\beta=$ standardized coefficient. 


\section{Chapter V. Discussion}

Personnel selection is a pivotal function of any organization. Core to this are the recruitment practices instilled by an organization. Recruitment is the process by which organizations attain the talent they need to be successful. The ability to recruit the best applicants not only eases the rest of the employee selection process, but ultimately benefits the organization as a whole by increasing personnel efficiency and reducing cost. More importantly, however, the inability to recruit the best applicants can lead to increased spending in selection systems, rising training and development expenditures, and high turnover rates.

Companies are increasingly turning to web-based recruitment methods to reach larger audiences in a fast and cost-effective manner. More so, researchers have found that job seekers use the information provided on recruitment websites to shape their perceptions of organizational image which then influences overall fit and attraction to organizations (Braddy et al., 2009; Dineen et al., 2002; Kroustalis, 2006). Unfortunately, limited research exists regarding how potential applicants form perceptions of image from web-based recruitment sources (e.g., Braddy et al., 2006; Braddy et al., 2009). In an effort to better inform organizations on how to attract the best applicants for the job, this study examined how perceptions of image are influenced through corporate websites and Facebook profiles, in the context of personnel recruitment.

In respect to Research Questions 1a-5a, the development and validation of objective website indicators yielded promising results. Of the initial set of 29 objective Website indicators theoretically derived, a total of 15 were retained through the content and empirical validation. It is important to note that some indicators were eliminated due 
to research limitations (e.g., lack of representation in the webpages, normality problems) and not due to conceptual reasons. Below is a summary of the website indicators found to predict each of the five organizational personality dimensions through the content and empirical validation.

Website indicators for the Boy Scout dimension:

- Frequency of word 'safety'

- Frequency of word 'support'

- Information about charity work posted

- Information about community involvement posted

- Information about employee benefits posted

- Presence of an employee recognition program

Website indicators for the Innovation dimension:

- Frequency of word 'innovation'

- Frequency of word 'technology'

Website indicators for the Dominance dimension:

- Awards for best places to work listed

- Financial information listed

- Frequency of word 'success'

Website indicators for the Thrift dimension:

- Frequency of word 'budget'

- Length of 'about us' section (reverse scored)

Website indicators for the Style dimension:

- Number of links to other [social] media sites

- Information about environmental-awareness listed

The confirmatory factor analysis which tested the five- factor structure of the website indicators showed good model fit, in line with the conceptual model of OP. However, despite the strong factor-structure of the website indicators, the convergent validity analysis between the objective website OP scores and the perceptions of OP score for the Website condition were largely non-significant. Although Thrift did yield a significant positive relationship across the two measures, a small effect size $(r=.20)$ provides minimal convergent support. While none of the other dimensions showed 
convergent validity, it is interesting to note that the objective Boy Scout score was negatively related to perceptions of Innovation $(r=-.26)$ and Style $(r=-.37)$ as well as positively related to perceptions of Thrift $(r=.28)$.

In light of the non-significant findings, convergent validity was also examined at the indicator level to explore whether or not specific components of the objective scores were more closely aligned with perceptions of OP. However, supplementary analyses at the individual indicator level produced similar results. Although this could be partially attributed to conceptual overlap between the dimensions, it is also possible that the content and empirical validation of the objective indicators resulted in restricted measurement of the full scope of each dimension. Alternatively, it is important to note that the confirmatory factor analysis for the validated measure of perceptions of OP yielded marginal results across the three experimental conditions (see Table ). This draws into question the construct validity of the measure and the five-factor conceptualization proposed by Slaughter et al. (2004).

Turning to Research Questions 1b-5b, the development and validation of objective Facebook indicators yielded promising results. Of the initial set of 38 objective Facebook indicators theoretically derived, a total of 20 were retained through the content and empirical validation - with one of the indicators representing two dimensions. As with the objective website indicators, some were eliminated due to issues with normality and not due to conceptual reasons. Given the wide variety of number of posts across company profiles-- and consequently data available to analyze - the range of values for some of the objective indicators created a naturally skewed pattern. Below is a summary 
of the Facebook indicators found to predict each of the five organizational personality dimensions through the content and empirical validation.

Facebook indicators for the Boy Scout dimension:

- Frequency of word 'respect'

- Information about charity work posted

- Information about community involvement posted

- Information about employee benefits posted

- Number of photos posted of people

- Information about continuing education posted

- Awards for best places to work posted

- Frequency of word 'diversity'

Facebook indicators for the Innovation dimension:

- Frequency of word 'innovation'

- Frequency of word 'risk'

- Frequency of word 'technology'

- Information about training/education opportunities posted

- Posts of advertisements for new product/service launches

Facebook indicators for the Dominance dimension:

- Awards for best places to work posted

- Postings relating to financial information

- Performance awards listed

Facebook indicators for the Thrift dimension:

- Number of posts by page (reverse scored)

- Total number of images/ photos on profile (reverse scored)

Facebook indicators for the Style dimension:

- Number of links to other [social] media sites

- Number of posts containing graphics/artwork

In terms of convergent validity for the Facebook objective scores, results were mainly as hypothesized. Specifically, convergent validity was present for the Dominance $(r=.37, p<.001)$ and Thrift $(r=.29, p<.01)$ dimensions, suggesting a moderate amount of overlap between the two measures. With marginally significant results, the Boy Scout $(r=.17, p<.10)$, Innovation $(r=.17, p<.10)$, and Style $(r=.17, p<.10)$ dimensions showed support in the direction of convergence. Worth noting, none of the objective 
scores displayed signs of convergence with perceptions of OP that were not specific to the hypothesized dimension. As with the objective website scores, more targeted convergent analyses were performed at the individual indicator level. Results from this supplementary analysis revealed stark convergent differences within the Facebook indicators. Despite the content and empirical validation process, select indicators for each dimension were revealed to be driving the convergence with perceptions of OP.

It is important to note, however, that the objective indicator confirmatory factor analysis which tested the five- factor structure of the Facebook indicators yielded marginally good results, compared to the Website indicators. While some fit indices suggested a good fitting model (i.e., RMSEA, CFI), other indices fell shy of the satisfactory threshold. Since the CFAs for perceptions of OP were also marginally good, taken together with the support for convergence, it is possible that both the objective and the validated measure are measuring the same criterion — but one that is not well supported by the five-factor model proposed by Slaughter at al. (2004).

Alternatively, the differing convergence results between the objective measures of $\mathrm{OP}$ and perceptions of OP could suggest fundamental differences in the two recruitment platforms. Company Facebook profiles allow for considerably more customization and a more dynamic means to communicate with job seekers than compared to traditional webpages. Facebook profiles may, by design, be more expressive and are able to more appropriately convey multi-dynamic OP dimensions. In line with media richness theory discussed earlier, this increased expressivity may play a major role in effectively projecting each of the OP dimensions. 
In addition to possible differences across the two web-based platforms, it is important to consider potential differences in terms of the observability of each OP dimension. As has been found in the individual personality assessment literature, certain Big Five personality traits, such as Emotional Stability, represent a more internal individual difference that is not as apparent to others, such as Agreeableness or Extraversion, that rely heavily on interaction with others. Consequently, these internallyfelt traits are less observable to others and more accurately measured through self-report methods. In line with this, it is probable that certain OP dimensions are less easily projected, and therefore observable, by job seekers. Such implications could make it difficult for potential job seekers to accurately rate certain dimensions over others. For example, the Style dimensions, by nature, is more visual and observable than its counterparts.

Hypotheses three and four posited that both perceptions of OP and objective scores of OP would differ depending on the recruitment source. In terms of perceptions of OP, results suggest that exposure to either of the recruitment materials - either through corporate websites or Facebook profiles-influenced perceptions of OP (except for the Dominance dimension), as compared to the group who was not exposed to any recruitment materials. Specifically, organizations were perceived as having significantly lower Boy Scout and Innovation levels when participants were not exposed to any recruitment material. The same companies, however, were perceived as being more Boy Scoutish and Innovative when participants were exposed to either the organization's corporate website or Facebook profile. In terms of Innovation, it seems rational that companies who embrace more modern web-based methods of recruitment are inherently 
perceived as being more innovative through their adoption of new technology. The use of web-based media also allows organizations to convey information in rich media formats that can be both informative and appealing. In terms of elevated Boy Scout perceptions, it is likely the mere effect of the organization relaying information to potential applicants is perceived favorably in terms of valuing and caring about interaction with potential applicants.

In line with this rational, organizations were viewed as being significantly more Thrifty by participants not exposed to web-based recruitment material, as compared to those who were. Given that Thrifty companies are characterized as being simple, economical, and even sloppy, it is possible that mere exposure to new recruitment material increases the applicant's perception of the company in terms of financial resources for recruitment.

When it comes to the Style dimension, organizations were perceived as being significantly more Stylish when participants were exposed to the company's Facebook profile, as compared to the corporate website or no recruitment material. Based on Slaughter et al. (2007) conceptualization, Stylish organizations are described as trendy and hip. Given that Facebook is a relatively new, but quickly growing, method of web based recruitment, it suggests that organizations who adopt this method are viewed as being more cool and trendy.

Since a large portion of organizations $(\mathrm{N}=52)$ were omitted due to insufficient data in the No Exposure condition, supplementary analysis was also performed to assess differences in just Website and Facebook perceptions using the full set of organizations $(\mathrm{N}=102)$. Although replication of the significant findings between no exposure and any 
exposure (website or Facebook) was not an option, results supported original results suggesting organizations are perceived as being more Stylish after exposure to Facebook profile materials than Website. Interestingly, supplementary analyses also revealed that organizations were viewed as more Innovative after exposure to Facebook profile materials, than compared to exposure to Website materials. Given the relatively novel practice of using social media websites to interact with potential applicants, organizations who adopt new tools are seen as more advanced and 'cutting edge.' As previously mentioned, it is possible that a lack of power contributed to this non-significant finding using the smaller sub-set of organizations.

Although not included in any of the hypotheses or research questions, measures of organizational prestige and rank (i.e., Fortune 500 company ranking) were also collected. Previous research (e.g., Williamson et al., 2010) indicates that applicants who are exposed to recruitment materials for organizations with high levels of prestige may not be influenced as heavily as when they view recruitment materials for low prestige organizations. Organizations with high prestige are also likely to have very salient images already by the general public —images that likely would not change much regardless of exposure to additional material. As supplementary exploratory analysis, this study examined whether or not the divergent findings remained when considering the effect of prestige. In other words, do perceptions of OP vary across conditions differently based on varying levels of organizational prestige? Prestige was assessed using both subjective student reported levels of prestige (subsequently aggregated consistent with other variables) and objective ranks based on the Fortune 500 listing. Surprisingly, neither prestige nor rank showed significant effect on changes in OP across the three 
experimental conditions. Findings imply that exposure to recruitment materials does influence perceptions of OP regardless of an organizations level of prestige or rank.

Given the differing perceptions of OP across the experimental conditions, it was also expected that the objective indicators of OP would display similar results. Due to the non-normality of the objectives indicators, scores were standardized (i.e., z-scored) prior to any analysis, therefore eliminating the option of testing for group differences between the two recruitment methods. Nevertheless, the linear relationship was examined in a method consistent with divergent validity analysis.

Consistent with the results obtained from hypothesis three, results indicated a non-significant relationship between the two objective Style scores $(r=-.01, p=.94)$ thus providing evidence for divergence. It is important to note, however, that correlation analysis does not provide information regarding which recruitment method yielded higher perceptions of Style. For the Innovation dimension, results indicated a large, positive relationship between the two objective scores $(r=.49, p<.001)$ offering little evidence for divergent validity. Given the limitations of correlation analysis, it is possible that although the scores are highly correlated, that consistent mean differences still exist between the two scores. The Thrift dimension also indicated a moderate, positive effect $(r=.32, p<.01)$ suggesting that the two objective scores are partially related. Neither the Boy Scout nor the Dominance dimension analyses yielded significant relationships signifying little to no similarity. As will be discussed below, future research would greatly benefit from objective indicators that are amenable to mean difference analyses to fully understand the relationship between the two recruitment sources. 
The final goal of this study was to identify which components of corporate websites and company Facebook profiles have the most impact on perceptions of image. In order to examine the relative contribution of each objective indicator on perceptions of OP, the predictive validity of each subset of indicators (e.g., Boy Scout objective indicators, Innovation objective indicators, etc.) was examined using regression analyses.

Unfortunately, as foreshadowed by the convergent analysis, none of the objective indicators were shown to significantly predict perceptions of OP. In addition to issues surrounding the development of the objective website indicators, a possible explanation for this could lie in construct validity of the validated OP measure.

Results from the predictive analysis of the Facebook indicators, on the other hand, yielded fruitful outcomes. Each of the OP dimensions appeared to be highly influenced by one or two objective indicators. Analyses revealed that organizations perceived as high in the Boy Scout dimension had a greater number of posts of employees or customers on their Facebook profile. Similarly, these organizations also used the word 'diversity' abundantly in their postings. For organizations high in the Innovation dimension, profiles would frequently advertise new products or services as well as a routine of using of the word 'risk.' Alternatively, organizations with high perceptions of Dominance were related to high occurrences of the word 'success.' In line with characteristics of Thrift, these organizations had the lowest number of company-initiated posts as well as the least number of photos associated with their profile. Lastly, organizations perceived as Stylish were characterized by having numerous links to other social media websites listed on their Facebook profile. A more detailed discussion of the implications of these findings is available below. 


\section{Practical Implications}

Findings from this study have profound implications for the field of personnel recruitment. With a growing number of organizations embracing web-based recruitment methods, it is essential that organizations have the necessary knowledge to manage recruitment efforts efficiently. This research provides clear guidelines to help organizations better streamline their image on web-based recruitment sources, namely corporate websites and company-run Facebook profile pages.

One of the most pivotal findings is that organizations are not presenting consistent image projections through their various recruitment sources. The idea that organizations are being inconsistent with their image projections was supported by the varying perceptions of image depending on exposure to website pages or Facebook profiles and also by the objective components of the web pages. This research suggests that the mere use of web-based recruitment sources increases the public's perception of the company in terms of Innovation and Boy Scout. More so, organizations that employ Facebook profile pages as means of recruiting employees are also perceived as more trendy and stylish. These implications are pivotal for organizations that want to project a culture of Innovation, Boy Scout, or Style.

It is possible that organizations are intentionally driving different image perceptions in an attempt to appeal to different applicant pools. However, given potential applicant's ease of accessibility to multiple web-based recruitment sources, it is important that organizations project a consistent image so as not to confuse applicants with inconsistent information. Organizations should determine whether it is beneficial to create different image profiles on each recruitment platform. This may be particularly 
useful when trying to appeal to a younger demographic of potential applicants such as the Millennials or Generation Z. (Macky, Gardner, \& Forsyth, 2008; Twenge \& Campbell, 2008).

Findings from this study also begin to inform organizations on how perceptions of image are formed by corporate websites and company Facebook profiles. By identifying specific characteristics of webpages that impact perceptions of image, organizations are now better able to tailor their recruitment media to maximize efficiency. As mentioned above, content on corporate websites appears to have less impact on perceptions of image, compared to content on Facebook profiles. It is possible that corporate websites are now viewed as less novel, and consequently less mental effort is spent internalizing the content, compared to the more innovative web-based platforms such as Facebook. Nevertheless, some website features were found to be related to specific image dimensions. Occurrences of the words 'safety' and 'support,' for example, were related to perceptions of Thrift, whereas the absence of those words was found to be related to perceptions of Innovation. Table 20 shows a summary of the key website objective components. Turning to company Facebook profiles, several features were found to be related to specific image dimensions. For example, organizations that want to project an image consistent with the Boy Scout dimension should post frequent pictures of their employees and customers. Alternatively, organizations who pride themselves on innovation should make it a priority to share news about new products of services on their Facebook profile. Table 20 shows a summary of the key Facebook objective components. 
Table 20. Key Objective Components Related to Image for Website and Facebook

$$
\text { Related Objective Components }
$$

\begin{tabular}{|c|c|c|}
\hline OP Dimension & Corporate Website & Company Facebook Profiles \\
\hline Boy Scout & & $\begin{array}{ll}\text { - } & \text { Presence of photos of people } \\
\text { - } & \text { Use of the word 'diversity' }\end{array}$ \\
\hline Innovation & $\begin{array}{l}\text { - Absence of the word } \\
\text { 'support'* } \\
\text { - Absence of information } \\
\text { related to community } \\
\text { involvement* }\end{array}$ & $\begin{array}{ll}\text { - } & \text { Information about new } \\
\text { products or services } \\
\text { - } & \text { Use of the word 'risk' } \\
\text { - } & \text { Information about } \\
\text { performance awards } \\
\text { received* } \\
\text { - } & \text { Total number of posts* } \\
\text { - } & \text { Total number of } \\
& \text { image/photos* }\end{array}$ \\
\hline Dominance & & $\begin{array}{ll}\text { - } & \text { Use of the word 'success' } \\
\text { - } & \text { Information about new } \\
\text { products or services* } \\
\text { - } & \text { Total number of posts* } \\
\text { - } & \text { Links to other social media } \\
\text { sites* }\end{array}$ \\
\hline Thrift & $\begin{array}{l}\text { - Use of the word 'safety'* } \\
\text { - Use of the word } \\
\text { 'support'* } \\
\text { - Information relating to } \\
\text { community involvement* }\end{array}$ & $\begin{array}{ll}\text { - } & \text { Lack of total posts } \\
\text { - } & \text { Lack of total images/photos } \\
\text { - } & \text { Absence of financial data* } \\
\text { - } & \text { Absence of information } \\
& \text { about awards received* } \\
\text { - } & \text { Absence of information } \\
\text { about new products or } \\
\text { services* }\end{array}$ \\
\hline Style & $\begin{array}{l}\text { - Absence of the word } \\
\text { 'safety'* } \\
\text { - Absence of the word } \\
\text { 'support'* } \\
\text { - Absence of information } \\
\text { related to community } \\
\text { involvement* }\end{array}$ & $\begin{array}{ll}\text { - } & \text { Links to other social media } \\
\text { sites } \\
\text { - } & \text { Total number of posts* } \\
\text { - } & \text { Total number of } \\
\text { image/photos* } \\
\text { - } \\
\text { Information about new } \\
\text { products or services* } \\
\text { - } & \text { Use of the word 'diversity' }\end{array}$ \\
\hline
\end{tabular}

Note. OP = Organizational Personality

* Indicates a component not originally hypothesized to predict respective OP dimension.

It is important for organizations to view new web-based recruitment sources as a beneficial resource and not as a frivolous addition to recruitment efforts. Social media 
sites, such as Facebook and Twitter, allow for richer-media and a higher level of organization-applicant interaction than traditional recruitment methods. The new platforms enable organizations to relay complex and dynamic information much better than they ever could before. Moreover, organizations that are slow to embrace these new platforms, or that choose to refrain from using the resource altogether, may be unknowingly projecting an unwanted image due to their lack of involvement.

\section{Limitations}

There were a number of limitations that were encountered throughout the duration

of the study. In order to adhere to a standardized method of selecting companies without bias or prejudices, companies were selected from the Fortune 5002013 publicly available list. Considering the time consuming task of gathering and coding relevant information for each company's corporate Website and Facebook profile page, the number of organizations decided upon for inclusion was limited by the feasibility in gathering all of the data in a reasonable amount of time so as to avoid history or maturation biases. Care should be taken to consider the generalizability of the current company sample to other organizations.

Companies were selected for assessment by systematically picking companies in descending order from 500 that fit the inclusion criteria. Although this method of selecting companies was chosen to minimize the expected effect of prestige on the malleability of organizational image, this method inherently limited the range of variability in organizational prestige. Additionally, this contributed an unexpectedly small No Exposure condition because of the fact that many of the student participants were not familiar with some organizations, and consequently were not able to report their 
perceptions of image for the target organization. Although this was not a limitation in the Website and Facebook condition since those student participants were able to provide image ratings after being exposed to recruitment material, future research should aim to have more equal sample sizes across all conditions. Nevertheless, despite the substantially smaller No Exposure group, results provided support for varying perceptions of image.

Turning to the objective indicator analysis, one of the clear limitations in the data analysis stemmed from the lack of observable data for the objective Website and Facebook indicators. For the corporate website, it was decided to only measure indicators present in the 'careers' homepage as well as the 'about us' page. This was done in order to both standardize the method for selecting content across organizations, and also since are the two pages potential applicants are most likely to be exposed to. Although many of the organizations provided links to subsections of the careers page, it could not be assumed that all potential applicants would exhaust the full amount of links within that section. As a result, the amount of content available for analysis was significantly smaller than compared to the amount of content available for 30 days' worth of Facebook profile activity. For some of the indicators, this contributed to the low, or non-existent, occurrence counts of data points (i.e., number of time a particular word was noted, the presence of music, etc.). Therefore, some of the objective indicators that were content and empirically validated could not be included in subsequent analyses due to a lack of variability. Although a lack of variability was less of a problem for the Facebook profile indicators, this portion of analyses suffered from extreme outliers for select indicators. For example, for 'number of people talking about the company' a handful of 
organizations had values above 1 million, while most had values around 5,000. Although this data could have been useful in differentiating organizations, the outliers created extremely skewed data which removed the option of analyzing the data with parametric methods.

The objective indicator validation was also substantially limited by the inability to cross-validate the factor structure supported by the empirical validation. Since the number of indicators representing to each dimension was refined in the process of performing the confirmatory factor analysis, the analysis was also exploratory in nature. Although the indicators provided low factor loadings for this set of organizations, it is important that the structure be cross-validated with a second set of equivalent organizations. Doing so would truly offer support for the generalizability of the findings to other organizations.

Lastly, it should be noted that although Slaughter et al.'s (2007) measure of perceptions of organizational personality has been validated, the three confirmatory factor analyses did not yield particularly favorable fit statistics. The weak CFA structure obtained calls into question the factor structure of the model overall, which has profound implications for the factor structure expected for the objective indicators as well. It is possible that the student sample used for this study played a role in the lackluster psychometric properties of the factor structure.

\section{Future Directions}

This study serves to show how the underlying components of corporate webpages and Facebook profiles influence perceptions of organizational image. As mentioned above, additional research on this topic would be useful to verify the generalizability of 
these findings to other companies. This is particularly important in terms of further identifying the objective indicators of personality. Not only would it be useful to crossvalidate the objective indicators in other similar organizations, but also to organizations not represented in the Fortune 500 ranking (e.g., non-profit organizations, internationally headquartered organizations). Related to generalizability of the objective indicator findings, it would be fruitful to examine if organizations established as strongly representing a particular dimension display the objective indicators identified through this research. For example, Apple is considered a highly Innovative company and therefore should display the objective indicators linked to Innovation on their corporate websites and Facebook profile. An alternative way to examine this is through experimental manipulation of fictitious organizational recruitment pages in a controlled laboratory setting. For example, does manipulation of the set objective indicators lead to differences in perceptions of $\mathrm{OP}$ as expected?

Future research should also strive to identify a more comprehensive list of all possible objective indicators for both recruitment platforms. An expanded list of indicators would be particularly useful for corporate websites given the less than favorable objective indicator results. In order to accomplish this, researchers could examine best practices in the marketing industry in terms of identifying possible objective components that could be impacting OP perceptions. In addition, many organizations already collect a great deal of data, commonly referred to as "big data" in the organizational behavior literature. Such data sets commonly include variables relating to website activity and performance, which could be a low-cost method of identifying possible objective indicators related to organizational image. Ultimately, this 
list could move towards a more comprehensive taxonomy of how objective web-based indicators represent each of the five OP dimensions. Going forward, it may be useful to take a more holistic approach by not only looking at each dimension in specific, but also the overall pattern created by the five dimensions together. By examining the collective OP dimensions, organizations can be assessed in terms of their overall OP profile in addition to just the implications of specific levels of each individual dimension. Analyses at this level would allow researchers to explore possible meaningful relationships across multiple OP dimensions.

Current research suggests that organizations with high prestige may be less influenced by content in their recruitment materials. For example, organizations such as Apple who are viewed as very prestigious, also tend to have very salient image perceptions already established in the general public. For organizations such as Apple, potential applicants are less likely to be influenced by content on recruitment sources. Although results did not reveal prestige as a confounding factor in the present study, additional research should examine this further. Also, since the sample of organizations was taken from the Fortune 5002013 rankings, all of the organizations had relatively positive prestige ratings. Future research should strive to also include organizations that have more questionable reputations (e.g., Phillip Morris, BP). Similarly, other factors that may be involved in the influential strength of recruitment content, such as familiarity with technology and credibility of sources, should be explored.

Looking further, it is essential that researchers continue to explore the intricacies of new web-based platforms as a recruitment tool. Technology continues to evolve, and so do the tools available for organizations to interact with potential applicants. 
Organizations must be informed in order to maximize the success of new recruitment tools. Even if web-based recruitment sources shift away from websites and social media sites towards newer and shinier recruitment alternatives, the underlying message that organizations are relaying regarding their image stays the same. As technology changes, organizations should strive to keep up with the underlying mechanisms of how new recruitment sources are shaping perceptions of image.

\section{Conclusion}

In sum, this study examined the role of corporate websites and company Facebook profiles in shaping perceptions of organizational image in the recruitment context. In addition to assessing differences in perceptions of image based on exposure to different recruitment sources, the study also examined the mechanisms through which perceptions of organizational image are influenced through website and profile content. Results indicate that exposure to corporate website and company Facebook profiles do influence perceptions of image, at times in different ways. Furthermore, individual components of the websites were identified as key drivers for influencing specific image dimensions, particularly for company Facebook pages. Findings are beneficial for advising practitioners on how to best manage their web-based recruitment sources in order to maximize efficiency. Although additional research on this topic is warranted, this study serves to further our understanding of the process through which perceptions of organizational image are influenced by new recruitment sources. 


\section{REFERENCES}

Aaker, J.L. (1997). Dimensions of brand personality. Journal of Marketing Research, 34, 347-356.

Albert, S., \& Whetten, D. A. (1985). Organizational identity. Research in Organizational Behavior; Research in Organizational Behavior.

Allen, D.G., Mahto, R.V., \& Otondo, R.F. (2007). Web-based recruitment: Effects of information, organizational brand, and attitudes toward a Web site on applicant attraction. Journal of Applied Psychology, 92, 1696-1708.

Ambler, T., \& Barrow, S. (1996). The employer brand. Journal of Brand Management, 4, 185-206.

Amichai-Hamburger, Y., \& Vinitzky, G. (2010). Social network use and personality. Computers in Human Behavior, 26(6), 1289-1295.

Anderson, N. (2003). Applicant and Recruiter Reactions to New Technology in Selection: A Critical Review and Agenda for Future Research. International Journal of Selection and Assessment, 11, 121-136.

Ashforth, B.E. \& Mael, F. (1989). Social identity theory and the organization, Academy of Management Review, 14, 20-39.

Back, M. D., Stopfer, J. M., Vazire, S., Gaddis, S., Schmukle, S. C., Egloff, B., \& Gosling, S. D. (2010). Facebook profiles reflect actual personality, not selfidealization. Psychological Science, 21(3), 372-374.

Backhaus, K., \& Tikoo, S. (2004). Conceptualizing and researching employer branding. Career development international, 9(5), 501-517.

Barber, A.E. (1998). Recruiting Employees: Individual and organizational perspectives. Thousand Oaks, CA: Sage Publications.

Barnes, N. G., Lescault, A. M., \& Andonian, J. (2012). Social media surge by the 2012 Fortune 500: Increase use of blogs, Facebook, Twitter and more. Charlton College of Business Center for Marketing Research.

Barnett, M., Jermier, J., \& Lafferty, B. (2006). Corporate reputation: The definitional landscape. Corporate Reputation Review, 9(1).

Belt, J. A., \& Paolillo, J. G. P. (1982). The influence of corporate image and specificity of candidate qualifications on response to recruitment advertisements. Journal of Management, 8, 105-112. 
Berg, P.O. (1985). Organization change as a symbolic transformation process. In P. Frost, L. Moore, M. R. Louis, C. Lundberg, \& J. Martin (Eds.), Reframing Organizational Culture, pp. 281-300. Beverly Hills, CA: Sage.

Bernstein, D. (1984) Company image and reality: A critique of corporate communications. Eastbourne, UK: Holt, Rinehart \& Winston.

Billsberry, J. (2007). Attracting for values: An empirical study of ASA's attraction proposition. Journal of Managerial Psychology, 22, 132-149.

Braddy, P. W., Meade, A. W., Michael, J. J., \& Fleenor, J W. (2009). Internet recruiting: Effects of website content features on viewers' perceptions of organizational culture. International Journal of Selection and Assessment, 17, 19- 34.

Braddy, P. W., Thompson, L. F., Wuensch, K. L., \& Grossnickle, W. G. (2003). Internet recruiting: The effects of web page design features. Social Science Computer Review, 21, 374-385.

Braddy, P.W., Meade, A.W., \& Kroustalis, C.M. (2006). Organizational Recruitment Website Effects on Viewers' Perceptions of Organizational Culture. Journal of Business and Psychology, 20, 525-543.

Breaugh, J. A., Greising, L. A., Taggart, J. W., \& Chen, H. (2003). The Relationship of Recruiting Sources and Pre-Hire Outcomes: Examination of Yield Ratios and Applicant Quality. Journal of Applied Social Psychology, 33(11), 2267-2287.

Breaugh, J. A., Macan, T. H., \& Grambow, D. M. (2008). Employee recruitment: Current knowledge and directions for future research. In G. P. Hodgkinson \& J. K. FordEds.), International Review of Industrial and Organizational Psychology, vol. 23. (pp. 45-82)New York: John Wiley \& Sons.

Breaugh, J.A. \& Starke, M. (2000). Research on employee recruitment: So many studies, so many remaining questions. Journal of Management, 26, 405-434.

Bretz, R.D., \& Judge, T.A. (1994). The role of human resource systems in job applicant decision processes. Journal of Management, 20, 531-551.

Brown, T. A. 2006. Confirmatory Factor Analysis for Applied Research. New York, NY: The Guilford Press.

Byrne, D.E. (1971). The Attraction Paradigm, New York: Academic Press.

Cable, D. M., \& Turban, D. B. (2003). The value of organizational reputation in the recruitment context: A brand equity perspective. Journal of Applied Social Psychology, 33, 2244-2266.

Capowski, G. (1997). Dealing with the labor shortage from the inside out. HR Focus, 74, 2. 
Cappelli, P. (2001). Making the most of on-line recruiting. Harvard Business Review, 79, $139-146$.

Carlson, K.D., Connerley, M.L., \& Mecham, R.L. (2002). Recruitment evaluation: The case for assessing the quality of applicants attracted. Personnel Psychology, 55, 461-490.

Cascio. W.F. \& Aguinis, H.E. (2005). Applied Psychology in Human Resource Management. (6th Edition). New York, NY: Prentice-Hall.

Chapman, D.S., \& Webster, J. (2003). The Use of Technologies in the Recruiting, Screening, and Selection Processes for Job Candidates. International Journal of Selection and Assessment, 11, 113-120.

Chapman, D.S., Uggerslev, K.L., Carroll, S.A., Piasentin, K.A. \& Jones, D.A. (2005). Applicant attraction to organizations and job choice: A meta-analytic review of the correlates of recruiting outcomes. Journal of Applied Psychology, 90, 928944.

Chatman, J. A., \& Jehn, K. A. (1994). Assessing the relationship between industry characteristics and organizational culture: how different can you be? Academy of management journal, 522-553.

Chen, C. C., Lin, M. M., \& Chen, C. M. (2012). Exploring the mechanisms of the relationship between website characteristics and organizational attraction. The International Journal of Human Resource Management, 23(4), 867-885.

Christiansen, N., Villanova, P., \& Mikulay, S. (1999). Political influence compatibility: Fitting the person to the climate. Journal of Organizational Behavior, 18(6), 709730 .

Cober, R. T., Brown, D. J., Blumental, A. J., Doverspike, D., \& Levy, P. (2000). The quest for the qualified job surfer: It's time the public sector catches the wave. Public Personnel Management, 29(4), 479-496.

Cober, R. T., Brown, D. J., Keeping, L. M., \& Levy, P. E. (2004). Recruitment on the net: How do organizational web site characteristics influence applicant attraction? Journal of Management, 30, 623-646.

Cober, R. T., Brown, D. J., Levy, P. E., Cober, A. B., \& Keeping, L. M. (2003). Organizational web sites: Web site content and style as determinants of organizational attraction. International Journal of Selection and Assessment, 11, 158-169.

Cooper, D., Robertson, I.T., \& Tinline, G. (2003). Recruitment and Selection: A framework for success. London: England, Thompson. 
Crispin, G., \& Mehler, M. (1997). Recruiting rockets through cyberspace. $H R$ Magazine, 42(12), 72-77.

Daft, R. L., \& Lengel, R. H. (1986). Organizational information requirements, media richness and structural design. Management science, 32(5), 554-571.

Davison, H. K., Maraist, C., \& Bing, M. N. (2011). Friend or foe? The promise and pitfalls of using social networking sites for HR decisions. Journal of Business and Psychology, 26(2), 153-159.

Deephouse, D. L. (2000). Media reputation as a strategic resource: An integration of mass communication and resource-based theories. Journal of management, 26(6), 1091-1112.

Devendorf, S. A., \& Highhouse, S. (2008). Applicant-employee similarity and attraction to an employer. Journal of occupational and organizational psychology, 81(4), 607-617.

Dineen, B. R., Ash, S. R., \& Noe, R. A. (2002). A web of applicant attraction: Personorganizational fit in the context of web-based recruitment. Journal of Applied Psychology, 87(4), 723-734.

Dineen, B.R., Ling, J., Ash, S.R., \& DelVecchio, D. (2007). Aesthetic Properties and Message Customization: Navigating the Dark Side of Web Recruitment. Journal of Applied Psychology, 92, 356-372.

Dutton, J. E., Dukerich, J. M., \& Harquail, C. V. (1994). Organizational images and member identification. Administrative Science Quarterly, 39, 239-263.

Dutton, J.E. and Dukerich, J.M. (1991), Keeping an eye on the mirror: image and identity in organizational adaptation. Academy of Management Journal, 34, pp. 517-54.

Erikson, M. K. (1996). Using Self-congruity and Ideal Congruity to Predict Purchase Intentions: A European Perspective. Journal of Euro-Marketing, 6(1), 41-56.

Facebook (2013) News Press. Retrieved from http://www. facebook.com.

Fernandez, R. M., \& Weinberg, N. (1997). Sifting and sorting: Personal contacts and hiring in a retail bank. American Sociological Review, 883-902.

Fombrun, C. J. (1996). Reputation: Realizing value from the corporate image. Boston: Harvard Business School Press.

Fombrun, C., \& Riel, C. V. (1997). The reputational landscape. Corporate Reputation Review, 1-16. 
Foti, R. J., \& Lord, R. G. (1987). Prototypes and scripts: The effects of alternative methods of processing information on rating accuracy. Organizational Behavior and Human Decision Processes, 39(3), 318-340.

Funder, D. C. (1995). On the accuracy of personality judgment: a realistic approach. Psychological Review, 102(4), 652.

Gatewood, R. D., Gowan, M. A., \& Lautenschlager, G. J. (1993). Corporate image, recruitment image, and initial job choice decisions. Academy of Management Journal, 36, 414-427.

Ginzel, L. E., Kramer, R. M., \& Sutton, R. I. (1993). Organizational impression management as a reciprocal influence process: The neglected role of the organizational audience. Research in Organizational Behavior, 15, 227-227.

Gioia, D. A., \& Thomas, J. B. (1996). Identity, image, and issue interpretation: Sensemaking during strategic change in academia. Administrative science quarterly, 370-403.

Gioia, D. A., Schultz, M., \& Corley, K. G. (2000). Organizational identity, image and adaptive instability. Academy of Management Review, 25, 63-81.

Goldberg, C. B., \& Allen, D. G. (2008). Black and white and read all over: Race differences in reactions to recruitment Web sites. Human Resource Management, $47(2), 217-236$.

Goltz, S. M., \& Giannantonio, C. M. (1995). Recruiter friendliness and attraction to the job: The mediating role of inferences about the organization. Journal of Vocational Behavior, 46(1), 109-118.

Gregory, P. (2010). Assessing the influence of organizational personality, applicants' need motivation, expectancy beliefs, and person-organization fit on applicant attraction. (Unpublished doctoral dissertation). Florida International University, FL.

Gregory, P. J. \& Viswesvaran, C. (2009). A tale of two attributions: The relative importance of image to attraction. Poster session presented at the annual meeting of the Society for Industrial and Organizational Psychology, New Orleans, LA.

Grunig, J. E. (1993). Image and substance: From symbolic to behavioral relationships. Public Relations Review, 19(2), 121-139.

Haefner, R. (2009). More employers screening candidates via social networking websites. Retrieved December 3, 2009, from http://msn.careerbuilder. com/Article/MSN-2035-Job-Info-and-Trends-Mo?ArticleID=2035\& cbRecursionCnt $=1 \&$ cbsid $=6 c c 42 \mathrm{e} 9 \mathrm{a} 7 \mathrm{~d} 8 \mathrm{~b} 43 \mathrm{bbab} 1736 f 6893 \mathrm{a} 4 \mathrm{dfc}-313167165$ w0-6 
Hatch, M. J., \& Schultz, M. (1997). Relations between organizational culture, identity and image. European Journal of marketing, 31(5/6), 356-365.

Havenstein, H. (2008). One in five employers uses social networks in hiring process. Retrieved June, 18, 2009.

Highhouse, S., Thornbury, E. E., \& Little, I. S. (2007). Social-identity functions of attraction to organizations. Organizational Behavior and Human Decision Processes, 103, 134-146.

Hogan, R. T. (1991). Personality and personality measurement. In M. D. Dunnette, \& L. M. Hough (Eds.), Handbook of industrial and organizational psychology, vol. 2 (2nd ed.). (pp. 873-919). Palo Alto, CA, US: Consulting Psychologists Press.

Howard, J. A., \& Sheth, J. N. (1969) The Theory of Buyer Behavior. Appleton-CenturyCrofts, New York, NY.

Hu, C., Su, H.C., \& Chen, C.I.B. (2007), The Effect Of P-O Fit Feedback Via Recruitment Web Site on Applicant Attraction. Computer in Human Behavior, 23, 2509-2523.

Jackson, S. (1992). Message effects research: Principles and design of analysis. New York: Guilford.

Judge, T. A., \& Bretz, R.D. (1992). Effects of work values on job choice decisions. Journal of Applied Psychology, 79, 937-949.

Judge, T. A., \& Cable, D. M. (1997). Applicant personality, organizational culture, and organization attraction. Personnel Psychology, 50, 359-394. Cable, D. M., \& Judge, T.A. (1997). Interviewer's perceptions of person-organization fit and organizational selection decisions. Journal of Applied Psychology, 82, 546-561.

Jue, A. L., Marr, J. A., \& Kassotakis, M. E. (2009). Social media at work: How networking tools propel organizational performance. Jossey-Bass.

Kaplan, A., \& Haenlein, M. (2010). Users of the world, unite! The challenges and opportunities of social media. Business Horizons, 53(1), 59-68.

Karl, K., Peluchette, J., \& Schlaegel, C. (2010). Who's Posting Facebook Faux Pas? A Cross-Cultural Examination of Personality Differences. International Journal of Selection and Assessment, 18(2), 174-186.

Kluemper, D. H., Rosen, P. A., \& Mossholder, K. W. (2012). Social Networking Websites, Personality Ratings, and the Organizational Context: More Than Meets the Eye? Journal of Applied Social Psychology, 1143-1172. 
Kristof, A. L. (1996). Person-organization fit: An integrative review of its conceptualizations, measurement, and implications. Personnel Psychology, 49, 149.

Kristof-Brown, A.L., Zimmerman, R.D., \& Johnson, E.C. (2005). Consequences of Individuals' Fit at Work: A Meta-Analysis of Person-Job, Person-Organization, Person-Group, and Person- Supervisor Fit. Personnel Psychology, 58, 281-342.

Laroche, M., Habibi, M. R., Richard, M. O., \& Sankaranarayanan, R. (2012). The effects of social media based brand communities on brand community markers, value creation practices, brand trust and brand loyalty. Computers in Human Behavior, $1-13$.

Leong, E. K., Stanners, P. J., \& Huang, X. (1998). Web Site Objectives: Perspective from Western Australian Companies. In ANZMAC 1998 Conference Proceedings (on CD), University of Otago, Dunedin, New Zealand, Nov/Dec.

Lievens, F. (2006). Organizational Image/reputation. In. S. Rogelberg \& C.L. Reeve (Eds.) The Encyclopedia of Industrial and Organizational Psychology (pp.568570). New York: Sage.

Lievens, F., \& Highhouse, S. (2003). The relation of symbolic and instrumental attributes to company's attractiveness as an employer. Personnel Psychology, 56, 75-102.

Lievens, F., Decaesteker, C., Coetsier, P., \& Geirnaert, J. (2001). Organizational attractiveness for prospective applicants: A person-organisation fit perspective. Applied Psychology, 50(1), 30-51.

Lievens, F., van Dam, K., \& Anderson, N. (2002). Recent trends and challenges in personnel selection. Personnel Review, 31(5), 580-601.

Lievens, F., Van Hoye, G., \& Anseel, F. (2007). Organizational identity and employer image: Towards a unifying framework. British Journal of Management, 18, S45S59.

Llorens, J. J., \& Kellough, J. E. (2007). A revolution in public personnel administration: The growth of web-based recruitment and selection processes in the federal service. Public Personnel Management, 36(3), 207.

Lloyd, S. (2002). Branding from the inside out. Business Review Weekly,24(10), 64-66.

Lyons, B. D., \& Marler, J. H. (2011). Got image? Examining organizational image in web recruitment. Journal of managerial psychology, 26(1), 58-76.

Markus, H., \& Wurf, E. (1987). The dynamic self-concept: A social psychological perspective. Annual review of psychology, 38(1), 299-337. 
Maurer, S. D., \& Liu, Y. (2007). Developing effective e-recruiting websites: Insights for managers from marketers. Business horizons, 50(4), 305-314.

Maurer, S. D., Howe, V., \& Lee, T. W. (1992). Organizational recruiting as marketing management: An interdisciplinary study of engineering graduates. Personnel Psychology, 45(4), 807-833.

Muniz Jr, A. M., \& O'guinn, T. C. (2001). Brand community. Journal of consumer research, 27(4), 412-432.

Nielson.com (2009) Personal Recommendations And Consumer Opinions Posted Online Are The Most Trusted Forms Of Advertising Globally. Retrieved from http://blog.nielsen.com/nielsenwire/wp-content/uploads/2009/07/pr_globalstudy_07709.pdf.

Nolan, K. P., \& Harold, C. M. (2010). Fit with what? The influence of multiple self-concept images on organizational attraction. Journal of Occupational and Organizational Psychology, 83(3), 645-662.

Norman, W. T., \& Goldberg, L. R. (1966). Raters, ratees, and randomness in personality structure. Journal of Personality and Social Psychology; Journal of Personality and Social Psychology, 4(6), 681.

Olins, W. (1989), Corporate Identity: Making Business Strategy Visible through Design, Thames and Hudson, London.

Olins, W. (1995). The new guide to identity. Aldershot: Gower.

O'Reilly, C. A., Chatman, J., \& Caldwell, D. F. (1991). People and organizational culture: A profile comparison approach to assessing person-organization fit. Academy of Management Journal, 34, 487-516.

Park, C. W., Jaworski, B. J., \& Maclnnis, D. J. (1986). Strategic brand concept-image management. The Journal of Marketing, 135-145.

Pendergrast, M. (1993) For God, Country and Coca-Cola. New York: Charles Scribner's Sons.

Plummer, J. T. (1985). How personality makes a difference. J Adv Res; 24(6), 27-31.

Popovich, P., \& Wanous, J. P. (1982). The realistic job preview as a persuasive communication. Academy of Management Review, 570-578.

Powell, G. N., \& Goulet, L. R. (1996). Recruiters' and applicants' reactions to campus interviews and employment decisions. Academy of Management Journal, 39, 1619-1640. 
Quercia, D., Kosinski, M., Stillwell, D., \& Crowcroft, J. (2011, October). Our twitter profiles, our selves: Predicting personality with twitter. In Privacy, security, risk and trust (passat), 2011 ieee third international conference on and 2011 ieee third international conference on social computing (socialcom) (pp. 180-185). IEEE.

Rafaeli, A., Hadomi, O., \& Simons, T. (2005). Recruiting through advertising or employee referrals: Costs, yields, and the effects of geographic focus. European Journal of Work and Organizational Psychology, 14(4), 355-366.

Rosen, P., \& Kluemper, D. H. (2008). The impact of the big five personality traits on the acceptance of social networking website. AMCIS 2008 Proceedings, 274.

Row, H. (2005). "The Next Decade of HR: Trends, Technologies, and Recommendations, In Greutal and Stone (Eds.)", The Brave New World of HER, San Francisco, CA: Jossey-Bass.pp: 255-292.

Ryan, A. M., \& Schmidt, M. J. (1996). An assessment of organizational climate and P-E fit: A tool for organizational change. The International Journal of Organizational Analysis, 4(1), 75-95.

Rynes, S. L., \& Miller, H. E. (1983). Recruiter and job influences on candidates for employment. Journal of Applied Psychology, 68, 147-154.

Rynes, S. L., \& Miller, H. E. (1983). Recruiter and job influences on candidates for employment. Journal of Applied Psychology, 68(1), 147.

Rynes, S. L., Bretz Jr, R. D., \& Gerhart, B. (1991). The importance of recruitment in job choice: A different way of looking. Personnel Psychology, 44(3), 487-521.

Rynes, S., \& Cable, D. (2003). Recruitment. In W. Borman, D. Ilgen, \& R. Klimoski (Eds.), The complete handbook of psychology, (Vol. 12, pp. 55-76). New York: Wiley.

Rynes, S.L. (1991). Recruitment, job choice, and post-hire consequences: A call for new research direction. In M. D. Dunnette \& L.M. Hough (Eds.), Handbook of Industrial and organizational psychology (2nd ed., Vol. 2, pp. 399-444). Palo Alto, CA: Consulting Psychologists Press.

Schein, E. H. (1990). Organizational culture. American Psychologist. Special Issue: Organizational Psychology, 45, 109-119.

Schneider, B. (1987). The people make the place. Personnel Psychology, 40, 437-54.

Schneider, B., Goldstein, \& Smith (2006). The ASA framework: An update. Personnel Psychology, 48, 747-773. 
Schneider, L. (2003) What is branding and how is it important to your marketing strategy? Retrieved from

http://marketing.about.com/cs/brandmktg/a/whatisbranding.htm.

Schreurs, B., Druart, C., Proost, K., \& De Witte, K. (2009). Symbolic attributes and organizational attractiveness: The moderating effects of applicant personality. International Journal of Selection and Assessment, 17(1), 35-46.

Scott, S.G. \& Lane, V.R. (2000). A Stakeholder approach to organizational identity. Academy of Management Review, 25, 43-62.

Shamir, B. (1991). Meaning, self and motivation in organizations. Organization Studies, 12(3), 405-424.

Shih, C. C. W. (2009). The Facebook era: Tapping online social networks to build better products, reach new audiences, and sell more stuff. Prentice-Hall PTR.

Siguaw, J.A., Mattila, A., \& Austin, J. R. (1999). The Brand-Personality Scale. Cornell Hotel and Restaurant Administration Quarterly, 40, 48-55.

Sirgy, M. J. (1985). Using self-congruity and ideal congruity to predict purchase motivation. Journal of Business Research, 13(3), 195-206.

Sirgy, M. J., Grewal, D., Mangleburg, T. F., Park, J. O., Chon, K. S., Claiborne, C. B., ... \& Berkman, H. (1997). Assessing the predictive validity of two methods of measuring self-image congruence. Journal of the academy of marketing science, 25(3), 229-241.

Slaughter, J. E., \& Greguras, G. J. (2009). Initial attraction to organizations: The influence of trait inferences. International Journal of Selection and Assessment, 17(1), 1-18.

Slaughter, J.E., Zickar, M.J., Highhouse, S., \& Mohr, D.C. (2004). Personality trait inferences about organizations: Development of a measure and assessment of construct validity. Journal of Applied Psychology, 89, 85-103.

Spence, M. (1973). Job market signaling. Quarterly Journal of Economics, 87, 355-374.

Spence, M. A. (1974). Market signaling: Information transfer in hiring and related screening processes. Cambridge, MA: Harvard University Press.

Steele, C. M. (1988). The psychology of self-affirmation: Sustaining the integrity of the self. Advances in experimental social psychology, 21, 261-302.

Stewart, D. W., \& Pavlou, P. A. (2002). From consumer response to active consumer: measuring the effectiveness of interactive media. Journal of the Academy of Marketing Science, 30(4), 376-396. 
Stone, D.L., Lukaszewski, K.M. and Isenhour, L. (2005), "e-Recruiting: online strategies for attracting talent", in Gueutal, H.G. and Stone, D.L. (Eds), The Brave New World of eHR, Jossey-Bass, San Francisco, CA, pp. 22-53.

Sumner, C., Byers, A., \& Shearing, M. (2011). Determining personality traits \& privacy concerns from Facebook activity. Black Hat Briefings.

Tajfel, H. and Turner, J.C. (1985), "The social identity theory of group behavior", in Worchel, S. and Austin, W.G. (Eds), Psychology of Intergroup Relations, Vol. 2, Nelson-Hall, Chicago, IL, pp. 7-24.

Thoms, P., Chinn, S. J., Goodrich, J., \& Howard, G. (2004). Designing personable and informative job recruiting web sites: Testing the effect of the design on attractiveness and intent to apply. Psychological reports, 94, 1031-1042.

Thompson, B. 2000. Ten commandments of structural equation modeling. In L.G. Grimm \& P.R. Yarnold (Eds.), Reading and understanding more multivariate statistics: 261-283. Washington, DC: American Psychological Association.

Treadway, C., \& Smith, M. (2010). Facebook marketing: An hour a day. Indianapolis, IN: Wiley.

Turban, D. B. (2001). Organizational attractiveness as an employer on college campuses: An examination of the applicant population. Journal of Vocational Behavior, 58, $293-312$.

Turban, D. B., \& Keon, T. L. (1993). Organizational attractiveness: An interactionist perspective. Journal of Applied Psychology, 78, 184-193.

Turban, D. B., Forret, M. L., \& Hendrickson, C. L. (1998). Applicant attraction to firms: Influences of organization reputation, job and organizational attributes, and recruiter behaviors. Journal of Vocational Behavior, 52(1), 24-44.

Uggerslev, K., Fassina, N., \& Kraichy, D. (2012). Recruiting through the stages: A metaanalytic test of predictors of applicant attraction at different stages of the recruiting process. Personnel Psychology, 65(3), 597-660.

Van Riel, C. B., \& Balmer, J. M. (1997). Corporate identity: the concept, its measurement and management. European Journal of Marketing, 31(5/6), 340355.

Vancouver, J. B., \& Schmitt, N. W. (1991). An exploratory examination of person-organization fit: Organizational goal congruence. Personnel psychology, 44(2), 333-352.

Watson, D. (1989). Strangers' ratings of the five robust personality factors: Evidence of a surprising convergence with self-report. Journal of Personality and Social Psychology, 57(1), 120. 
Whetten, D. A., Lewis, D., \& Mischel, L. J. (1992). Towards an integrated model of organizational identity and member commitment. Paper presented at the annual meeting of Academy of Managememnt, Las Vegas.

Williamson, I. O., King Jr, J. E., Lepak, D., \& Sarma, A. (2010). Firm reputation, recruitment web sites, and attracting applicants. Human Resource Management, 49(4), 669-687.

Williamson, I.O., Lepak, D. P., \& King, J. (2003). The effect of company recruitment web site orientation on individuals' perceptions of organizational attractiveness. Journal of Vocational Behavior, 63, 242-263.

Witt, L. A., \& Nye, L. G. (1992). Gender and the relationship between perceived fairness of pay or promotion and job satisfaction. Journal of Applied Psychology, 77(6), 910.

Zusman, R. R., \& Landis, R. S. (2002). Applicant preferences for web-based versus traditional job postings. Computers in Human Behavior, 18, 285-296.

Zusman, R. R., \& Landis, R. S. (2002). Applicant preferences for web-based versus traditional job postings. Computers in Human Behavior, 18(3), 285-296. 
Appendix A. Summary of Research Questions 1-5: Objective Indicators of Image

\begin{tabular}{|c|c|c|c|}
\hline $\begin{array}{c}\text { Dimensi } \\
\text { on }\end{array}$ & Definition & Website & Facebook \\
\hline \multirow[t]{4}{*}{$\begin{array}{c}\text { Boy } \\
\text { Scout }\end{array}$} & $\begin{array}{l}\text { - friendly } \\
\text { - pleasant }\end{array}$ & $\begin{array}{l}\text { - } \text { site visitors } \\
\text { - awards for best places to } \\
\text { work } \\
\text { - number of photos of } \\
\text { people }\end{array}$ & $\begin{array}{l}\text { - page likes } \\
\text { - awards for best places to work } \\
\text { - number of photos of people } \\
\text { - frequency of posts }\end{array}$ \\
\hline & $\begin{array}{l}\text { - family- } \\
\text { oriented } \\
\text { - attentive } \\
\text { to people } \\
\text { - personal }\end{array}$ & $\begin{array}{l}\text { - employee recognition } \\
\text { - frequency word 'support' } \\
\text { - discussion forum } \\
\text { - frequency of word } \\
\text { 'diversity' }\end{array}$ & $\begin{array}{l}\text { - fan recognition } \\
\text { - frequency word 'support' posted } \\
\text { - interactive posts } \\
\text { - frequency of word 'diversity' }\end{array}$ \\
\hline & $\begin{array}{l}\text { - cooperativ } \\
\text { e } \\
\text { - helpful }\end{array}$ & $\begin{array}{l}\text { - contact information } \\
\text { - benefits listed } \\
\text { - continuing education } \\
\text { information }\end{array}$ & $\begin{array}{l}\text { - contact information } \\
\text { - benefits listed } \\
\text { - continuing education information }\end{array}$ \\
\hline & $\begin{array}{l}\text { - honest } \\
\text { - clean }\end{array}$ & $\begin{array}{l}\text { - environmental-awareness } \\
\text { - community involvement } \\
\text { - frequency of word 'trust' } \\
\text { - frequency of word } \\
\text { 'respect' }\end{array}$ & $\begin{array}{l}\text { - environmental-awareness posts } \\
\text { - community involvement posts } \\
\text { - frequency of word 'trust' } \\
\text { - frequency of word 'respect' }\end{array}$ \\
\hline \multirow[t]{2}{*}{$\begin{array}{c}\text { Innovat } \\
\text { ion }\end{array}$} & $\begin{array}{l}\text { - interesting } \\
\text { exciting } \\
\text { - not boring }\end{array}$ & $\begin{array}{l}\text { - } \text { Training/education } \\
\text { opportunities } \\
\text { - advertising new } \\
\text { product/service launches } \\
\text { - frequency of word } \\
\text { 'innovation' } \\
\text { - frequency of word } \\
\text { 'technology' } \\
\text { - frequency of word 'risk' }\end{array}$ & $\begin{array}{l}\text { - Training/education opportunities } \\
\text { - advertising new product/service } \\
\text { launches } \\
\text { - frequency of word 'innovation' } \\
\text { - frequency of word 'technology' } \\
\text { - frequency of word 'risk' }\end{array}$ \\
\hline & $\begin{array}{l}\text { - unique } \\
\text { - original } \\
\text { - not plain }\end{array}$ & $\begin{array}{l}\text { - links to other social media } \\
\text { - use of color in text } \\
\text { - language choice in main } \\
\text { page } \\
\text { - use of flash or video } \\
\text { - links within careers } \\
\text { section } \\
\text { - about us length }\end{array}$ & $\begin{array}{l}\text { - links to other social media } \\
\text { - use of color in text } \\
\text { - language choice in 'about us' } \\
\text { - video posts } \\
\text { - links within profile } \\
\text { - about us length }\end{array}$ \\
\hline
\end{tabular}




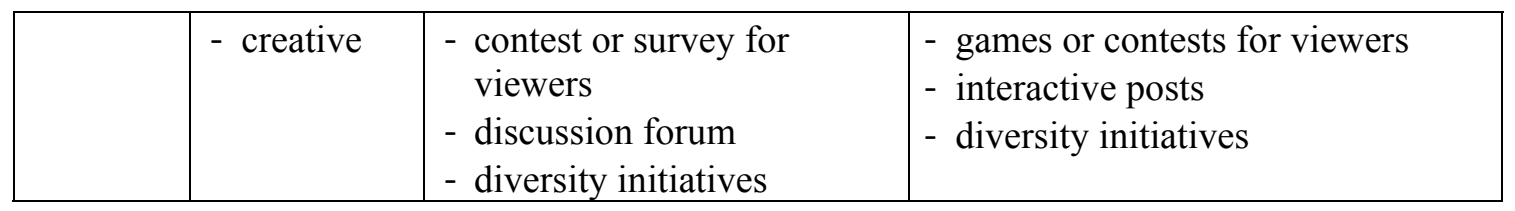




\begin{tabular}{|c|c|c|c|}
\hline Dimension & Definition & Website & Facebook \\
\hline \multirow[t]{3}{*}{ Dominance } & - successful & $\begin{array}{l}\text { - performance awards listed } \\
\text { - financial information } \\
\text { provided } \\
\text { - use of flow chart/diagram } \\
\text { - bonus-system listed } \\
\text { - frequency of word } \\
\text { 'success' }\end{array}$ & $\begin{array}{l}\text { - performance awards listed } \\
\text { - financial information } \\
\text { provided } \\
\text { - use of flow chart/diagram } \\
\text { - bonus-system listed } \\
\text { - frequency of word 'success' }\end{array}$ \\
\hline & $\begin{array}{l}\text { - dominant } \\
\text { - popular }\end{array}$ & $\begin{array}{l}\text { - media articles listed } \\
\text { - links within careers } \\
\text { section } \\
\text { - language availability of } \\
\text { webpage } \\
\text { - site traffic counter } \\
\text { - frequency of word } \\
\text { 'winning' }\end{array}$ & $\begin{array}{l}\text { - media articles posted } \\
\text { - links within profile } \\
\text { - page likes } \\
\text { - people talking about page } \\
\text { - frequency of word } \\
\text { 'winning' }\end{array}$ \\
\hline & $\begin{array}{l}\text { - busy } \\
\text { active }\end{array}$ & $\begin{array}{l}\text { - community involvement } \\
\text { - events listed } \\
\text { - charity information }\end{array}$ & $\begin{array}{l}\text { - community involvement } \\
\text { posts } \\
\text { - events listed } \\
\text { - charity information } \\
\text { - frequency of posts }\end{array}$ \\
\hline \multirow[t]{3}{*}{ Thrift } & $\begin{array}{l}\text { - low } \\
\text { budget } \\
\text { - poor } \\
\text { - low class } \\
\text { - deprived }\end{array}$ & $\begin{array}{l}\text { - amount images/ photos* } \\
\text { - use of flash or video* } \\
\text { - frequency of word } \\
\text { 'budget' }\end{array}$ & $\begin{array}{l}\text { - amount images/ photos* } \\
\text { - video posts* } \\
\text { - frequency of word 'budget' }\end{array}$ \\
\hline & $\begin{array}{ll}\text { - } & \text { simple } \\
\text { - } & \text { reduced } \\
& \text { undersized }\end{array}$ & $\begin{array}{l}\text { - amount of text* } \\
\text { - links within careers } \\
\text { section* } \\
\text { - links to other social } \\
\text { media* }\end{array}$ & $\begin{array}{l}\text { - amount of text in 'about } \\
\text { us'* } \\
\text { - links within profile* } \\
\text { - links to other social media* } \\
\text { - frequency of posts* }\end{array}$ \\
\hline & - sloppy & $\begin{array}{l}\text { - spelling mistakes } \\
\text { - frequency of word } \\
\text { 'safety* }\end{array}$ & $\begin{array}{l}\text { - spelling mistakes } \\
\text { - frequency of word 'safety'* }\end{array}$ \\
\hline Style & $\begin{array}{l}\text { - stylish } \\
\text { - fashionabl } \\
\text { e }\end{array}$ & $\begin{array}{l}\text { - number of photos } \\
\text { - amount of graphics/art } \\
\text { - use of flash or video } \\
\text { - music on site } \\
\text { - use of color in text }\end{array}$ & $\begin{array}{l}\text { - number of photos } \\
\text { - amount of graphics/art } \\
\text { - video posts } \\
\text { - music/audio posts } \\
\text { - use of color in text }\end{array}$ \\
\hline
\end{tabular}




\begin{tabular}{|c|c|c|}
\hline $\begin{array}{l}\text { - hip } \\
\text { - trendy }\end{array}$ & $\begin{array}{l}\text { - links to other social media } \\
\text { - events listed } \\
\text { - celebrity or athlete } \\
\text { endorsements }\end{array}$ & $\begin{array}{l}\text { - links to other social media } \\
\text { - events listed } \\
\text { - celebrity or athlete posts }\end{array}$ \\
\hline - creative & $\begin{array}{l}\text { - contest or survey for } \\
\text { viewers } \\
\text { - discussion forum } \\
\text { - diversity initiatives }\end{array}$ & $\begin{array}{l}\text { - games or contests for } \\
\text { viewers } \\
\text { - interactive posts } \\
\text { - diversity initiatives }\end{array}$ \\
\hline
\end{tabular}

* indicates low levels or absence of that indicator 
Appendix B. List of Companies Included in the Study $(N=102)$

\begin{tabular}{|c|c|c|}
\hline ID & Rank & Company \\
\hline 1 & 500 & $\begin{array}{l}\text { Molina } \\
\text { Healthcare }\end{array}$ \\
\hline 2 & 497 & Erie Insurance \\
\hline 3 & 496 & Rockwell Collins \\
\hline 4 & 495 & Smuckers \\
\hline 5 & 491 & $\begin{array}{l}\text { Alliant } \\
\text { Techsystems } \\
\text { (ATK) }\end{array}$ \\
\hline 6 & 490 & MetroPCS \\
\hline 7 & 489 & CIT Group \\
\hline 8 & 485 & Charles Schwab \\
\hline 9 & 483 & Yahoo \\
\hline 10 & 482 & $\begin{array}{l}\text { Western \& } \\
\text { Southern } \\
\text { Financial Group }\end{array}$ \\
\hline 11 & 481 & Meritor \\
\hline 12 & 480 & $\begin{array}{l}\text { SunGard Data } \\
\text { Systems }\end{array}$ \\
\hline 13 & 478 & United Stationers \\
\hline 14 & 475 & $\begin{array}{l}\text { FMC } \\
\text { Technologies }\end{array}$ \\
\hline 15 & 474 & NetApp \\
\hline 16 & 473 & $\begin{array}{l}\text { Casey's General } \\
\text { Stores }\end{array}$ \\
\hline 17 & 467 & Big Lots \\
\hline 18 & 466 & $\begin{array}{l}\text { Dick's Sporting } \\
\text { Goods }\end{array}$ \\
\hline 19 & 465 & Gannett \\
\hline 20 & 464 & $\begin{array}{l}\text { Frontier } \\
\text { Communications }\end{array}$ \\
\hline 21 & 461 & Pitney Bowes \\
\hline 22 & 460 & $\begin{array}{l}\text { Insight } \\
\text { Enterprises }\end{array}$ \\
\hline 23 & 459 & Con-way Freight \\
\hline 24 & 458 & Harley-Davidson \\
\hline 25 & 456 & Clorox \\
\hline 26 & 454 & Owens Corning \\
\hline 27 & 450 & $\begin{array}{l}\text { Live Nation } \\
\text { Entertainment }\end{array}$ \\
\hline 28 & 447 & NCR \\
\hline 29 & 445 & Western Union \\
\hline 30 & 444 & $\begin{array}{l}\text { Kindred } \\
\text { Healthcare }\end{array}$ \\
\hline 31 & 442 & Avaya \\
\hline 32 & 441 & Kelly Services \\
\hline 33 & 440 & CH2M Hill \\
\hline
\end{tabular}

\begin{tabular}{|c|c|c|}
\hline ID & Rank & Company \\
\hline 34 & 439 & $\begin{array}{l}\text { Booz Allen } \\
\text { Hamilton }\end{array}$ \\
\hline 35 & 438 & Spectra Energy \\
\hline 36 & 436 & Domtar \\
\hline 37 & 435 & Foot Locker \\
\hline 38 & 434 & $\begin{array}{l}\text { Starwood Hotels } \\
\& \text { Resorts }\end{array}$ \\
\hline 39 & 431 & Ralph Lauren \\
\hline 40 & 430 & SanDisk \\
\hline 41 & 429 & $\begin{array}{l}\text { Auto-Owners } \\
\text { Insurance }\end{array}$ \\
\hline 42 & 428 & Emcor Group \\
\hline 43 & 424 & $\begin{array}{l}\text { O'Reilly } \\
\text { Automotive }\end{array}$ \\
\hline 44 & 422 & Exelis \\
\hline 45 & 420 & Pacific Life \\
\hline 46 & 417 & $\begin{array}{l}\text { Dr Pepper } \\
\text { Snapple Group }\end{array}$ \\
\hline 47 & 414 & Rubbermaid \\
\hline 48 & 410 & $\begin{array}{l}\text { Rockwell } \\
\text { Automation }\end{array}$ \\
\hline 49 & 408 & Kodak \\
\hline 50 & 407 & Ryder \\
\hline 51 & 404 & Hershey's \\
\hline 52 & 400 & PetSmart \\
\hline 53 & 399 & Consol Energy \\
\hline 54 & 397 & Wesco \\
\hline 55 & 394 & $\begin{array}{l}\text { International } \\
\text { iHeartRADIO }\end{array}$ \\
\hline 56 & 392 & $\begin{array}{l}\text { Advance Auto } \\
\text { Parts }\end{array}$ \\
\hline 57 & 391 & Symantec \\
\hline 58 & 388 & Mattel \\
\hline 59 & 387 & $\begin{array}{l}\text { Precision } \\
\text { Castparts }\end{array}$ \\
\hline 60 & 385 & Amerigroup \\
\hline 61 & 383 & Dillard's \\
\hline 62 & 382 & $\begin{array}{l}\text { American Family } \\
\text { Insurance }\end{array}$ \\
\hline 63 & 379 & Terex \\
\hline 64 & 378 & $\begin{array}{l}\text { Advanced Micro } \\
\text { Devices (AMD) }\end{array}$ \\
\hline 65 & 376 & $\begin{array}{l}\text { Sanmina } \\
\text { Corporation }\end{array}$ \\
\hline 66 & 375 & $\begin{array}{l}\text { Agilent } \\
\text { Technologies }\end{array}$ \\
\hline 67 & 373 & Dollar Tree \\
\hline
\end{tabular}




\begin{tabular}{lll}
\hline ID & Rank & Company \\
\hline 68 & 372 & Fifth Third \\
& & Bancorp \\
69 & 370 & MasterCard \\
70 & 368 & Celanese \\
71 & 367 & Avery Dennison \\
72 & 366 & Cliffs Natural \\
& & Resources \\
73 & 365 & Ecolab \\
74 & 363 & Winn-Dixie \\
75 & 360 & Barnes \& Noble \\
76 & 358 & Interpublic Group \\
& & (IPG) \\
77 & 356 & Alpha Natural \\
& & Resources \\
78 & 354 & OfficeMax \\
79 & 351 & Charter \\
& & Communications \\
80 & 349 & Dole \\
81 & 346 & Eastman \\
& & Chemical \\
82 & 343 & Company \\
83 & 341 & Regions Bank \\
& & Diagt \\
84 & 340 & Ameren \\
85 & 337 & Oshkosh B'gosh \\
86 & 335 & Boston Scientific
\end{tabular}

\begin{tabular}{ccl}
\hline ID & Rank & Company \\
\hline 87 & 334 & Campbell Soup \\
88 & 332 & Thrivent \\
& & Financial for \\
& & Lutherans \\
89 & 331 & MGM Resorts \\
& & International \\
90 & 330 & Sonic \\
& & Automotive \\
91 & 322 & AECOM \\
& & Technology \\
92 & 320 & AutoZone \\
93 & 318 & Grainger \\
94 & 309 & Hertz \\
95 & 301 & Family Dollar \\
96 & 300 & Discover \\
97 & 299 & Ross Stores \\
98 & 295 & Principal \\
& & Financial \\
99 & 294 & Bed Bath \& \\
& & Beyond \\
100 & 293 & Sherwin- \\
& & Williams \\
101 & 292 & AGCO \\
102 & 290 & Estee Lauder \\
\hline
\end{tabular}




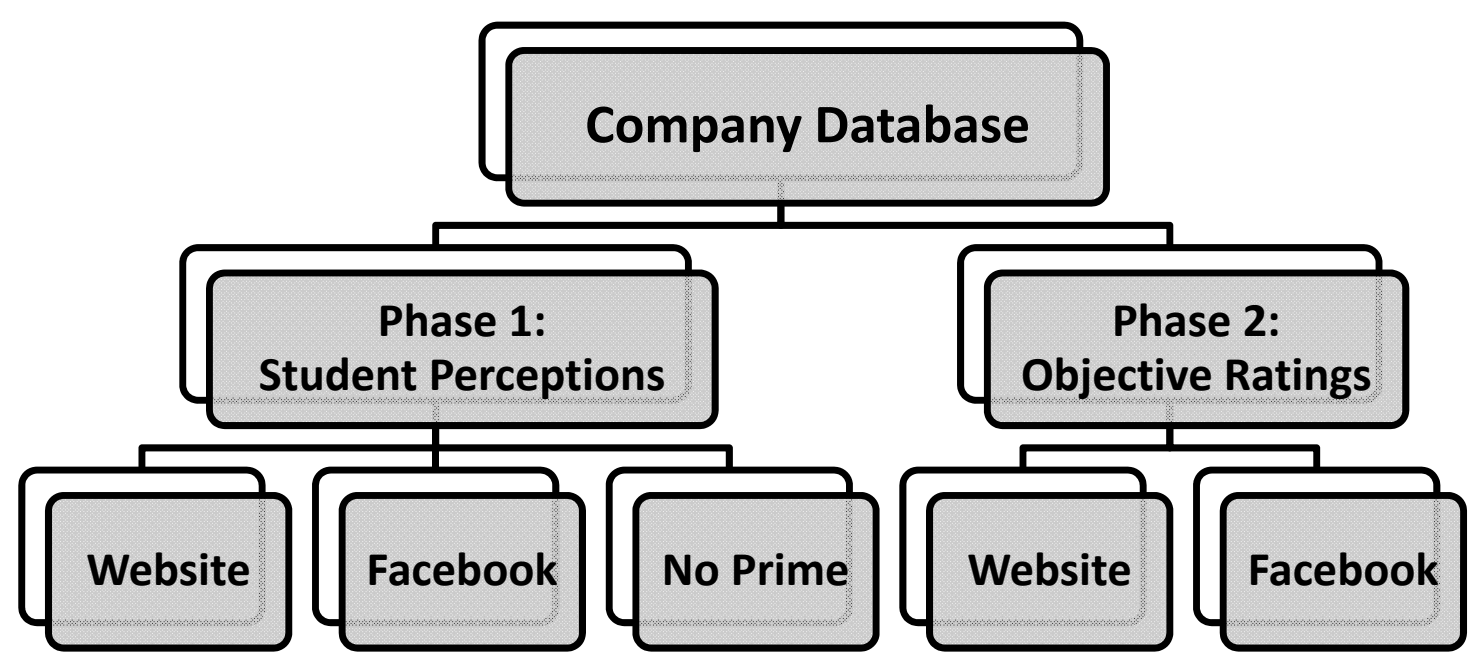




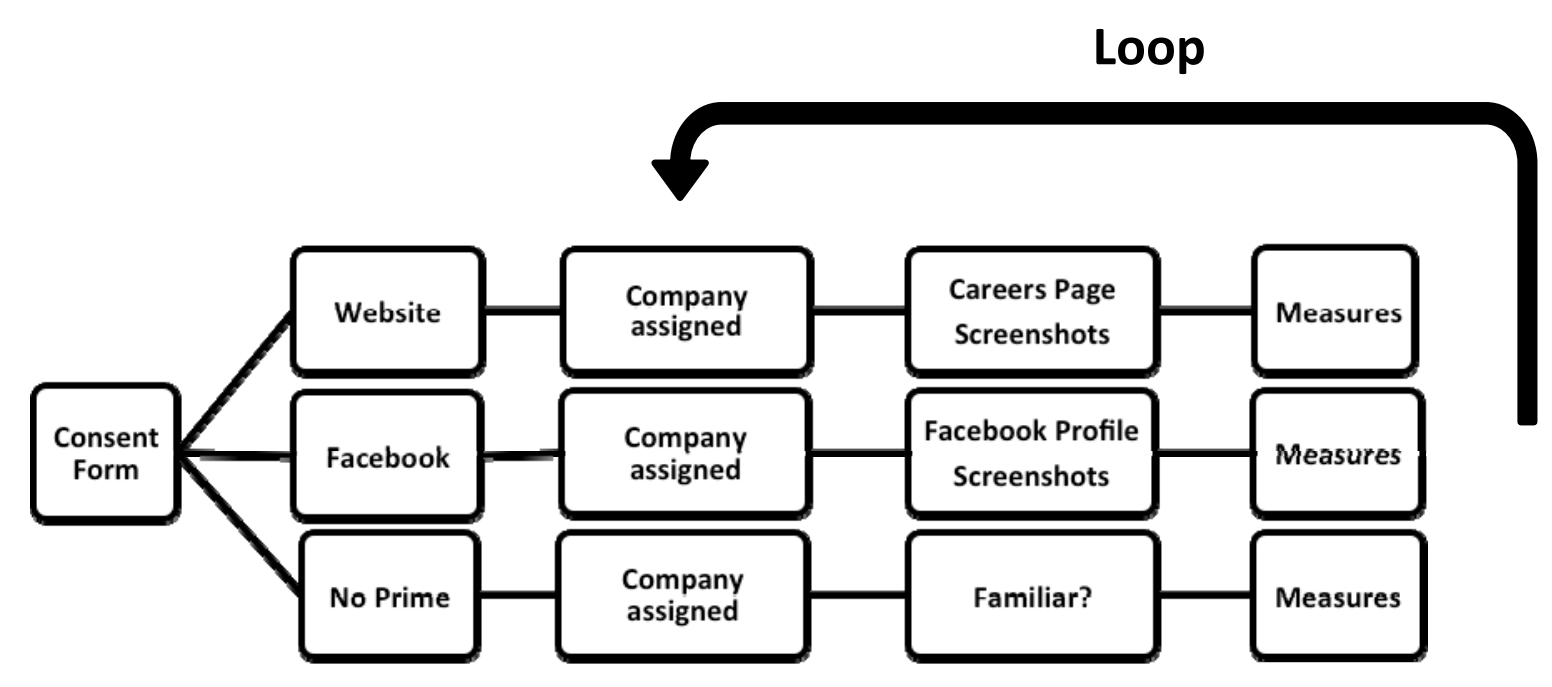


Appendix E. List of Companies Retained for Phase One No Exposure Condition $(N=50)$

\begin{tabular}{|c|c|}
\hline Rank & Company Name for Survey \\
\hline 495 & Smuckers \\
\hline 490 & MetroPCS \\
\hline 485 & Charles Schwab \\
\hline 483 & Yahoo \\
\hline 474 & NetApp \\
\hline 467 & Big Lots \\
\hline 466 & Dick's Sporting Goods \\
\hline 458 & Harley-Davidson \\
\hline 456 & Clorox \\
\hline 445 & Western Union \\
\hline 435 & Foot Locker \\
\hline 434 & Starwood Hotels \& Resorts \\
\hline 431 & Ralph Lauren \\
\hline 430 & SanDisk \\
\hline 424 & O'Reilly Automotive \\
\hline 417 & Dr Pepper Snapple Group \\
\hline 414 & Rubbermaid \\
\hline 408 & Kodak \\
\hline 407 & Ryder \\
\hline 404 & Hershey's \\
\hline 400 & PetSmart \\
\hline 399 & Consol Energy \\
\hline 394 & iHeartRADIO \\
\hline 392 & Advance Auto Parts \\
\hline 391 & Symantec \\
\hline 388 & Mattel \\
\hline 385 & Amerigroup \\
\hline 383 & Dillard's \\
\hline 373 & Dollar Tree \\
\hline 370 & MasterCard \\
\hline 363 & Winn-Dixie \\
\hline 360 & Barnes \& Noble \\
\hline 358 & Interpublic Group (IPG) \\
\hline 354 & OfficeMax \\
\hline 349 & Dole \\
\hline 343 & Regions Bank \\
\hline 341 & Quest Diagnostics \\
\hline 337 & Oshkosh B'gosh \\
\hline 335 & Boston Scientific \\
\hline 334 & Campbell Soup \\
\hline 331 & MGM Resorts International \\
\hline 330 & Sonic Automotive \\
\hline 320 & AutoZone \\
\hline 309 & Hertz \\
\hline 301 & Family Dollar \\
\hline 300 & Discover \\
\hline
\end{tabular}

\begin{tabular}{ll}
\hline Rank & Company Name for Survey \\
\hline 299 & Ross Stores \\
294 & Bed Bath \& Beyond \\
293 & Sherwin-Williams \\
290 & Estee Lauder \\
\hline
\end{tabular}


Appendix F. Perceptions of Organizational Personality Measure

Participant instructions:

Please describe the extent to which the following adjectives describe the organization presented using the following scale

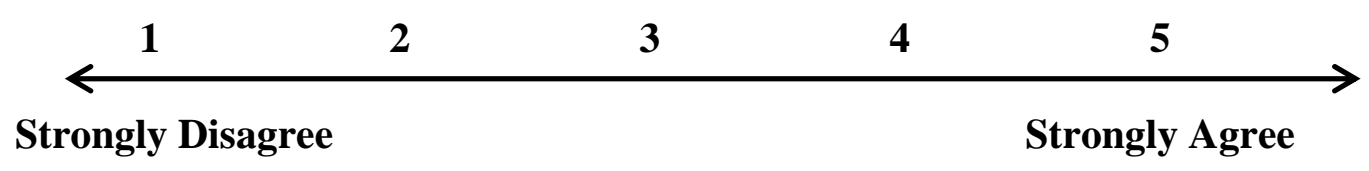

Boy Scout

1. Friendly
3. Pleasant
5. Cooperative
7. Helpful
9. Honest

2. Attentive to People
4. Family-oriented
6. Personal
8. Clean

Innovation

10. Interesting
12. Unique
14. Boring*
16. Original

11. Exciting
13. Creative
15. Plain*

Dominance

17. Successful

19. Dominant

21. Active

18. Popular

20. Busy

Thriftiness

22. Low budget

23. Low class

24. Simple

25. Reduced

26. Sloppy

27. Poor

28. Undersized

29. Deprived

Stylishness

30. Stylish

31. Fashionable

32. Hip

33. Trendy

*Indicates reverse scored item.

(Slaughter, Zickar, Highhouse, \& Mohr, 2004) 


\section{ORGANIZATIONAL PERSONALITY: Website SME SORT TASK}

You have been assigned to the website SME group. You will be provided with a list of website components/features that have been theoretically and empirically linked to organizational personality dimensions. You will be asked to identify which (if any) organizational personality dimension each item represents. Your help with this task will be used as part of the content validation process.

The following section provides a brief overview of the study as well as detailed definition of each organizational personality dimension. A summary of the definition will also be provided within the online sort task.

\section{Study Background}

A key way organizations can differentiate themselves is through their organizational image. Slaughter, Zickar, Highhous, and Mohr (2004) developed the construct of organizational personality, defined as the "set of human personality characteristics perceived to be associated with an organization (p.86)." This five dimensional construct consists of five personality dimensions: Boy Scout, Innovation, Dominance, Thrift, and Style.

Organization personality is shaped by the different ways the organization presents itself to the public. Examples of these possible channels for organization personality projections include television/radio advertisements, media coverage, the Internet, and personal familiarity with the organization (Slaughter et al., 2004).

Researchers assert that outsiders are able to make an assessment of an organization's personality even when dealing with very limited exposure to the organization (Lievens \& Highhouse, 2003; Slaughter et al., 2004). Additionally, signaling theory suggests that in the face of incomplete information about an organization, individuals will call on whatever information is available to make inferences about unknown organizational attributes (Rynes, 1991; Spence, 1973).

Consequently, a central goal of this research is to identify aspects of web-based recruitment media that influence viewer perceptions organizational personality.

\section{Organizational Personality Dimensions}

Boy Scout: Refers to an organization's honesty, helpfulness, attentiveness, friendliness and family-orientation. Organizations perceived to be strong on this dimension are Target, Disney, and Johnson \& Johnson.

Innovation: Relates to how unique, interesting, or creative an organization is viewed. Organizations perceived as highly innovative include Apple, PepsiCo, and Microsoft. 
Dominance: Corresponds to an organization being associated with success, popularity, or high-activity levels. Organizations perceived to be strong on the Dominance dimension include Coca-Cola, General Motors, Disney, and AT\&T.

Thrift: Describes organizations that are seen as low budget, small, or sloppy. Organizations perceived as being strong in this dimension include K-Mart, Kroger, Wal-Mart, Subway, and J. C. Penney.

Style: $\quad$ Represents perceptions of hipness, being contemporary, or trendy. Organizations perceived as rating highly on this dimension include Nike, Pepsi, and T-Mobile are rated highly on this dimension 
SME Instructions:

Please take a moment to familiarize yourself with the definitions of each organizational personality dimension which have been provided to you. Please feel free to ask for clarification on any of the dimensions.

When you are ready, please indicate the dimension(s) you feel each indicator represents. You may select as many dimensions as you would like. If you feel that an indicator does not represent any of the dimensions, please select the last column marked 'N/A' for that row.

\begin{tabular}{|l|l|l|l|l|l|l|}
\hline \multirow{2}{*}{$\begin{array}{l}\text { Website } \\
\text { Indicator* }\end{array}$} & \multicolumn{5}{|c|}{ Organizational Personality Dimensions } & \\
\cline { 2 - 7 } & Boy Scout & Innovation & Dominance & Thriftiness & Stylishness & N/A \\
\hline Indicator 1 & & & & & & \\
\hline Indicator 2 & & & & & & \\
\hline Indicator 3 & & & & & & \\
\hline Indicator 4 & & & & & & \\
\hline$\ldots$ & & & & & & \\
\hline$\ldots$ & & & & & & \\
\hline
\end{tabular}

* This task was repeated for Facebook indicators 


\begin{tabular}{|c|c|c|c|c|c|c|}
\hline & Website Indicator & 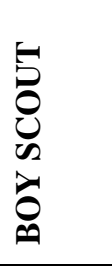 & 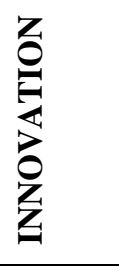 & 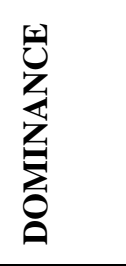 & 舀 & 攻 \\
\hline \multirow{29}{*}{ 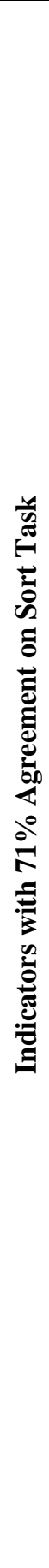 } & \multirow{2}{*}{$\begin{array}{l}\text { Awards for best places to work listed } \\
\text { Contact information listed }\end{array}$} & 4 & 3 & 5 & 1 & 2 \\
\hline & & 5 & $\mathbf{0}$ & 1 & 1 & $\mathbf{0}$ \\
\hline & Count of total website visitors & 2 & $\mathbf{0}$ & 5 & $\mathbf{0}$ & 2 \\
\hline & Financial information listed & 3 & $\mathbf{0}$ & 5 & $\mathbf{0}$ & $\mathbf{0}$ \\
\hline & Frequency of word 'budget' & 1 & $\mathbf{0}$ & $\mathbf{0}$ & 6 & $\mathbf{0}$ \\
\hline & Frequency of word 'innovation' & $\mathbf{0}$ & 7 & 2 & $\mathbf{0}$ & 2 \\
\hline & Frequency of word 'respect' & 7 & $\mathbf{0}$ & 3 & 1 & $\mathbf{0}$ \\
\hline & Frequency of word 'risk' & $\mathbf{0}$ & 5 & 3 & 1 & $\mathbf{0}$ \\
\hline & Frequency of word 'safety' & 7 & $\mathbf{0}$ & $\mathbf{0}$ & 1 & $\mathbf{0}$ \\
\hline & Frequency of word 'success' & $\mathbf{0}$ & 1 & 7 & $\mathbf{0}$ & $\mathbf{0}$ \\
\hline & Frequency of word 'technology' & $\mathbf{0}$ & 7 & $\mathbf{0}$ & $\mathbf{0}$ & 3 \\
\hline & Frequency of word 'trust' & 7 & $\mathbf{0}$ & $\mathbf{0}$ & 1 & $\mathbf{0}$ \\
\hline & Frequency of word 'winning' & $\mathbf{0}$ & $\mathbf{0}$ & 7 & $\mathbf{0}$ & $\mathbf{0}$ \\
\hline & \multirow{2}{*}{$\begin{array}{l}\text { Frequency word 'support' } \\
\text { Information about bonus-system listed }\end{array}$} & 7 & 1 & $\mathbf{0}$ & $\mathbf{0}$ & 1 \\
\hline & & 2 & 3 & 5 & $\mathbf{0}$ & 1 \\
\hline & \multirow{2}{*}{$\begin{array}{l}\text { Information about charity work listed } \\
\text { Information about community } \\
\text { involvement listed }\end{array}$} & 6 & 2 & $\mathbf{0}$ & $\mathbf{0}$ & 3 \\
\hline & & 5 & 2 & $\mathbf{0}$ & $\mathbf{0}$ & 4 \\
\hline & $\begin{array}{l}\text { Information about employee benefits } \\
\text { listed }\end{array}$ & 5 & $\mathbf{0}$ & 1 & 1 & $\mathbf{0}$ \\
\hline & \multirow{2}{*}{$\begin{array}{l}\text { Information about environmental- } \\
\text { awareness listed } \\
\text { Information about performance awards } \\
\text { listed }\end{array}$} & 4 & 3 & 1 & $\mathbf{0}$ & 6 \\
\hline & & $\mathbf{0}$ & $\mathbf{0}$ & 7 & 1 & $\mathbf{0}$ \\
\hline & $\begin{array}{l}\text { Length of 'about us' section (\# of words) } \\
\text { Number of flow charts/diagrams/graphs }\end{array}$ & 3 & 1 & 2 & 5 & $\mathbf{1}$ \\
\hline & $\begin{array}{l}\text { Number of flow charts/diagrams/graphs } \\
\text { visible }\end{array}$ & 1 & 3 & 5 & $\mathbf{0}$ & 3 \\
\hline & \multirow{2}{*}{$\begin{array}{l}\text { Number of graphics/artwork } \\
\text { Number of links to other [social] media } \\
\text { sites }\end{array}$} & $\mathbf{0}$ & $\mathbf{1}$ & $\mathbf{0}$ & 1 & 7 \\
\hline & & 1 & 3 & 1 & $\mathbf{0}$ & 6 \\
\hline & Number of spelling mistakes & $\mathbf{0}$ & $\mathbf{0}$ & $\mathbf{0}$ & 7 & 1 \\
\hline & Option of music/audio on site & $\mathbf{0}$ & 3 & $\mathbf{0}$ & $\mathbf{0}$ & 6 \\
\hline & $\begin{array}{l}\text { Option of viewing webpage in a } \\
\text { different language available }\end{array}$ & 4 & 5 & 3 & $\mathbf{0}$ & 4 \\
\hline & Presence of a discussion forum & 5 & 3 & $\mathbf{0}$ & $\mathbf{0}$ & 3 \\
\hline & $\begin{array}{l}\text { Presence of advertisements for new } \\
\text { products/service launches }\end{array}$ & $\mathbf{0}$ & 5 & $\mathbf{0}$ & 2 & 1 \\
\hline
\end{tabular}




\begin{tabular}{|c|c|c|c|c|c|c|}
\hline & Website Indicator & $\begin{array}{l}-1 \\
0 \\
0 \\
0 \\
\omega \\
2 \\
0 \\
0\end{array}$ & 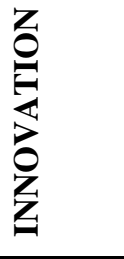 & 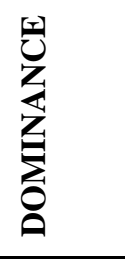 & 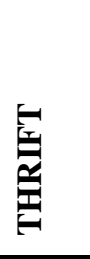 & 点 \\
\hline & $\begin{array}{l}\text { Presence of an employee recognition } \\
\text { program }\end{array}$ & 6 & $\mathbf{0}$ & 1 & 1 & 1 \\
\hline & $\begin{array}{l}\text { Presence of celebrity or athlete } \\
\text { endorsement }\end{array}$ & $\mathbf{0}$ & $\mathbf{0}$ & 3 & 1 & 5 \\
\hline & Total number of images/photos & 1 & 2 & $\mathbf{0}$ & 5 & 5 \\
\hline & Use of flash or video & 1 & 5 & $\mathbf{0}$ & 1 & 5 \\
\hline \multirow{11}{*}{ 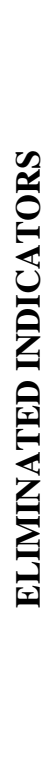 } & \multirow{11}{*}{$\begin{array}{l}\text { Continuing education information listed } \\
\text { Frequency of word 'diversity' } \\
\text { Information about diversity initiatives } \\
\text { listed } \\
\text { Information about training/education } \\
\text { opportunities } \\
\text { Information about upcoming/past events } \\
\text { listed } \\
\text { Number of media articles listed } \\
\text { Number of photos of } \\
\text { employees/customers/applicants } \\
\text { Number of webpage links within the } \\
\text { careers section } \\
\text { Number of website visitors } \\
\text { Presence of contest or survey for page } \\
\text { visitors } \\
\text { Use of color in text }\end{array}$} & 4 & 3 & 1 & $\mathbf{0}$ & $\mathbf{0}$ \\
\hline & & 3 & 4 & $\mathbf{0}$ & $\mathbf{0}$ & 3 \\
\hline & & 4 & 2 & $\mathbf{0}$ & 0 & 4 \\
\hline & & 4 & 3 & 1 & 1 & $\mathbf{0}$ \\
\hline & & 1 & 1 & 2 & $\mathbf{0}$ & 2 \\
\hline & & $\mathbf{0}$ & 1 & 3 & $\mathbf{0}$ & 4 \\
\hline & & 4 & 1 & $\mathbf{0}$ & 1 & 3 \\
\hline & & 2 & 1 & 3 & $\mathbf{0}$ & 1 \\
\hline & & 2 & $\mathbf{0}$ & 3 & 1 & 2 \\
\hline & & 1 & 2 & $\mathbf{0}$ & 1 & 1 \\
\hline & & 1 & 2 & 1 & 2 & 4 \\
\hline
\end{tabular}

Note. Shaded cells represent original classifications based on RQ 1-5. Cell values represent total number of SMEs who chose that OP dimension out of total possible $\mathrm{N}=7$. 


\begin{tabular}{|c|c|c|c|c|c|}
\hline ITEM & 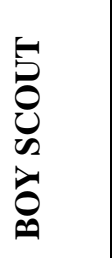 & 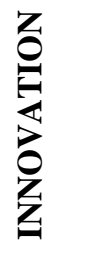 & 恐 & 至 & 究 \\
\hline \multirow{2}{*}{$\begin{array}{l}\text { Awards for best places to work posted } \\
\text { Contact information available }\end{array}$} & 5 & 3 & 5 & $\mathbf{0}$ & 3 \\
\hline & 7 & 1 & $\mathbf{0}$ & 1 & 1 \\
\hline Frequency of word 'budget' & 0 & 1 & 1 & 6 & $\mathbf{0}$ \\
\hline Frequency of word 'diversity' & 5 & 4 & 1 & 1 & 6 \\
\hline Frequency of word 'innovation' & 0 & 7 & 1 & $\mathbf{0}$ & 3 \\
\hline Frequency of word 'respect' & 6 & $\mathbf{0}$ & 2 & $\mathbf{0}$ & $\mathbf{0}$ \\
\hline Frequency of word 'risk' & $\mathbf{0}$ & 5 & 2 & 1 & 4 \\
\hline Frequency of word 'safety' & 6 & $\mathbf{0}$ & $\mathbf{0}$ & $\mathbf{0}$ & $\mathbf{0}$ \\
\hline Frequency of word 'success' & 1 & 5 & 7 & 2 & 1 \\
\hline Frequency of word 'support' & 7 & 1 & $\mathbf{0}$ & 1 & $\mathbf{0}$ \\
\hline Frequency of word 'technology' & $\mathbf{0}$ & 7 & 2 & $\mathbf{0}$ & 4 \\
\hline Frequency of word 'trust' & 7 & $\mathbf{0}$ & 1 & 1 & $\mathbf{0}$ \\
\hline Frequency of word 'winning' & $\mathbf{0}$ & 4 & 7 & $\mathbf{0}$ & 1 \\
\hline Information about bonus-system posted & 4 & 2 & $\mathbf{0}$ & 5 & 1 \\
\hline Information about charity work posted & 7 & $\mathbf{0}$ & 1 & 2 & 2 \\
\hline \multirow{2}{*}{$\begin{array}{l}\text { Information about community } \\
\text { involvement posted } \\
\text { Information about continuing education } \\
\text { posted }\end{array}$} & 7 & 0 & 0 & 0 & 1 \\
\hline & 5 & 5 & 2 & $\mathbf{0}$ & 1 \\
\hline \multirow{2}{*}{$\begin{array}{l}\text { Information about employee benefits } \\
\text { posted } \\
\text { Information about training/education } \\
\text { opportunities posted }\end{array}$} & 6 & 2 & 3 & 0 & 1 \\
\hline & 4 & 6 & 4 & $\mathbf{0}$ & 1 \\
\hline \multirow{2}{*}{$\begin{array}{l}\text { Length of 'about' section } \\
\text { Number of celebrity or athlete -related } \\
\text { posts }\end{array}$} & 5 & 2 & 4 & 5 & 1 \\
\hline & 1 & 2 & 3 & 2 & 6 \\
\hline $\begin{array}{l}\text { Number of links to other [social] media } \\
\text { sites }\end{array}$ & 1 & 5 & 4 & 1 & 6 \\
\hline Number of media articles posted & 2 & 4 & 5 & 1 & 4 \\
\hline \multirow{2}{*}{$\begin{array}{l}\text { Number of music/audio posts } \\
\text { Number of nage 'likes' }\end{array}$} & $\mathbf{0}$ & 3 & $\mathbf{0}$ & $\mathbf{0}$ & 5 \\
\hline & 3 & 3 & 6 & 2 & 4 \\
\hline $\begin{array}{l}\text { Number of page 'likes' } \\
\text { Number of people 'talking about' the } \\
\text { page }\end{array}$ & 2 & 4 & 6 & 2 & 5 \\
\hline $\begin{array}{l}\text { Number of photos posted of } \\
\text { employees/customers/applicants }\end{array}$ & 5 & 2 & 1 & 0 & 3 \\
\hline Number of posts by page & 2 & 4 & 5 & 5 & 4 \\
\hline $\begin{array}{l}\text { Number of posts containing } \\
\text { graphics/artwork }\end{array}$ & 1 & 7 & 2 & 2 & 6 \\
\hline
\end{tabular}




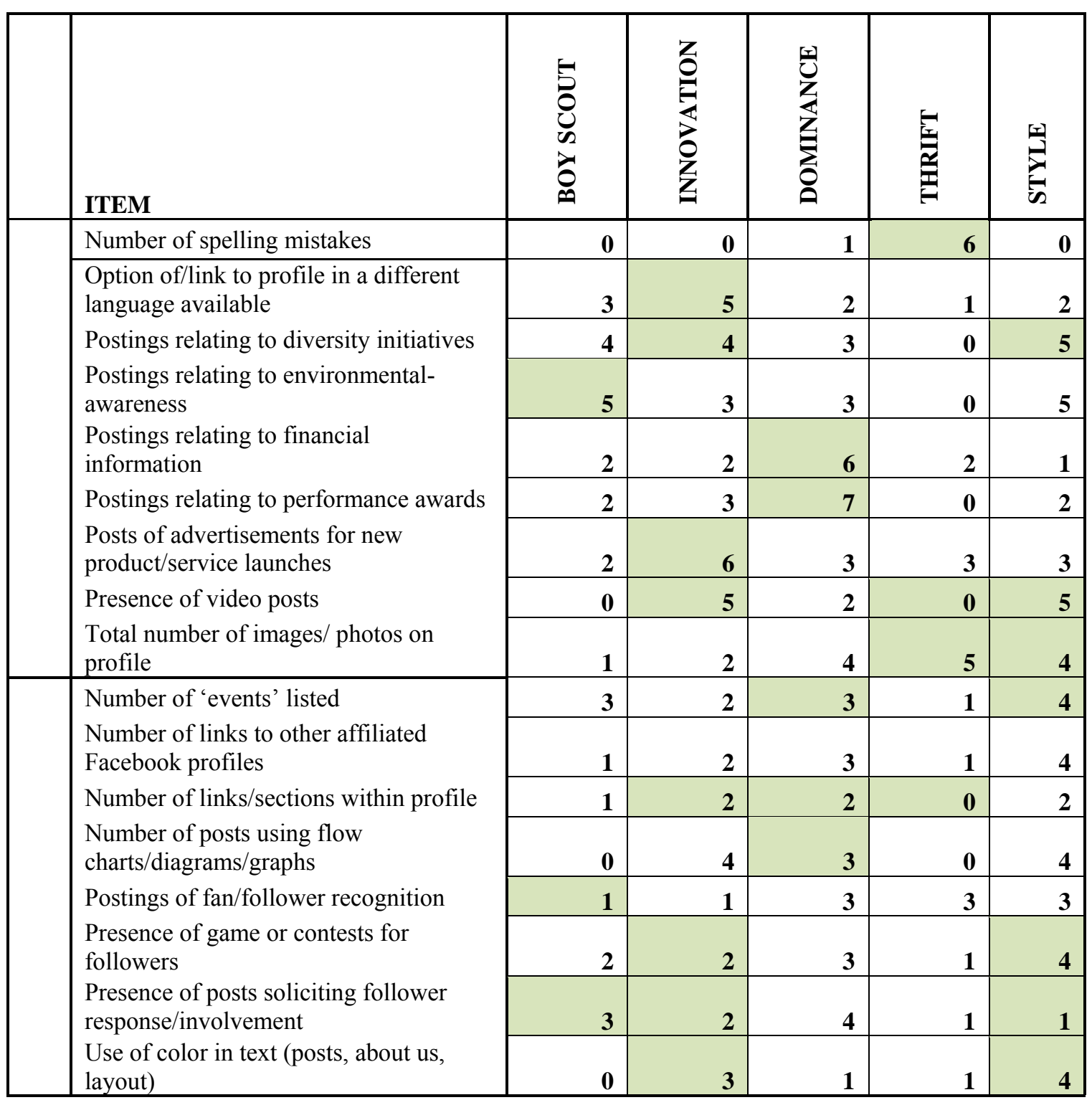

Note. Shaded cells represent original classifications based on RQ 1-5. Cell values represent total number of SMEs who chose that OP dimension out of total possible $\mathrm{N}=7$. 
Appendix K. Inter-class Correlation Coefficient (ICC) per Company $(N=102)$

\begin{tabular}{|c|c|c|c|c|c|}
\hline ID & Rank & Company & $\begin{array}{l}\text { Website } \\
\text { ICC }\end{array}$ & $\begin{array}{l}\text { Facebook } \\
\text { ICC }\end{array}$ & $\begin{array}{l}\text { No Exposure } \\
\text { ICC }\end{array}$ \\
\hline 1 & 500 & Molina Healthcare & .76 & .85 & \\
\hline 2 & 497 & Erie Insurance & .80 & .88 & \\
\hline 3 & 496 & Rockwell Collins & .78 & .92 & \\
\hline 4 & 495 & Smuckers & .90 & .67 & .83 \\
\hline 5 & 491 & $\begin{array}{l}\text { Alliant Techsystems } \\
\text { (ATK) }\end{array}$ & .62 & .91 & \\
\hline 6 & 490 & MetroPCS & .63 & .68 & .63 \\
\hline 7 & 489 & CIT Group & .87 & .74 & \\
\hline 8 & 485 & Charles Schwab & .88 & .72 & .81 \\
\hline 9 & 483 & Yahoo & .86 & .83 & .94 \\
\hline 10 & 482 & $\begin{array}{l}\text { Western \& Southern } \\
\text { Financial Group }\end{array}$ & .76 & .84 & \\
\hline 11 & 481 & Meritor & .77 & .84 & \\
\hline 12 & 480 & $\begin{array}{l}\text { SunGard Data } \\
\text { Systems }\end{array}$ & .84 & .68 & \\
\hline 13 & 478 & United Stationers & .89 & .85 & \\
\hline 14 & 475 & FMC Technologies & .72 & .92 & \\
\hline 15 & 474 & NetApp & .79 & .88 & .64 \\
\hline 16 & 473 & $\begin{array}{l}\text { Casey's General } \\
\text { Stores }\end{array}$ & .75 & .76 & \\
\hline 17 & 467 & Big Lots & .61 & .79 & .60 \\
\hline 18 & 466 & $\begin{array}{l}\text { Dick's Sporting } \\
\text { Goods }\end{array}$ & .90 & .95 & .91 \\
\hline 19 & 465 & Gannett & .81 & .78 & \\
\hline 20 & 464 & $\begin{array}{l}\text { Frontier } \\
\text { Communications }\end{array}$ & .69 & .54 & \\
\hline 21 & 461 & Pitney Bowes & .89 & .76 & \\
\hline 22 & 460 & Insight Enterprises & .77 & .63 & \\
\hline 23 & 459 & Con-way Freight & .67 & .75 & \\
\hline 24 & 458 & Harley-Davidson & .90 & .77 & .89 \\
\hline 25 & 456 & Clorox & .91 & .85 & .88 \\
\hline 26 & 454 & Owens Corning & .92 & .67 & \\
\hline 27 & 450 & $\begin{array}{l}\text { Live Nation } \\
\text { Entertainment }\end{array}$ & .73 & .88 & \\
\hline 28 & 447 & NCR & .84 & .84 & \\
\hline 29 & 445 & Western Union & .86 & .84 & .93 \\
\hline 30 & 444 & Kindred Healthcare & .94 & .86 & \\
\hline 31 & 442 & Avaya & .80 & .84 & \\
\hline 32 & 441 & Kelly Services & .88 & .84 & \\
\hline 33 & 440 & CH2M Hill & .84 & .92 & \\
\hline 34 & 439 & $\begin{array}{l}\text { Booz Allen } \\
\text { Hamilton }\end{array}$ & .84 & .92 & \\
\hline 35 & 438 & Spectra Energy & .67 & .88 & \\
\hline \multirow[t]{2}{*}{36} & 436 & Domtar & .64 & .76 & \\
\hline & & & 159 & & \\
\hline
\end{tabular}




\begin{tabular}{|c|c|c|c|c|c|}
\hline ID & Rank & Company & $\begin{array}{l}\text { Website } \\
\text { ICC }\end{array}$ & $\begin{array}{l}\text { Facebook } \\
\text { ICC }\end{array}$ & $\begin{array}{l}\text { No Exposure } \\
\text { ICC }\end{array}$ \\
\hline 37 & 435 & Foot Locker & .93 & .88 & .87 \\
\hline 38 & 434 & $\begin{array}{l}\text { Starwood Hotels \& } \\
\text { Resorts }\end{array}$ & .94 & .90 & .81 \\
\hline 39 & 431 & Ralph Lauren & .96 & .88 & .83 \\
\hline 40 & 430 & SanDisk & .89 & .95 & .75 \\
\hline 41 & 429 & $\begin{array}{l}\text { Auto-Owners } \\
\text { Insurance }\end{array}$ & .66 & .87 & \\
\hline 42 & 428 & Emcor Group & .85 & .79 & \\
\hline 43 & 424 & $\begin{array}{l}\text { O'Reilly } \\
\text { Automotive }\end{array}$ & .67 & .58 & .85 \\
\hline 44 & 422 & Exelis & .92 & .85 & \\
\hline 45 & 420 & Pacific Life & .89 & .82 & \\
\hline 46 & 417 & $\begin{array}{l}\text { Dr Pepper Snapple } \\
\text { Group }\end{array}$ & .96 & .88 & .89 \\
\hline 47 & 414 & Rubbermaid & .76 & .79 & .73 \\
\hline 48 & 410 & $\begin{array}{l}\text { Rockwell } \\
\text { Automation }\end{array}$ & .84 & .77 & \\
\hline 49 & 408 & Kodak & .89 & .89 & .80 \\
\hline 50 & 407 & Ryder & .83 & .81 & .89 \\
\hline 51 & 404 & Hershey's & .93 & .94 & .92 \\
\hline 52 & 400 & PetSmart & .91 & .89 & .78 \\
\hline 53 & 399 & Consol Energy & .67 & .89 & .92 \\
\hline 54 & 397 & Wesco International & .79 & .58 & \\
\hline 55 & 394 & iHeartRADIO & .94 & .95 & .92 \\
\hline 56 & 392 & Advance Auto Parts & .66 & .83 & .82 \\
\hline 57 & 391 & Symantec & .84 & .89 & .85 \\
\hline 58 & 388 & Mattel & .81 & .65 & .72 \\
\hline 59 & 387 & Precision Castparts & .82 & .68 & \\
\hline 60 & 385 & Amerigroup & .77 & .83 & .87 \\
\hline 61 & 383 & Dillard's & .89 & .87 & .85 \\
\hline 62 & 382 & $\begin{array}{l}\text { American Family } \\
\text { Insurance }\end{array}$ & .89 & .94 & \\
\hline 63 & 379 & Terex & .67 & .86 & \\
\hline 64 & 378 & $\begin{array}{l}\text { Advanced Micro } \\
\text { Devices (AMD) }\end{array}$ & .83 & .87 & \\
\hline 65 & 376 & $\begin{array}{l}\text { Sanmina } \\
\text { Corporation }\end{array}$ & .74 & .92 & \\
\hline 66 & 375 & $\begin{array}{l}\text { Agilent } \\
\text { Technologies }\end{array}$ & .84 & .91 & \\
\hline 67 & 373 & Dollar Tree & .79 & .69 & .60 \\
\hline 68 & 372 & Fifth Third Bancorp & .90 & .74 & \\
\hline 69 & 370 & MasterCard & .92 & .86 & .86 \\
\hline 70 & 368 & Celanese & .84 & .82 & \\
\hline 71 & 367 & Avery Dennison & .87 & .62 & \\
\hline 72 & 366 & $\begin{array}{l}\text { Cliffs Natural } \\
\text { Resources }\end{array}$ & .82 & .71 & \\
\hline 73 & 365 & Ecolab & .64 & .69 & \\
\hline \multirow[t]{2}{*}{74} & 363 & Winn-Dixie & .82 & .70 & .69 \\
\hline & & & 160 & & \\
\hline
\end{tabular}




\begin{tabular}{|c|c|c|c|c|c|}
\hline ID & Rank & Company & $\begin{array}{l}\text { Website } \\
\text { ICC }\end{array}$ & $\begin{array}{l}\text { Facebook } \\
\text { ICC }\end{array}$ & $\begin{array}{c}\text { No Exposure } \\
\text { ICC }\end{array}$ \\
\hline 75 & 360 & Barnes \& Noble & .91 & .93 & .86 \\
\hline 76 & 358 & $\begin{array}{l}\text { Interpublic Group } \\
\text { (IPG) }\end{array}$ & .74 & .68 & .91 \\
\hline 77 & 356 & $\begin{array}{l}\text { Alpha Natural } \\
\text { Resources }\end{array}$ & .69 & .88 & \\
\hline 78 & 354 & OfficeMax & .89 & .84 & .77 \\
\hline 79 & 351 & $\begin{array}{l}\text { Charter } \\
\text { Communications }\end{array}$ & .84 & .66 & \\
\hline 80 & 349 & Dole & .78 & .89 & .86 \\
\hline 81 & 346 & $\begin{array}{l}\text { Eastman Chemical } \\
\text { Company }\end{array}$ & .68 & .86 & \\
\hline 82 & 343 & Regions Bank & .86 & .89 & .63 \\
\hline 83 & 341 & Quest Diagnostics & .88 & .79 & .69 \\
\hline 84 & 340 & Ameren & .66 & .92 & \\
\hline 85 & 337 & Oshkosh B'gosh & .90 & .92 & .81 \\
\hline 86 & 335 & Boston Scientific & .85 & .90 & .60 \\
\hline 87 & 334 & Campbell Soup & .82 & .91 & .61 \\
\hline 88 & 332 & $\begin{array}{l}\text { Thrivent Financial } \\
\text { for Lutherans }\end{array}$ & .71 & .82 & \\
\hline 89 & 331 & $\begin{array}{l}\text { MGM Resorts } \\
\text { International }\end{array}$ & .93 & .93 & .76 \\
\hline 90 & 330 & Sonic Automotive & .71 & .91 & .79 \\
\hline 91 & 322 & $\begin{array}{l}\text { AECOM } \\
\text { Technology }\end{array}$ & .65 & .87 & \\
\hline 92 & 320 & AutoZone & .74 & .92 & .86 \\
\hline 93 & 318 & Grainger & .85 & .84 & \\
\hline 94 & 309 & Hertz & .79 & .70 & .79 \\
\hline 95 & 301 & Family Dollar & .80 & .85 & .69 \\
\hline 96 & 300 & Discover & .93 & .87 & .85 \\
\hline 97 & 299 & Ross Stores & .63 & .67 & .66 \\
\hline 98 & 295 & Principal Financial & .62 & .89 & \\
\hline 99 & 294 & Bed Bath \& Beyond & .91 & .94 & .76 \\
\hline 100 & 293 & Sherwin-Williams & .84 & .87 & .85 \\
\hline 101 & 292 & AGCO & .80 & .81 & \\
\hline 102 & 290 & Estee Lauder & .92 & .91 & .81 \\
\hline
\end{tabular}


Appendix L. Summary of Retained Final Objective Indicators of Image

\begin{tabular}{|c|c|c|}
\hline Dimension & Website & Facebook \\
\hline Boy Scout & $\begin{array}{l}\text { - frequency word 'support' } \\
\text { - frequency word 'safety' } \\
\text { - charity information listed } \\
\text { - employee recognition } \\
\text { - community involvement } \\
\text { - benefits listed }\end{array}$ & $\begin{array}{l}\text { - awards for best places to } \\
\text { work } \\
\text { - number of photos of people } \\
\text { - charity information posted } \\
\text { - frequency word 'respect' } \\
\text { posted } \\
\text { - community involvement } \\
\text { posts } \\
\text { - benefits listed } \\
\text { - continuing education } \\
\text { information } \\
\text { - frequency of word 'diversity' }\end{array}$ \\
\hline Innovation & $\begin{array}{l}\text { - frequency of word 'innovation' } \\
\text { - frequency of word 'technology' }\end{array}$ & $\begin{array}{l}\text { - frequency of word } \\
\text { 'innovation' } \\
\text { - frequency of word } \\
\text { 'technology' } \\
\text { - frequency of word 'risk' } \\
\text { - training/education } \\
\text { opportunities } \\
\text { - advertising new } \\
\text { product/service launches }\end{array}$ \\
\hline Dominance & $\begin{array}{l}\text { - frequency of word 'success' } \\
\text { - financial information provided } \\
\text { - awards for best places to work }\end{array}$ & $\begin{array}{l}\text { - awards for best places to } \\
\text { work } \\
\text { - performance awards listed } \\
\text { - financial information listed }\end{array}$ \\
\hline Thrift & $\begin{array}{l}\text { - frequency of word 'budget' } \\
\text { - amount of text in 'about us'* }\end{array}$ & $\begin{array}{l}\text { - amount images/photos* } \\
\text { - frequency of posts* }\end{array}$ \\
\hline Style & $\begin{array}{l}\text { - links to other social media } \\
\text { - environmental information listed }\end{array}$ & $\begin{array}{l}\text { - amount of graphics/art } \\
\text { - links to other social media }\end{array}$ \\
\hline
\end{tabular}

* indicates low levels or absence of that indicator 
VITA

ALEJANDRA C. MATAMALA

Born, Santiago, Chile

2001-2006

B.A., Psychology

George Mason University

Fairfax, Virginia

$2008-2012$

M.S., Industrial and Organizational Psychology

Florida International University

Miami, Florida

$2012-2014$

Ph.D., Industrial and Organizational Psychology

Florida International University

Miami, Florida

\section{PUBLICATIONS AND PRESENTATIONS}

Eaton, A. \& Matamala, A. (2014) Dangers of heteronormativity: The relationship between heteronormative beliefs and verbal sexual coercion. Archives of Sexual Behavior.

Matamala, A. C., Pace, V. L., \& Jacobs, H. (2010, April). Work engagement as a mediator between personality and citizenship behavior. Interactive poster session at the 25th Annual Conference of the Society for Industrial and Organizational Psychology, Atlanta, GA.

Matamala, A. C., \& Pace, V. L. (2012, August). Engagement as a Mediator between Personality and OCB: Measure Comparison. Poster presented at the 120th Annual Convention of the American Psychological Association, Orlando, FL.

Matamala, A., Sawhney, E., Drew, E., Thomas, J. \& Viswesvaran, C. (2012, August). Worthwhile or worthless? A meta-analysis of diversity training effectiveness. Paper presented at the annual meeting of the Academy of Management, Boston, MA.

Thomas, J., Kraus, E., Matamala, A., Michel, J., \& Viswesvaran, C. (2010, August). Conceptualizing global proactivity: A generalizable proactive process. Paper presented at the 70th Annual Meeting of the Academy of Management, Montreal, Canada. 